\title{
Foundations for Computable Topology
}

\author{
Paul Taylor
}

8 April 2009

\begin{abstract}
Foundations should be designed for the needs of mathematics and not vice versa. We propose a technique for doing this that exploits the correspondence between category theory and logic and is potentially applicable to several mathematical disciplines.

This method is applied to devising a new paradigm for general topology, called Abstract Stone Duality. We express the duality between algebra and geometry as an abstract monadic adjunction that we turn into a new type theory. To this we add an equation that is satisfied by the Sierpiński space, which plays a key role as the classifier for both open and closed subspaces.

In the resulting theory there is a duality between open and closed concepts. This captures many basic properties of compact and closed subspaces, despite the absence of any explicitly infinitary axiom. It offers dual results that link general topology to recursion theory.

The extensions and applications of ASD elsewhere that this paper survey include a purely recursive theory of elementary real analysis in which, unlike in previous approaches, the real closed interval $[0,1]$ in ASD is compact.
\end{abstract}

"In these days the angel of topology and the devil of abstract algebra fight for the soul of every individual discipline of mathematics."

(Hermann Weyl.)

"Point-set topology is a disease from which the human race will soon recover." (Henri Poincaré, quoted in Comic Sections by Des MacHale.)

"Logic is the hygiene that the mathematician practises to keep his ideas healthy and strong."

(Hermann Weyl, quoted in American Mathematical Monthly, volume 100, p 93.)

"Mathematics is the queen of the sciences and number theory is the queen of mathematics."

(Carl Friedrich Gauss)

"Such is the advantage of a well constructed language that its simplified notation often becomes the source of profound theories."

(Pierre-Simon Laplace, quoted in Mathematical Maxims and Minims by N. Rose, Raleigh Rome Press, 1988.)

Contents

1 Foundations for mathematics Category theory and type theory

Method and critique

Stone duality

Always topologize

The monadic framework

\begin{tabular}{|r|rl}
\hline 2 & 7 & The Sierpiński space \\
\hline 5 & 8 & Topology using the Phoa principle \\
\hline$\overline{9}$ & 9 & Discrete mathematics \\
\hline 13 & 10 & Underlying sets \\
\hline \hline 16 & 11 & Scott continuity \\
\hline \hline 20 & 12 & Beyond local compactness \\
\hline
\end{tabular}




\section{Foundations for mathematics}

1.1. To mis-quote Gauss and Eric Bell, Logic is the Queen and Servant of Mathematics. Ever since Logic became a mathematical discipline in the nineteenth century, foundational discussions (whether using sets, types or categories) have been based on four premises:

(a) logicians know best, and mathematicians should be grateful for what they are given;

(b) in particular, it is up to mathematicians to glue the continuum back together from the dust that logicians have provided; whilst

(c) they have convinced each other that foundations should permit arbitrary abstraction of mathematical processes; and

(d) anyone who tinkers with the foundations risks bringing the whole edifice of science crashing down.

1.2. The first two of these attitudes flew in the face of the tradition of the preceding two millennia, in which, for example, Euclid proved lots of rigorous theorems about lines, circles and other figures as things in themselves. Frege and Hilbert believed the third, and were admonished for it by Russell and Gödel. We now have a compromise situation in which the admissible forms of abstraction are in equilibrium with the extent to which the typical mathematician understands them. The result of this is that we debate the "truth" of the axiom of choice, etc., without any point of reference in the real world against which to measure it. It is impossible to axiomatise arbitrary abstraction, and nor is the status quo clearly defined.

There is, however, a natural baseline for foundations, namely computation (also called recursion), because the Church-Turing thesis says that all forms of computability are essentially equivalent. Mathematics in the time of Gauss and before was computational and, as we shall see, this also agrees with many of the intuitions of modern general topology. However, we also need to consider logically stronger systems, in particular in order to study the behaviour of computation and topology, for example whether a program terminates or an open subset covers a compact one.

1.3. What do we mean by "foundations" in mathematics anyway?

The basics are counting and measurement. Over the millennia, mathematicians have found clever ways of deriving results about these things by means of inventions whose originally fictitious nature is still remembered in their names, such as irrational, imaginary and ideal numbers.

For example, the front-line issue in mathematics during the 16 th century was how to solve cubic equations. Scipione del Ferro and Niccolò Tartaglia had already found a method for the case where there is only one (real) root. Rafaele Bombelli showed how to extend this method to the case where there are three real roots, but his intermediate calculations involved square roots of -1 , which had not been needed in the original case [FG87, §8.A].

The foundational question here is not so much how to "define" $\sqrt{-1}$ as to show that, if we introduce it in the middle of a calculation but then eliminate it, we will not obtain a contradiction. More precisely, whatever we do get by this method could have been found using real numbers alone. In logical jargon, complex numbers are a conservative extension.

So what is their value? Just try doing Fourier analysis without them, and see what a mess you get into!

An axiom was originally a statement that is obvious and needs no justification, but the meaning of this word has changed. It is still a starting point, but one that is carefully chosen to facilitate the development of a particular body of abstract theory. The purpose of foundations should be reinterpreted in the same way, on a larger scale. 
1.4. As the quotation from Laplace says, language and notation can be powerful driving forces behind a mathematical theory, whilst a bad notation (such as using the letters CDILMvx to write numbers) obstructs even the simplest task. As the subject has got more powerful and sophisticated, so the design of language and notation has become a professional discipline in itself. This discipline is, or at least ought to be, called Logic. It has now enjoyed well over a century of mathematical development and applications.

However, this does not justify the prescriptive role that Logicists and their successors claimed, because Mathematics has also advanced during the same time. In particular, the conceptual structure of the subject (such as Emmy Noether's Modern Algebra) has developed independently of its alleged foundations in set theory.

The philosophical thesis of this paper is that we can make Logic the servant of (a particular discipline in) Mathematics. Starting from what we see as the principal theorems of the Mathematics that we want to do, we employ certain theorems that link categorical and symbolic Logic as tools to create a new language for our chosen Mathematical discipline. The formal method that we propose is set out in the next two sections.

In motivating this, we shall use the metaphor that a logician is like an engineer or an architect who has been commissioned to design a new gadget or building for a client, who in turn has customers. However, we stress that our proposal is not just philosophy, but founded on extensive technical development in a number of mathematical disciplines.

Being a mathematician, I only discuss this thesis very briefly, leaving philosophers to explore it more fully. I feel compelled to say some of these things because I have found it impossible to do a substantial piece of research on the reformulation of general topology within the "orthodox" framework that my colleagues, referees, conferences, journals, universities and funding agencies say that I should use.

1.5. The instruments that we shall apply to designing a foundation for (parts of) mathematics are category theory and modern symbolic logic, i.e. proof theory and type theory. Many excellent Mathematicians "work in an elementary topos" or "in Martin-Löf type theory", but if we were to do this we would once again be bowing to Logicians as our masters. So, in order to employ these disciplines as servants of mathematics, we make our own selection from the menu of techniques that they offer.

Category theory grew out of (algebraic) topology and algebraic geometry, without any foundational pretensions in the beginning ML88, McL90. It is now used throughout mathematics, primarily for the notion of universal property or adjunction. Mathematicians in other fields who have any feel for the unity of the subject express the headline theorems of their speciality in this form. The categorical approach seems to be very effective in taking decades' worth of experience in one discipline, distilling it into adjunctions and a very small number of other abstract but widely applicable concepts, and transferring it to other subjects. These now include the theoretical parts of computer science and physics. Universal properties will provide the client's brief to the architect of the new foundations.

On the other hand, symbols are the currency of everyday business in mathematics, so they must be the way in which the new foundations will be used. However, any pre-existing symbolic language acquiesces to a lot of foundational prejudgements without giving them proper scrutiny. We aim to replace these assumptions with our own principles. These are, in the first instance, formulated using category theory, because it is agnostic.

We shall demonstrate the inter-relationship between category theory and symbolic logic in the next section. It is this that makes them together a powerful method for devising new language and notation for mathematics. 
1.6. The greater part of this paper is a survey of a research programme (ASD) that applies the proposed methodology in the case where the client is general topology, and its customers include geometric topologists, analysts and theoretical computer scientists.

The new theory axiomatises continuity directly, without any recourse to set theory or its usual alternatives. It begins from ideas concerning the duality of algebra and topology that were introduced by Marshall Stone $(\$ \$ 4,5 \& 77)$. These are put in an abstract form (hence the name ASD) using a monadic adjunction (\$6), along with an algebraic equation that characterises the way in which the Sierpiński space uniquely classifies open subspaces (\$8). Surprisingly much of the basic theory of open, closed, compact and Hausdorff spaces and subspaces can be recovered in this setting, and the resulting theory is computable, at least in principle.

However, when we try to apply this theory to discrete mathematics and computation, we find that we need something to play the role of "sets". For this, we those spaces that come with $\exists$ and $=$, which we call overt discrete ( 9 ). They can be made to behave like traditional set theory if we add the non-computable hypothesis that all spaces have "underlying sets" (\$10).

The infinitary joins in general topology make a surprisingly late explicit appearance, completing the re-axiomatisation of locally compact spaces $(\$ 11)$ and providing the basis for recursive elementary real analysis. To go beyond that, however, we need to re-think the underlying categorical framework $(12$, which is work in progress.

The purpose of this paper is to tell the whole story of ASD, from beginning to end, and it summarises work that would fill a book. This means that it has to take a "broad brush" approach, and it is not encyclopedic. If you are looking for the details of a particular topic, you should therefore consult the articles that are cited here.

Since many of the details of the theory are still missing, the time is not ripe to write a textbook about ASD. A few arguments are given here in full if they are foundationally important but presented inadequately in the other papers. In particular, we give the whole of the proof that these methods characterise an elementary topos without mentioning subobjects (\$9.3).

1.7. The most important mathematical achievement of the ASD programme so far is an account of recursive elementary real analysis that includes the Heine-Borel theorem. Recall that this says that $[0,1] \subset \mathbb{R}$ is compact in the sense that every cover by a family of open subspaces contains a finite sub-family that also covers [I].

This is the practical reason why no computable foundations have previously been developed that are adequate for mathematics. If we take ordinary set theory and analysis on the one hand, and computability theory on the other, putting them together in the obvious way, that is, by defining $\mathbb{R}$ as the set of computably representable real numbers, we find that this important result fails. Roughly speaking, this is because we may list the computable real numbers $a_{0}, a_{1}, \ldots$ according to the codes that represent them, and cover each of them by intervals of length $\frac{1}{4}, \frac{1}{8}, \ldots$, no finite subset of which would suffice. See [BR87, §3.4] for a very clear account of this.

The applications of Abstract Stone Duality to elementary real analysis in [J] also demonstrate the utility of the new language. That paper provides an entirely different introduction to ASD that is aimed at a general mathematical audience - it assumes only first year analysis and a facility with formal systems, completely avoiding the underlying category theory. It is complementary to the account in this paper, neither of them assuming familiarity with the other.

Although we mention computable foundations, we actually say very little about practical implementation here. This is a very interesting topic in itself, particularly in relation to elementary analysis, so more will be said about it in future work [Bau08, K]. 


\section{Category theory and type theory}

2.1. Gerhard Gentzen, the father of proof theory, set out the way in which we reason with the logical connectives $(\wedge, \vee, \Rightarrow)$ and quantifiers $(\forall$ and $\exists)$ Gen35.

Consider, for example, how we use and prove implications $(X \Rightarrow Y)$. If we know both $X$ and $X \Rightarrow Y$ then we also know $Y$; this is traditionally called modus ponens. More generally, we may have proofs $\nabla_{1}$ and $\nabla_{2}$ of $X$ and $X \Rightarrow Y$ respectively, from a set $\Gamma$ of assumptions. Putting these together gives a proof of $Y$, as illustrated on the left:
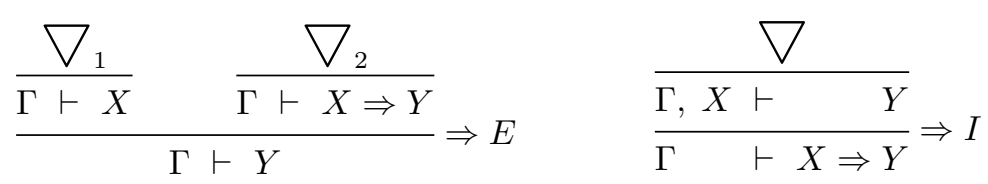

Conversely, how can we prove the implication $X \Rightarrow Y$ ? By definition, it is by giving a proof $\nabla$ of $Y$ that may assume $X$, as on the right. We need to maintain a list $\Gamma$ of such assumptions in order to keep track of the extra ones that we add and discharge in proofs of implications; $\Gamma$ is called the context.

These two patterns are called the elimination and introduction rules for $\Rightarrow$ (hence the labels $\Rightarrow E$ and $\Rightarrow I)$, because of the disappearance or appearance of this symbol in the conclusion. There are similar rules for the other connectives.

2.2. Under Haskell Curry's analogy with types How 80 , Sel02, their connectives $(\times,+, \rightarrow)$ and quantifiers $(\Pi$ and $\Sigma$ ) were later expressed in a similar way. But now, in place of the bald assertion that $X \Rightarrow Y$, we now have a function $X \rightarrow Y$ that takes values of one type to those of the other. This function needs a name. Bertrand Russell [RW13, *20] originally called it $\hat{x}(\phi x)$, but the hat evolved into a lambda:

$$
\frac{\Gamma \vdash a: X \quad \Gamma \vdash f: X \rightarrow Y}{\Gamma \vdash f a: Y} \rightarrow E \quad \frac{\Gamma, x: X \vdash \quad p \quad: Y}{\Gamma \quad \vdash \lambda \cdot p: X \rightarrow Y} \rightarrow I
$$

Since types come with terms, these too need rules for their manipulation. The beta rule arises from introducing and then eliminating the symbol, and the eta rule from the converse,

$$
\frac{\Gamma \vdash a: X \quad \Gamma, x: X \vdash p: Y}{\Gamma \vdash(\lambda x \cdot p) a=[a / x]^{*} p: Y} \rightarrow \beta \quad \frac{\Gamma \vdash f: X \rightarrow Y}{\Gamma \vdash f=\lambda x \cdot f x: Y} \rightarrow \eta
$$

where $[a / x]^{*} p$ indicates substitution $(2.7)$. Now the context $\Gamma$ must include both assumptions and typed variables or parameters.

2.3. If we have such a system of rules for the connectives that we use — instead of encoding them in a much more general and powerful all-encompassing foundational monolith — then we have some chance of computing in our system. Of course, mathematicians always did that, before logicians came along: the difference is that the system is now much bigger (\$1.4), and so requires a more formal relationship amongst its parts, and amongst the specialists in very diverse disciplines $(\$ 3.6$.

In the simply typed $\lambda$-calculus, for example, we merely have to keep applying the $\beta$-rule, and then we are guaranteed to get to the normal form ... eventually. In order to prove termination of this process we use induction on the complexity of the types that are involved. For uniqueness, we need to know that, whenever we have a choice of redexes (reducible expressions), the two results can be brought back together by further reductions CR36. Both of these results depend in delicate ways on the formulation of the system. 
Whilst it may be rather naïve to rely on this "guarantee" in practice, we can hand the formal system over to the experts in manipulating symbols. They do not need to understand their original meaning, but may use their own experience of similar systems to make the computation more efficient. Even so, the mathematical axioms and not the operations of a machine determine the fundamental rules. This has been done very successfully in functional programming, which is now almost as fast as machine-oriented approaches Hud89, HHPJW07.

There is, in fact, much profit to be made from collaborating with computational specialists only via a formal language. They may be able to transform it into something entirely different, that works qualitatively faster than the methods that the mathematician originally had in mind. One example of this that is indirectly related to the ideas in this paper ( 8.4 ) is the continuation-passing method of compiling programs, because of its similarity to the way in which microprocessors handle sub-routines App92.

Another arises in the solution of equations involving real numbers. Constructive analysis discusses interval-halving methods [J, §1], but if we were to compute with them verbatim we would only get one new bit per iteration, whilst Newton's algorithm doubles the number of bits each time. But the verbatim reading is a mis-communication - we only halve the interval for the purpose of explanation. A practical implementation may choose the division in some better place, based on other information from the problem. Indeed, this what is done in logic programming with constraints Cle87. Putting the ideas together, we can turn one algorithm into the other [Bau08, K].

Altogether, we can think mathematically in one way, but obtain computational results in another.

2.4. Bill Lawvere Law69 recognised the situation in type theory $(\$ 2.2$ as a special case of that studied by categorists. We say that the functors $\mathrm{F}: \mathcal{S} \rightarrow \mathcal{A}$ and $\mathrm{U}: \mathcal{A} \rightarrow \mathcal{S}$ between categories $\mathcal{A}$ and $\mathcal{S}$ are adjoint $(\mathrm{F} \dashv \mathrm{U})$ if there is a bijection (called transposition)

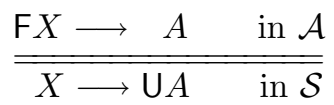

that is natural in $X$ and $A$, which means that it respects pre- and post- composition with morphisms in the two categories. The transposes of the identities on $\mathrm{F} X$ and $\mathrm{U} A$ are called the unit and counit of the adjunction, respectively,

$$
\eta_{X}: X \rightarrow \mathrm{U}(\mathrm{F} X) \quad \text { and } \quad \varepsilon_{A}: \mathrm{F}(\mathrm{U} A) \rightarrow A,
$$

and these satisfy equations known as the triangle laws:

$$
\mathrm{U} \varepsilon_{A} \cdot \eta_{\mathrm{U} A}=\mathrm{id}_{\mathrm{U} A} \quad \text { and } \quad \varepsilon_{\mathrm{FX}} \cdot \mathrm{F} \eta_{X}=\mathrm{id}_{\mathrm{F} X}
$$

The use of the letter $\eta$ for different things in category theory and type theory is most unfortunate, but it is a historical accident that is very well established in both subjects.

2.5. For example, the correspondence

$$
\frac{\Gamma, x: X \vdash p: \quad Y}{\overline{\Gamma \quad \vdash x \cdot p: X \rightarrow Y}} \quad \text { or } \quad \frac{\Gamma \times X \longrightarrow Y}{\Gamma \longrightarrow Y^{X}}
$$

is bijective on the left by the symbolic rules $(2.2)$, and on the right as the adjunction $(-) \times$ $X \dashv(-)^{X}$. However, purely categorical textbooks cover many pages with diagrams expressing arguments that could be written much more briefly by adopting the notation $\lambda x . p$ instead of $\tilde{p}$ (cf. 6.1). 
This analogy can be followed systematically through all of the logical connectives:

(a) Where there is a symbolic rule like $\lambda$-abstraction that binds variables or changes the context, it corresponds to the adjoint transposition; case analysis, recursive definitions and pairing are also examples of transposition.

(b) The "algebraic" operation ev, $\pi_{0}$ or $\pi_{1}$ that does not change the context is provided by the co-unit $\varepsilon$ of the adjunction in the case of $\rightarrow$ and $\times$, but the unit $\eta$ gives the inclusions $\nu_{0}$ and $\nu_{1}$ for + .

(c) The triangle laws provide the $\beta$ - and $\eta$-rules.

(d) One of the naturality conditions for the adjunction defines the effect of the construction on morphisms. The other says that we may substitute within $\langle-,-\rangle$ and $\lambda$, but in the case of $\vee$ and $\exists$, it gives the so-called commuting conversions or continuation rules.

(e) Substitution under $\vee$ and $\exists$ must be stated categorically by means of additional conditions $(\$ 2.9)$.

The reason why some of the connectives $(\Rightarrow, \wedge, \rightarrow, \times)$ work one way round and the others $(\vee$, $\exists,+$ ) differently is that they are respectively right and left adjoints to something that is simpler. This is described more fully in Tay99, §7.2].

2.6. In order to formalise this relationship between adjunctions and systems of introduction, elimination, $\beta$ - and $\eta$-rules, we need a fluent translation between diagrammatic and symbolic languages. In fact, we just require a way of turning a type theory that has all of the structural rules (identity, cut, weakening, contraction and exchange, in proof-theoretic terminology) into a category with finite products. Then we shall be able to ask when this category obeys other universal properties, and read off from them the corresponding symbolic rules.

The following method also works for dependent types, i.e. possibly containing parameters, but we shall avoid them in this paper. There are, conversely, ways of defining a language from a pre-existing category Tay99, §7.6], but these are more complicated and will not be needed here. Systems such as linear logic that do not obey all of the structural rules correspond to different categorical structures. These might, for example, be tensor products $(\otimes)$, which categorists understood long before they did predicate logic [ML63. It is therefore often the syntax that requires the most innovation, for example using Jean-Yves Girard's proof nets Gir87.

A type theory $\mathcal{L}$ of the kind that we shall consider has recursive rules to define

(a) types $(X)$;

(b) contexts $(\Gamma)$, which are lists of typed variables (parameters) and equations between terms, understood as hypotheses;

(c) terms $(\Gamma \vdash a: X)$ of each type $X$, whose free variables belong to the given context $\Gamma$; and

(d) equations $(\Gamma \vdash a=b: X)$ between these terms.

2.7. We have already used the substitution operation $[a / x]^{*} c$, which must be defined carefully in order to avoid variable capture by $\lambda$-abstraction. Another operation (called $\hat{x}^{*} c$ in [Tay99, §1.1] but not given a name elsewhere) weakens $c$ by pretending that it involves the actually absent variable $x$. These satisfy the extended substitution lemma,

$$
\begin{array}{lrl}
{[a / x]^{*}[b / y]^{*} c=\left[[a / x]^{*} b / y\right]^{*}[a / x]^{*} c} & {[x / y]^{*} \hat{x}^{*}[y / x]^{*} \hat{y}^{*} c=c} \\
{[a / x]^{*} \hat{x}^{*} c=c} & {[a / x]^{*} \hat{y}^{*} c=\hat{y}^{*}[a / x]^{*} c} & \hat{x}^{*} \hat{y}^{*} c=\hat{y}^{*} \hat{x}^{*} c
\end{array}
$$

where $x \not \equiv y, x, y \notin \mathrm{FV}(a)$ and $y \notin \mathrm{FV}(b)$. 
2.8. Like so many other operations in mathematics, substitution is defined by its action on other objects. So, when we abstract the operation from its action, it has an associative law of composition, which brings it into the realm of category theory.

The objects of the category are, abstractly, the contexts $\Gamma$ of the type theory. More concretely, $\Gamma$ is represented by the set of terms that are definable using free variables chosen from $\Gamma$. The substitution and weakening operations define morphisms

$$
\begin{aligned}
{[a / x]^{*} c \longleftrightarrow c \quad \hat{x}^{*} d \longleftrightarrow } & \\
\Gamma & \longrightarrow[a / x] \\
\Gamma & \longrightarrow \Gamma, x: X] \stackrel{\hat{x}}{\longrightarrow} \Gamma .
\end{aligned}
$$

It is convenient to draw the arrows in such a way that substitution looks like inverse image, which is why we write it with a star. We also draw weakening with a special arrowhead and call it a display map. The five equations of the extended substitution lemma say that certain diagrams formed from these arrows commute, i.e. segments of paths of arrows that match one pattern may be replaced by another. For example, the composite of the two arrows above is the identity.

Altogether, we have defined, not the category itself, but an elementary sketch. That is, whilst we have spelt out all of the objects, we have only given a generating set of arrows. The category $\mathrm{Cn}_{\mathcal{L}}^{\times}$itself consists of all paths formed from these arrows, subject to the equations of the given symbolic theory $\mathcal{L}$ and those of the extended substitution lemma. There is a normalisation theorem that says that any morphism $\Gamma \rightarrow \Delta$ is essentially an assignment to each variable in $\Delta$ of a term of the same type, but formed using variables from the context $\Gamma$. In keeping with the traditional names of categories ("of objects X and morphisms Y"), I therefore called this the category of contexts and substitutions. The details of this construction are given in Tay99: see $\S 4.3$ for the simply typed case and Chapter VIII for its generalisation to dependent types.

The category $\mathrm{Cn}_{\mathcal{L}}^{\times}$has the universal property that interpretations of the theory $\mathcal{L}$ in another category $\mathcal{T}$ correspond bijectively (up to unique isomorphism) to functors $\mathrm{Cn}_{\mathcal{L}}^{\times} \rightarrow \mathcal{T}$ that preserve the relevant structure. This means that it is the classifying category for models of this structure.

Type theories are presented in a recursive fashion, whilst categories have an associative law of composition, but anyone who has ever written any functional programs knows that associativity and recursive definitions do not readily mix. Andrew Pitts defined the classifying category in this way in [Pit00, §6]; it was the complexity of this approach that led me to the construction via a sketch. It demarcates the things that are naturally described in a recursive and symbolic way from those that are best done associatively and diagrammatically.

2.9. Many of the comparisons and contrasts between diagrammatic and symbolic presentations are illustrated by Lawvere's notion of hyperdoctrine Law69. This represents the quantifiers $\Pi$ and $\Sigma$ as the right and left adjoints $\left(c f . \$ 2.5\right.$ to the substitution functors $f^{*}: \mathcal{A}[Y] \rightarrow \mathcal{A}[X]$ that are defined by a map $f: X \rightarrow Y$. However, induced and restricted modules along a ring homomorphism also provide adjunctions like these. The difference is that, in symbolic logic, we may substitute for parameters within the scope of the quantifiers. This is not part of the correspondence in $\$ 2.5$, so category theory must express it by another axiom, known as the BeckChevalley condition, which does not hold for general ring homomorphisms. Another property that is peculiar to the existential quantifier is the equation $\sigma \wedge \exists x . \phi x \Leftrightarrow \exists x . \sigma \wedge \phi x$, which Lawvere called the Frobenius law.

So category theory is

(a) agnostic in treating both modules and quantifiers in a general way;

(b) more precise in identifying the substitution operations in the former that go unremarked in symbolic logic; and

(c) more efficient than giving pages of proof rules like those in $\S ₫ 2.1$ for specifying which structures we want to include in our system. 
On the other hand, it is

(d) clumsy and very difficult for the student to learn if we are actually just interested using in the quantifiers to express other mathematics.

2.10. In requiring this structure for all maps $f: X \rightarrow Y$, hyperdoctrines have a hidden bias towards set theory. In order to apply Lawvere's ideas to topology or domain theory (for example, to model second-order quantification over type variables), we must restrict the subclass of maps that admit quantification. It is, in fact, more accurate to say that quantification is adjoint to weakening Tay99, Ch. IX].

Set theory will be important in this paper, not as a competing logical theory, but as an analogous mathematical one, providing another example of Stone duality (4.5). We aim to study topology, which is about continuous things, so by "set theory" we shall mean any treatment of discrete objects, which may be in an elementary topos, using type theory or in some axiomatisation of the $\in$-relation.

The key difference that will emerge is that in the logic of discrete things we may use quantifiers ranging over any object, whilst in topology only some spaces may play this role. An object $X$ that may serve as the range of a universal quantifier is called compact. More generally, a map that admits parametric universal quantification $\grave{a}$ la Lawvere is called proper, and we think of the map as a continuous family of compact spaces. Similarly, a map with existential quantification is open, but we need to introduce a new word, overt, for the corresponding objects.

2.11. Nevertheless, Lawvere's insight is the key to our method, when we turn it around. Having seen many systems of rules and adjunctions for different logical connectives, we may recognise the general pattern and apply it to mathematics.

Many theorems in mathematics (our client's brief) may be formaluted as adjunctions, to which we apply Lawvere's correspondence in reverse: we invent new symbols whose introduction, elimination, abstraction, beta and eta rules exactly capture the adjunctions. We shall do this in 86.3 for sobriety, in $\S \varangle 6.6$ for $\Sigma$-split subspaces, in $\$ 9.11$ for definition by description, in $\$ 10.6$ for underlying sets, in \$11.11 for Dedekind completeness and in $\$ 12.3$ for more general subspaces.

2.12. The new symbols are not meant to be merely abbreviations for common lemmas about the old structure, but to stand on their own feet as principles of reasoning in the new one. As with complex numbers $(\$ 1.3)$, we want to be able to compute with them without worrying about what they mean $(\$ 2.3)$. This means that we need to ensure that they are accompanied by a complete set of rules of inference.

One good indicator of sufficiency is being able to prove a normalisation theorem. Typically, this says that say that any term that makes arbitrary use of the introduction and elimination rules is provably equivalent to one that uses

(a) the new elimination rule only for free variables of the new type; and

(b) the new introduction rule only on the outside of the term.

The equivalence between the categorical and symbolic constructions is deduced from theorems of this kind.

\section{Method and critique}

We argued in 1.5 that category theory is adept at capturing the important ideas in a mathematical discipline in the form of universal properties, whilst axioms may be expressed in symbolic logic as systems of introduction and elimination rules. Then in $\$ 2$ we demonstrated the formal connection between these. 
3.1. On this basis, we therefore propose a methodology for devising a new foundational system for a mathematical discipline. This is summed up by the diagram

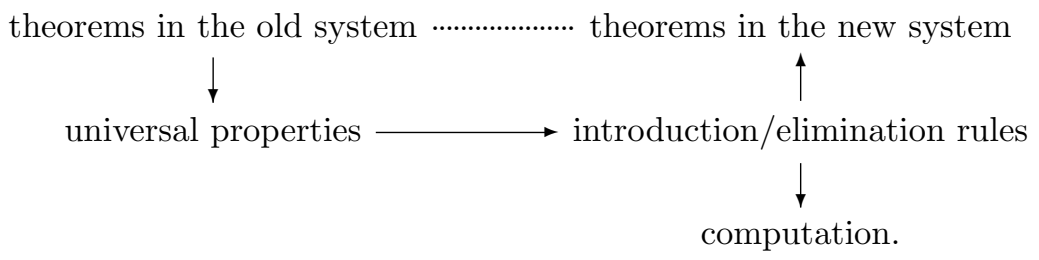

In this, the task of the journeyman mathematician, i.e. the deduction of theorems from axioms, is to fill in the upward arrow on the right. Hopefully, this will make the rectangle "commute" in the sense that we recover the old theorems from the new axioms.

However, this job only makes sense in a professional framework in which the axioms have been chosen to capture the intuitions and applications of the discipline. This choice is represented by the downward arrow on the left.

The constructions that we sketched in $\S \S 2.8$ provide the horizontal arrow in the middle, whilst the computational interpretation (bottom right) depends on the details of the resulting calculus (\$2.3).

We shall show how this programme works for the example of general topology in the remaining sections of this paper, with some hints about how to apply the ideas to other subjects.

3.2. The first arrow in some sense derives the axioms from the theorems.

Euclid and Bourbaki gave us a style of presenting mathematics that begins from axioms which (so far as the text is concerned) come out of the blue, and deduces theorems from them as if the results were obvious and inevitable. Abel described Gauss as "like a fox, who effaces his tracks in the sand with his tail". As a result, lesser mathematics think that it is enough to state a bunch of axioms in order to justify the relevance of their results, whilst students learn nothing about the ways in which their masters discovered their theorems.

In following our methodology, we cannot "write down all the axioms first", because finding the right axioms is the objective of the investigation. Being able to deduce the old theorems from the new axioms is an experimental test of the axioms that we propose. So before we have discovered the final version of the axiom system, we need to conduct preliminary experiments in ad hoc frameworks ( $c f . \$ 5)$.

Along the way, like any engineer, we shall assemble several prototypes. Of course this happens all the time in mathematics too Lak63 — it is just the textbooks that falsify history by saying otherwise. At the end of the day, we want to state our chosen axioms and deduce the important theorems from them. But that will indeed be the end of the process, which will then no longer be an active piece of foundational research.

For a modern study of a wide range of techniques for discovering the principles of mathematical disciplines, see [Cor03].

3.3. Counterexamples, of course, play an major part in the empirical development of mathematics. Unfortunately, they are sometimes given such a degree of prominence that they can stifle subsequent progress.

Typically, the argument behind a counterexample may prove

$$
D, \quad E, \quad F \quad \neg \quad \neg G,
$$

entirely rigorously. Then we are expected to agree that,

therefore, $E$ is false. 
Why? $D, F$ and $G$ had exactly the same status in the proof, so why pick on $E$ ? This is an argument by authority, not a valid piece of mathematics: the author has simply used sleight-ofhand to make us accept his prejudices. These become embedded in the literature, and treated as theorems, on which whole theories are built. Having forgotten that the counterexample depended on cultural assumptions, people then resist the introduction of a new paradigm.

This abuse of counterexamples is very common, but surely the one that has caused the greatest intellectual paralysis is Cantor's, that no set is in bijection with its powerset. On this he built his theory of cardinality, which is far too coarse to be of any use in mainstream mathematics. It nevertheless says that a set $X$ can only satisfy $X \cong X^{X}$ in the trivial cases $X \equiv \emptyset$ or $\mathbf{1}$. Apparently, the untyped $\lambda$-calculus is therefore inconsistent. Dana Scott eventually saw through this, and constructed topological lattices with this property Sco93. As these can be embedded in presheaf toposes, we see that Cantor's supposedly fundamental theorem of set theory actually relies on excluded middle.

The message that we ought to take from a counterexample is no more than that you can't do it like that. Maybe someone someday will think of a different way. They will identify underlying assumptions $A, B$ and $C$ that had previously been overlooked, and achieve all of $D, E, F$ and $G$ on some other basis, using $A^{\prime}, B^{\prime}$ and $C^{\prime}$ instead.

In particular, just as in the use of formal methods for security (putting a strong lock on a weak door), it is the very rigour of a fragment of argument that is most misleading about the bigger picture. In our architectural metaphor, counterexamples are reasons for choosing one general scheme over another, whilst theorems hold the building up once we have chosen which plan to build. A tower block may later turn out to be a mistake for sociological reasons that have nothing to do with how accurately its plans were drawn.

It would, perhaps, be useful to make a clear distinction between the main track of a theory (from axioms to theorems) and its peri-theory. This is the discussion around the theory, consisting of the important examples, prototypes and counterexamples that led us to the choice of statements of the axioms and theorems. Other examples of peritheory are converses that lead back from the main theorem to (the necessity of) the chosen axioms, and more generally the lists of equivalent alternative formulations of the axioms that some textbooks like to give.

3.4. Unfortunately, it is not as straightforward as we have suggested to work from a client's brief: this cannot be taken literally, and has to be negotiated.

If the client is allowed to dictate what is fundamental, the new foundations will be no more flexible than the old ones. It takes an outsider's perspective to distinguish the key elements of an enterprise from the accumulation of detail. The first arrow does not therefore just copy the theorems and chapter headings of the old theory.

In approaching a mathematical discipline, category theory often focuses on ideas that its specialists previously regarded as trivial. For example, the subobject classifier and Sierpiński space $(\$ 7)$ are merely two-element sets in classical set theory and topology. Also, mathematicians have acknowledged that the theorems that create important structures are adjoints — but an adjunction is a two-sided relationship, in which the other partner typically does something that appears to be mere bureaucracy, namely to "forget" the same structure.

3.5. Another issue on which the clients' preconceptions need to be challenged is the identification of their most valuable products. It is up to their customers to do this. For example, set theorists put much of their effort into considering infinite cardinals, but the demand from the market is for more mundane things, such as quotients of equivalence relations, which are axiomatised explicitly in categorical logic $(\$ 9.1(\mathrm{e})$.

On the other hand, maybe led by an over-emphasised counterexample, specialists sometimes develop variant forms of a subject that omit properties which the customers may regard as essential. One case of this is compactness of the interval $[0,1] \subset \mathbb{R}(\$ 1.7)$, which is absent from Bishop's 
theory BB85.

3.6. Negotiation of this kind is how we answer the final objection to changing foundations, namely that the prevailing set-up is crucial for the whole of science $(1.1(\mathrm{~d}))$.

Science and mathematics are systems whose component disciplines interact across an interface. The higher-level components depend only weakly on the details of those below, but do have requirements for certain features. This is a commonplace in modern technology. It is also well known in the wider picture of science: whilst chemistry depends on physics, it only uses four sub-atomic particles, organic chemistry for the most part only four elements, and genetics only four bases and twenty amino acids. Physics very probably relies on compactness of the interval, but I would be very sceptical if you told me that some property of black holes depends on excluded middle. Have you actually developed the analogous constructive theory, and found observational evidence to distinguish it from the classical one? This is, after all, what the experimental method says that you should do. Gauss tested Euclid's parallel postulate by surveying; two centuries later, such tests have been made astronomically, and the outcome was to overturn the once orthodox theory of geometry.

3.7. When we replace a component of a system, it needs to be backwards-compatible in its function, not necessarily its implementation or extent. After all, an architect who has been commissioned to replace a building will only do so with an exact copy if it is to be a museum.

In the case of general topology, the methods of construction in this paper are such that the new building must either be smaller than the old one (consisting of just locally compact spaces) or substantially bigger. This is entirely consistent with the historical development of the subject, which grew from figures embedded in Euclidean 3-dimensional space, to $\mathbb{R}^{\mathbf{n}}$, to projective and non-Euclidean geometry, to manifolds, to spaces of functions in analysis, and to domains for denotational semantics of programming languages. The intuition of continuity has been captured in numerous quite different ways, using metrics, uniformity and converge of sequences as well as systems of points and open neighbourhoods. Each axiomatisation leads to a different totality of domains of continuity.

For comparison, there can be little doubt that groups and fields have the right axioms for the intuitions that they seek to capture. This is indicated by the fact that we use the axioms directly for computation. Algebra textbooks are also able to classify finite fields, and they make a serious attack on the similar (albeit intractible) problem for groups.

The situation in general topology is much less clear. It is rather like a medieval "world" chart that more or less accurately depicts the Mediterranean, but has mythological creatures around the outside. The various approaches to continuity accurately capture real manifolds, just as the old cartographers recorded their own familiar territory, but we cannot be confident in using the outer parts of the chart.

The error in both cases is the co-ordinate system. Its assumptions provide reasonable approximations locally, but by their nature entail certain boundaries to the global system. Flat charts are useful, but Eratosthenes had known in ancient times, not only that the Earth is spherical (from lunar eclipses), but even how big it is. Similarly, it has been known since the 1960s that points and open sets are the wrong co-ordinate system for topology. Sheaves in algebraic geometry were based on open sets and not points, whilst algebraic topologists sought more "convenient" categories Bro64, Ste67, i.e. those that admit general spaces of functions. However, it is by no means clear what topologies these function-spaces should carry, especially if we want to invesigate the properties of $\mathbb{N}^{\mathbb{N}^{\mathbb{N}}}$ and $\mathbb{R}^{\mathbb{R}^{\mathbb{R}}}(c f . \S 12.10)$.

There is, therefore, nothing special about the boundaries of the category of objects that are called "topological spaces" in the textbooks. These books treat non-Hausdorff spaces with derision, and make little attempt to explore the full extent of even the world that is measured out by their own co-ordinate system. This was only begun when the analogy with the $\exists \wedge$-fragment of logic 
was recognised. We therefore undefine the terms "space" and "topological space", leaving them open to new definitions. The textbook spaces will be re-branded as Bourbaki spaces Bou66 to strip them of their authority.

3.8. Even when we have found the right co-ordinate system, it may not be appropriate to describe it in the same way for both the foundations and the applications. Any engineer knows that the user manual for a gadget should not be written in the same way as its technical specification. So this paper discusses the foundations of ASD, whilst [I, J] provide separate introductions that are suitable for its applications to elementary real analysis.

One reason for this is that the technical ideas may later be redeployed to make something else with an entirely different use. In our case, we shall find that there is a new underlying abstract structure that has other applications besides topology, and deserves to be studied in its own right ( $c f . \S \$ 612$ ). It includes certain definitions that, in the presence of the specifically topological structure, have different characterisations $(c f .87 .7)$. This is in line with the usual experience of applying category theory to mathematical disciplines, namely that ideas with various different traditional formulations turn out to be examples of a common abstract idea.

Secondly, the demands from users are often the driving forces behind advances in technology. However, as we have seen for computing hardware and software, the biggest improvements do not result from adding bells and whistles, but by re-thinking and strengthening the fundamental principles. In ASD, the principal challenge for the future is how to extend the boundaries beyond local compactness, in such a way that Banach spaces are given "the right" topology, whatever that means. In order to do this, it is the initial formulation of the underlying abstract structure in $\$ 6$, rather than its adaptation to topology, that needs to be replaced.

3.9. Finally, there is an issue for which architecture is entirely the wrong metaphor, and Columbus' departure from the Mediterranean is much more appropriate. Los Reyes Catolicos certainly did not promote free intellectual exploration in their domestic and colonial policy, but they did at least fund a "blue sea" project.

Nowadays, one is asked to give advance notice of all of the theorems that one intends to prove. Such planning may be possible when building a house, but it can be done if and only if there are no original ideas. A mathematician with a plan for a theorem wants to carry it out straight away, and the only pieces of equipment that are needed are a clear head and a clear blackboard. We don't put our lives at stake as Columbus did when we embark on scientific experiments or try to prove mathematical theorems, but if there is no intellectual risk of failure in a proposed piece of research, then it is redundant, and probably not worthy of funding.

We like to think that the finished product of mathematics is the most precise of any branch of science or engineering. The corollary of this is that the vision of a mathematical project in advance of its detailed plan is necessarily much more vague than in any other discipline.

And things may not go according to plan even if we do succeed, because there may be a new continent to discover. For a powerful account that has a far wider relevance than to physics, see Part IV of Smo06], which demonstrates a familiarity with the real experience of those who do revolutionary science that is absent from Kuh62.

\section{Stone duality}

Now we begin to describe the ideas from traditional general topology that we shall take as the "client's brief" for our new theory of that subject. We sketch the execution of this programme in the remainder of this paper. These ideas are presented in the form of universal properties, providing the leftmost arrow of the diagram in $\$ 3.1$. Following the horizontal arrow, we shall then 
develop a corresponding syntax. As far as possible, we do this in a general way that might be adapted to other subjects.

4.1. The principal mathematical insight that we shall employ is the duality between algebra and geometry.

Figures like circles and parabolae are defined by polynomials such as $x^{2}+y^{2}-a^{2}$ and $x^{2}-2 b y$, and superposition of the figures corresponds to multiplication of their polynomials. Like natural numbers, polynomials over an algebraically closed field may be expressed uniquely as products of irreducible or prime factors, which therefore capture the irreducible geometrical figures in a purely algebraic way.

By no means all commutative rings admit unique factorisation into primes, for example $3 \cdot 2=$ $6=(1+\sqrt{-5}) \cdot(1-\sqrt{-5})$ in $\mathbb{Z}[\sqrt{-5}]$. However, Ernst Kummer recovered the situation by introducing ideal numbers, defined as certain subsets of the ring. The notions of ideal and prime may be transferred from rings to lattices by following the analogy between their operations.

The systematic study of these ideas was undertaken by Marshall Stone [Sto37, Sto38. Since geometry and algebra each have their own natural generalities, they need not coincide exactly: it may be necessary to make extensions, restrictions or other adjustments on both sides to achieve agreement.

4.2. Putting Stone's programme in categorical language, let $\mathcal{A}$ be some category of "algebras" and $\mathcal{S}$ one of "spaces", the exact nature of which we leave open. Then by a Stone duality we mean an adjunction

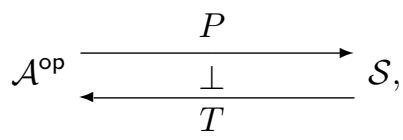

in which $T X$ is the algebra (maybe of open subspaces) associated with a space $X$, and $P A$ is the space of primes of an algebra $A$.

Because of the contravariance, the unit and counit of the adjunction $(\$ 2.4)$ go in the same direction in the categories $\mathcal{S}$ and $\mathcal{A}$,

$$
\eta_{X}: X \rightarrow P(T X) \quad \text { and } \quad \iota_{A}: A \rightarrow T(P A) .
$$

These say how each point defines a prime, and how the algebra is represented by its space of primes.

We call $X$ a sober space if $\eta_{X}$ is an isomorphism, i.e. every prime corresponds to a unique point, so a sober space is one that has exactly the points that are required by algebra. Similarly, $A$ is a spatial algebra if it has enough primes to provide a faithful representation, making $\iota_{A}$ invertible. The situation is a Stone equivalence if all spaces are sober and all algebras spatial, in which case the functors $P$ and $T$ are equivalences.

4.3. Stone dualities often arise from a schizophrenic object $\Sigma$, i.e. one that in some sense belongs to both categories, where

$$
T X \equiv \mathcal{S}(X, \Sigma) \quad \text { and } \quad P A \equiv \mathcal{A}(X, \Sigma) .
$$

The abstract description of Stone duality that we have given comes, essentially, from the book Joh82, which also provides an excellent and wide-ranging survey of concrete examples. In those that directly interest us, $\Sigma$ is at least a distributive lattice, whose elements we sometimes regard as "truth values" (\$77).

If you would like to apply the methods of this paper in another discipline, you will of course want to see what it can do for topology. However, on your second reading you should stop here. 
You need to identify the object $\Sigma$ in your subject. It is likely to be the most important algebraic or geometrical structure, albeit one that the undiscerning may dismiss as trivial (\$3.4).

Do not be misled by our discussion of subobject classifiers in \$7 or by the fact that all but two of the examples in [Joh82, §VI 4] are based on a two-element set - the other two use the line $\mathbb{R}$ and the circle $\mathbb{R} / \mathbb{Z}$. The natural numbers $\mathbb{N}$, integers $\mathbb{Z}$, complex numbers $\mathbb{C}$ and the closed interval $[0,1] \subset \mathbb{R}$ play this key role in other mathematical settings.

The most important property of $\Sigma$ that you should check is that all of the other desirable objects are sober with respect to it; this fact may already be a headline theorem in your subject. Then, ignoring the rest of $\$ 7$, it could be worth looking for an algebraic property like that in $\$ 7.7$. unlike sobriety, this may not have been recognised before.

4.4. The textbook definition of topological spaces gives rise to a Stone duality

$$
\mathcal{A} \equiv \mathbf{F r m} \equiv \operatorname{Loc}^{\text {op }} \quad \mathcal{A}^{\text {op }} \frac{P}{\frac{\perp}{T}} \mathcal{S} \quad \mathcal{S} \equiv \text { BbkSp }
$$

in which BbkSp is the category of Bourbaki spaces (what the textbooks call topological spaces, cf. 33.7 and continuous functions between them in the traditional sense.

The algebra $T X$ associated with a Bourbaki space is simply its lattice of open subspaces. Discarding the points, what kind of algebra is it? It has finite meets and arbitrary joins, where binary meets distribute over arbitrary joins. A frame is exactly an algebra for these operations, and Frm is the category of frames and homomorphisms.

The functor Frm $\rightarrow$ Set that forgets this algebraic structure has a left adjoint, which provides the free frame $\mathrm{F} N$ on a set $N$. The elements of the free frame may be characterised in two ways, either as upper families $\mathcal{U}$ of finite subsets of $N$, i.e. if $T \supset S \in \mathcal{U}$ then $T \in \mathcal{U}$ too [Joh82, II 1.2], or as Scott open families of arbitrary subsets of $N$.

How do we recover the points or primes of a frame $A$ ? They are continuous maps $1 \rightarrow P A$, which correspond under the adjunction to frame homomorphisms $A \rightarrow T \mathbf{1}$. Since $T \mathbf{1} \cong \mathcal{P}(\mathbf{1})$, these are subsets $F \subset A$ whose membership predicate is a frame homomorphism, so $\top \in F$; if $\alpha, \beta \in F$ then $\alpha \wedge \beta \in F$; and if $\bigvee \alpha_{i} \in F$ then $\alpha_{i} \in F$ for some $i$.

4.5. In our investigation of topology, we shall often find it helpful to consider the analogy with discrete objects ("sets" in the sense of $\$ 2.10$ ). Classically, there is a Stone equivalence $\mathbf{C A B A}{ }^{\mathrm{op}} \simeq$ Set, where CABA is the category of complete atomic Boolean algebras and homomorphisms for $\bigvee$ and $\Lambda$, for which $T X$ is the powerset of the set $X$ and $P A$ is the set of atoms (minimal non- $\perp$ elements) of $A$. This equivalence was proved by Adolf Lindenbaum and Alfred Tarski Tar35].

4.6. We want to design a new building to replace the old one functionally and not extensionally (\$3.7), so it is really the underlying intuition of Stone duality that we shall use. The concrete examples can be a little distracting, especially with regard to the notion of sobriety. We defined this above in a way that depends on the choice of adjunction, and so may vary from one application to another.

Classically, $X$ is a sober space with respect to the concrete Stone duality between frames and Bourbaki spaces (4.4) iff every irreducible closed subspace is the closure of a unique point. In particular, every Hausdorff space is sober in this sense [Joh82, II 1.6]. On the other hand, we shall see that the Hausdorff spaces $\mathbb{N}$ and $\mathbb{R}$ are sober with respect to our abstract Stone duality iff they admit definition by description (\$9.11) and by Dedekind cuts (\$11.11) respectively.

There is no theorem to say that the old and new definitions of sobriety are coextensive, simply because there is no common generality of which these are species. There is nothing to be gained by trying to find such a generality, as it would be artificial. 
4.7. One way to achieve Stone equivalence is just to replace $\mathcal{S}$ by $\mathcal{A}^{\text {op }}$. In the cases where algebras are frames or commutative rings, the new "algebraic" spaces are called locales or affine varieties, respectively. A continuous function $f$ between the locales that correspond to frames $A$ and $B$ is simply a frame homomorphism $B \rightarrow A$ in the opposite direction. However, since any frame homomorphism preserves (finite meets and) all joins, it has a right adjoint, and these are written $f^{*} \dashv f_{*}$.

Stone duality is now vacuous as an extensional theorem, instead serving as a vehicle for the intuitions of general topology and algebraic geometry, which may be used to reconstruct these disciplines in a new, algebraic, form [Joh82, Har77.

Locale theory removes many of the evils of point-set topology. For example, it is largely free of the axiom of choice, without which it is next to impossible to do anything with Bourbaki spaces. In particular, Peter Johnstone proved Tychonov's theorem (that a product of compact spaces is compact) without it Joh82, Thm. III 1.7]. Also, the closed interval [0,1] is Dedekind complete and compact $(\$ 1.7)$ in the localic reals over any elementary topos, whereas if we interpret the point-based definitions in the internal language of a sheaf topos, the object of Cauchy reals is typically smaller than the Dedekind one, and the Heine-Borel theorem fails [FH79.

4.8. Whilst locale theory makes significant advances over traditional topology it still relies on the old foundations of the category of sets, as of course do the other concrete examples in this section. But our goal is to build new foundations for topology.

Nevertheless, locales provide a useful test-bed for many of the ideas that we shall consider. We shall in particular need to know about sublocales $i: S \longmapsto X$, by which we mean coequalisers of frames. These have $i_{*} \cdot i^{*}=\mathrm{id}$, and are therefore captured by the other composite, $j \equiv i^{*} \cdot i_{*}$, which satisfies id $\leqslant j=j \cdot j$ and preserves $\wedge$, but usually not any kind of joins. An endofunction $j$ of a frame with these properties is called a nucleus, and always arises from a sublocale in this way Joh82, II 2.2]. In particular, an element $a \in A$ of a frame gives rise to the nuclei $a \Rightarrow(-)$ and $a \vee(-)$, which encode the corresponding open and closed sublocales, respectively. We shall encounter localic nuclei in $\$ \$ 5.10,6.6$ and 7.5

\section{Always topologize}

5.1. We do not require underlying sets for the category $\mathcal{S}$, because the new notion of "space" is intended to be an unknown. However, algebras need carriers. The fundamental idea of Abstract Stone Duality is to use the (as yet, unknown) spaces as carriers for the algebras. In particular, in topology, we regard the lattice of open subspaces of a space as another space, following another methodological principle due to Marshall Stone: "always topologize" Sto38.

For Stone equivalence, $\mathcal{A} \simeq \mathcal{S}^{\text {op }}$. So we are saying that we want $\mathcal{S}^{\text {op }}$ to be a category of algebras over $\mathcal{S}$. For this, we need a way of formulating (potentially infinitary) algebraic theories that works over an arbitrary category $\mathcal{S}$, and not just over the category of sets. Such an account is provided by the categorical notion of monad [Lin69].

In 8 we shall give some of the formal definitions for monads, as adapted to ASD, and the full treatment is of course given in the papers cited. However, our purpose here is to tell the story behind these ideas. 
5.2. Recall that concrete Stone dualities often arise from a "schizophrenic" object $\Sigma$, for which $T X \equiv \mathcal{S}(X, \Sigma)$, whilst, by the new hypothesis, $T X$ is also to be an object of $\mathcal{S}$. So it is natural to ask that $\mathcal{S}$ have all exponentials of the form $\Sigma^{X}$, by which we mean that $\mathcal{S}$-maps $\Gamma \times X \rightarrow \Sigma$ are to be in natural bijection with those $\Gamma \rightarrow \Sigma^{X}$ (2.5). A pre-requisite for this definition is that $\mathcal{S}$ have finite products, 1 and $\times$. Note that we are asking just for powers of $\Sigma$ (and, consequently, of its powers too), but not of general objects. In other words, at least at this stage, we are not saying that the category should be cartesian closed (but see 12.9 .

Nevertheless, any object $\Sigma$ that has powers gives rise to an adjunction

$$
\left.\Sigma^{(-)}\right|_{\mathcal{S}} ^{\mathcal{S}^{\text {op }}} \overbrace{}^{\dagger} \Sigma^{(-)}
$$

which we write vertically in order to avoid confusing it with the one in $\$ 4.2$ for Stone duality. This adjunction induces a monad on $\mathcal{S}$, so let $\mathcal{A}$ be its category of Eilenberg-Moore algebras, for which the standard theory gives a comparison functor $\mathcal{S}^{\text {op }} \rightarrow \mathcal{A}$ [EM65] Tay99, 7.5.3(c)]. Formulating abstract Stone duality as an axiom on $\mathcal{S}$, we ask that this be an equivalence.

As we want to develop a formalism inspired by the $\lambda$ - and predicate calculi ( $\$ 2$ ), and to get away from that of set theory, we shall use Greek letters $(\phi, \psi, \theta)$ for terms of type $\Sigma^{X}$, which will represent open subspaces. Then, instead of writing $x \in U$ for membership, we use $\lambda$ application, $\phi x$. (We shall re-employ the set-theoretic notation for more general kinds of subspaces than open ones in $\S \S 6.7 \& 12.8$.)

5.3. Before making things any more abstract, let's link this idea back to the concrete case of set theory instead of topology ( $c f . \$ 2.10$ ). The Lindenbaum-Tarski theorem ( 4.5 ) is strictly classical, but Robert Paré proved a categorical version of it that is valid any elementary topos $\mathcal{S}$, i.e. (essentially) in any model of intuitionistic Zermelo set theory. The powerset $\mathcal{P}(X)$ is given by the exponential $\Omega^{X}$, where $\Omega$ is the lattice of truth values, which we shall discuss further in $\$ 7$. Paré showed that the contravariant functor $\Omega^{(-)}$is monadic in the above sense Par74.

We find limits of algebras by computing them for their carriers and lifting the algebraic structure; in categorical jargon, the functor $U: \mathcal{A} \rightarrow \mathcal{S}$ creates limits. Since $\mathcal{A} \simeq \mathcal{S}^{\mathrm{op}}$, this derives colimits in $\mathcal{S}$ from its limits. Paré's theorem therefore simplified the definition of an elementary topos, which then only needed to assert the existence of limits and not colimits.

5.4. This result was also the original inspiration for ASD, in 1993, although the results that we discuss in the rest of this section were discovered later.

How could we set up the monadic situation in topology, within the category of Bourbaki spaces? Ralph Fox's original investigations [Fox45] showed that the function-space $Y^{X}$ only behaves reasonably well when $X$ is locally compact. The meaning of "reasonably well" crystalised into the notion of a cartesian closed category that emerged in the 1960s and was shown to be equivalent to the $\lambda$-calculus $(\S \$ 2.2 \& 2.5$. Meanwhile, it was becoming clear that, for a compact subspace, the filter of open neighbourhoods (\$5.7) plays a more important role than the set of points Wil70].

Then Dana Scott saw that the crucial case is the function-space $\Sigma^{X}$, where $\Sigma$ is the Sierpiński space $(\$ 7) . \Sigma^{X}$ is the lattice of open subsets of $X$, but it obeys the universal property in $\$ 2.5$ iff $X$ is locally compact and $\Sigma^{X}$ has the non-Hausdorff topology that now bears Scott's name [Sco72]. The study of continuous lattices $\left[\mathrm{GHK}^{+} 80\right.$ and domain theory grew out of this. For a historical study of function spaces in topology, see Isb86.

The category of locally compact spaces has products and an object $\Sigma$ that has powers $\Sigma^{X}$, as we require. Whilst general functions-spaces $Y^{X}$ exist as Bourbaki spaces when $X$ is locally compact, the result need no longer be locally compact, so we do not have a cartesian closed 
category. In particular, $\mathbb{N}^{\mathbb{N}}$, known as Baire space, is not locally compact, essentially because its open subspaces are rather large, whilst its compact ones are rather small.

Nevertheless, cutting down to sober spaces, we have a model of the monadic situation above, as we show in $\S \$ 5.8 \mathrm{ff}$.

5.5. Category theory is a very potent drug, which should only be prescribed in small quantities. A theorem of Jon Beck [Tay99, §7.5] (he never published it himself) characterises when an adjunction $\mathrm{F} \dashv \mathrm{U}$ is monadic in terms of two properties of the right adjoint $\mathrm{U}: \mathcal{A} \rightarrow \mathcal{S}$ :

First, $\mathrm{U}$ must reflect invertibility: for $f: A \rightarrow B$ in $\mathcal{A}$, if $\mathrm{U} f$ has an inverse in $\mathcal{S}$ then $f$ already had one in $\mathcal{A}$. We shall see in $\$ 6.2$ that this is equivalent to our abstract definition of sobriety.

Second, U must create U-split coequalisers (cf. $\$ 5.3$ ). New students balk at this clause, but it is the active ingredient in this dose of category theory. We need to explain $\Sigma$-split equalisers, as the coequalisers become under the contravariance. These are subspaces $i: S \subset X$ that have the subspace topology, i.e. any open subspace of $S$ is the restriction of one of $X$, but in a canonical way. That is, there is a map $I: \Sigma^{S} \rightarrow \Sigma^{X}$ for which $I \cdot \Sigma^{i}=\mathrm{id}_{\Sigma^{S}}$.

Beck's theorem says that, given an idempotent $E$ on $\Sigma^{X}$ that ought to define a $\Sigma$-split subspace of $X$, then it does, i.e. there are $i$ and $I$ for which $E=I \cdot \Sigma^{i}$. We shall give the formal definitions in $\$ 6.5$

5.6. The simplest (and first, [C]) examples of $\Sigma$-split subspaces are open and closed ones.

An open subsubspace $V \subset U \subset X$ of an open subspace is already an open subspace of $X$. Conversely, the inverse image of $W \subset X$ in $U \subset X$ is the intersection, $U \cap W$. Then the operation $U \cap(-)$ provides $\Sigma^{i}: \Sigma^{X} \rightarrow \Sigma^{U}$ for the inclusion $i: U \subset X$, and its splitting $I: \Sigma^{U} \rightarrow \Sigma^{X}$ is the inclusion $(V \subset U) \mapsto(V \subset X)$. The composite $E \equiv I \cdot \Sigma^{i}$ is the idempotent $U \cap(-)$ on $\Sigma^{X}$. In the ASD notation, $U$ and $V$ are represented by their classifiers $\theta$ and $\phi$, and then $E$ is $\lambda \phi . \phi \wedge \theta$.

In the case of closed subspaces, any open subsubspace $V \subset C \subset X$ is also the intersection with $C$ of some open subspace of $X$. But in this case the most convenient choice of representative is the maximal one, $V \cup U$, where $U$ is the open complement of $C$. This provides $I$, whilst $\Sigma^{i}$ is represented by $U \cup(-)$. Now $E \equiv I \cdot \Sigma^{i}$ is $U \cup(-)$ or $\lambda \phi . \phi \vee \theta$ on $\Sigma^{X}$.

5.7. Before giving further examples of $\Sigma$-split subspaces, we need to say something about compact ones. Recall that a subspace $K \subset X$ of a Bourbaki space is compact if, whenever $K \subset \bigcup_{i} U_{i}$ for some family of open subspaces, this already holds for some finite subfamily. This says exactly that the predicate $K \subset U$ is Scott continuous as $U$ ranges over open subspaces of $X$. The result lies in the object of truth values, considered as a topological space, so the predicate is a continuous function $A: \Sigma^{X} \rightarrow \Sigma$.

The operator $A$ obeys the Gentzen-style rule ( $c f$. 2.1 ) for universal quantification of any predicate $\phi$ over $K$, so long as $\phi$ denotes an open subspace:

$$
\frac{\Gamma, x: X, x \in K \vdash \phi x \Leftrightarrow \top}{\Gamma \vdash A \phi \Leftrightarrow \top}
$$

In particular, if $K=X$ then $A$ is the universal quantifier $\forall$, whilst if it is a subspace we sometimes write $A$ as a modal operator $\square$, which we read as "must" or "necessarily". We explain in $\$ 12$ why we use a symbol $\forall$ instead of $\forall$.

5.8. The definitions of local compactness for Bourbaki spaces and locales naturally express the object in question as a $\Sigma$-split subspace of $\Sigma^{N}$, where $N$ is any set, which we may think of as $\mathbb{N}$ or a cardinal. Then $\Sigma^{N}$ is the powerset of $N$, or the topology on $N$ considered as a discrete space, where $\Sigma^{N}$ is itself equipped with the Scott topology.

According to the definition for not necessarily Hausdorff spaces in HM81, if a point lies in an open subspace $(x \in U)$ then there is a compact subspace $K$ and an open one $V$ such that $x \in V \subset K \subset U$. Moreover, there is a family of such pairs $(V, K)$, indexed by a set $N$. 
Now let $A_{n}: \Sigma^{X} \rightarrow \Sigma$ or $A_{n}: \Sigma^{\Sigma^{X}}$ be the Scott-continuous operator corresponding to the subspace $K^{n}$. Meanwhile, the open subspaces $U$ and $V^{n}$ are represented in our notation by $\phi, \beta^{n}: \Sigma^{X}$. (We use super- and subscripts to indicate whether these subspaces or terms increase or decrease as the index $n: N$ increases.)

Hence local compactness says that

$$
U=\bigcup\left\{V^{n} \mid K^{n} \subset U\right\} \quad \text { or } \quad \phi=\exists n . \beta^{n} \wedge A_{n} \phi,
$$

which we call the basis decomposition. Then the maps $i: X \rightarrow \Sigma^{N}$ and $I: \Sigma^{X} \rightarrow \Sigma^{\Sigma^{N}}$, defined by

$$
i x \equiv \lambda n \cdot \beta^{n} x \quad \text { and } \quad I \phi \equiv \lambda \xi . \exists n . \xi n \wedge A_{n} \phi
$$

satisfy $\Sigma^{i} \cdot I=\mathrm{id}_{\Sigma^{X}}$.

5.9. A locally compact locale is one whose corresponding frame is a continuous lattice Joh82, $\S$ VII 4]. This relativises the notion of compactness to a Scott-continuous relation $\beta \ll(-)$ (called way below) on open subsets, and then requires that

$$
\phi=\bigvee\left\{\beta^{n} \mid \beta^{n} \ll \phi\right\}, \quad \text { or } \quad \phi=\exists n . \beta^{n} \wedge A_{n} \phi, \quad \text { where } \quad A_{n} \phi \equiv\left(\beta^{n} \ll \phi\right),
$$

which is another basis decomposition, giving $i$ and $I$ as before.

These two definitions are actually different, in that

(a) $A_{n} \top=\top$ and $A_{n}(\phi \wedge \psi)=A_{n} \phi \wedge A_{n} \psi$ in the case of Bourbaki spaces, whilst

(b) the indices $n \in N$ form a lattice in the localic situation.

However, a space only carries a basis with both properties if it is itself compact and the intersection of any two compact subspaces is again compact; it is then called stably locally compact. See G, JKM01 for the details of this approach to local compactness.

5.10. We have shown that every locally compact Bourbaki space or locale is a $\Sigma$-split subspace of $\Sigma^{N}$. So if we can prove that all such subspaces are locally compact locales then we have a model of the monadic situation. Locally compact locales and sober locally compact Bourbaki spaces are equivalent, assuming the axiom of choice.

Recall from $\$ 4.4$ that the Scott topology on $\Sigma^{N}$ is the free frame $\mathrm{F} N$ on the set $N$. For a $\Sigma$-split subspace $X \subset \Sigma^{N}$, the topology $L$ on $X$ is a retract of $\mathrm{F} N$, where $H: \mathrm{F} N \rightarrow L$ and $I: L \longmapsto \mathrm{F} N$ are Scott-continuous and satisfy $H \cdot I=$ id. Hence $L$ is a continuous lattice Joh82, VII 2.3].

The maps $I$ and $H$ need not be adjoint, but since $H$ is a frame homomorphism, it has a right adjoint $R$ that is monotone but not necessarily Scott continuous. Then id $\leqslant j \equiv R \cdot H=j \cdot j$ preserves meets, making it a nucleus $\left(4.8\right.$, and $H \equiv i^{*}$ and $R \equiv i_{*}$, where $i: X \subset \Sigma^{N}$ is the sublocale defined by $j$.

5.11. Although we have now related Beck's theorem in category theory to ideas of general topology, these are still rather abstract. We need a more compelling example from the applications of topology to demonstrate that $\Sigma$-split subspaces are important to the customers of this subject (cf. 33.5 . Recall from $\S \$ 1.7$ and 4.7 that the classical Heine-Borel theorem fails in Russian Recursive Analysis but holds in locale theory.

Now, it is possible to construct a "space of Dedekind cuts" in any category that has $\mathbb{N}$, powers of $\Sigma$, equalisers and some other properties. However, the "closed interval" $[0,1]$ within this space need not be compact. The category of dcpos and Scott-continuous functions has the relevant structure, but the order on a dcpo determines its topology, and the "real line" is (uncountable but) discrete in both senses, so its only compact subspaces are finite sets. 
5.12. Beck's theorem comes to the rescue.

The classical Heine-Borel theorem provides a Scott-continuous $\Sigma$-splitting $I$ for the equaliser $i: \mathbb{R} \longmapsto \Sigma^{\mathbb{Q}} \times \Sigma^{\mathbb{Q}}$, where $i x \equiv(\lambda d . d<x, \lambda u . x<u)$. In traditional notation, the map $I$ is given by

Or

$$
\begin{gathered}
(V \subset \mathbb{R}) \text { open } \mapsto\{(D, U) \mid \exists d \in D . \exists u \in U .(d<u) \wedge[d, u] \subset V\}, \\
\phi: \Sigma^{\mathbb{R}} \mapsto \lambda \delta v . \exists d u . \delta d \wedge v u \wedge(d<u) \wedge \forall x:[d, u] . \phi x
\end{gathered}
$$

in the ASD $\lambda$-calculus.

The idempotent $I \cdot \Sigma^{i}$ can be shown to be equal to an expression that involves the rationals alone, so we have an example of the hypothesis of Beck's theorem, and hence an object $R$ of ASD. In $R$, the interval $[0,1]$ is compact, and indeed this object satisfies all of the properties that one could reasonably require of a computable real number object [I. Looking more closely at its topology, $\Sigma^{R}$, we find, as in the classical situation, that any open subspace is a countable disjoint union of open intervals, although the words "countable" and "interval" require some constructive qualification [J].

We now have enough experimental evidence to start writing down some axioms $(\$ 3.2)$, at least for locally compact spaces.

\section{The monadic framework}

In the previous section we described a number of properties in topology that are related to the monadic adjunction $\Sigma^{(-)} \dashv \Sigma^{(-)}$. We also showed that this is satisfied when $\Sigma$ is the Sierpiński space in the category of locally compact spaces, or the subobject classifier in any topos, and in these cases $\Sigma^{X}$ is the topology or powerset of $X$. In this section we describe the purely categorical structure that we abtract from this situation. Then we shall show how it can can be formulated as a new symbolic language. See $[\mathrm{A}, \mathrm{B}$, for the details in ASD.

6.1. The category $\mathcal{S}$ must have

(a) finite products, $\mathbf{1}$ and $\times$;

(b) an object $\Sigma$ with a map $\star: 1 \rightarrow \Sigma$ ( $\star$ is only used in 6.10 ; and

(c) powers $\Sigma^{X}$ for all $X \in$ obS ; then

(d) the adjunction $\Sigma^{(-)} \dashv \Sigma^{(-)}$must be monadic.

In investigating this structure, we shall need to apply the (contravariant) functor $\Sigma^{(-)}$repeatedly, giving some unwieldy towers of exponentials. For this reason, we often write

$$
\Sigma^{2} X \equiv \Sigma^{\Sigma^{X}} \quad \Sigma^{3} X \equiv \Sigma^{\Sigma^{\Sigma^{X}}} \quad \Sigma^{4} X \equiv \Sigma^{\Sigma^{\Sigma^{\Sigma^{X}}}} \ldots
$$

The unit of the adjunction, $\eta_{X}: X \rightarrow \Sigma^{2} X$, is the transpose of the co-unit, $\varepsilon_{X} \equiv \mathrm{ev}_{X}: \Sigma^{X} \times X \rightarrow$ $\Sigma$, which is in turn the other transpose of id : $\Sigma^{X} \rightarrow \Sigma^{X}$. Then $\eta$ is part of the structure of the monad, for which $\Sigma^{2} X$ is the free algebra on an object $X$, with structure map $\mu_{X} \equiv \Sigma^{\eta_{X}}$, where $\mu$ is called the multiplication.

The corresponding symbolic language is the familiar one with pairing and $\lambda$-abstraction, except that the exponential type $S^{X}$ and corresponding $\lambda$-terms $\lambda x$.s may only be formed in the case where $S$, the type of the body $s$, is a power of $\Sigma$. Then

$$
\eta_{X} x \equiv \lambda \phi . \phi x \quad \text { and } \quad \mu_{X} \mathcal{F} \equiv \lambda x . \mathcal{F}(\lambda \phi . \phi x)
$$

define the monad. Contexts (\$2.6) in the first version of this language just consist of typed variables, but we shall need to add equational hypotheses in $\S 8.5 \& 9.10$. 
6.2. The first clause of Beck's theorem (\$5.5(a)) says that if $\Sigma^{f}: \Sigma^{Y} \rightarrow \Sigma^{X}$ is invertible then so is $f: X \rightarrow Y$ itself. This is equivalent to requiring, for every object $X$, that $\eta_{X}$ be the equaliser of the parallel pair in the diagram,

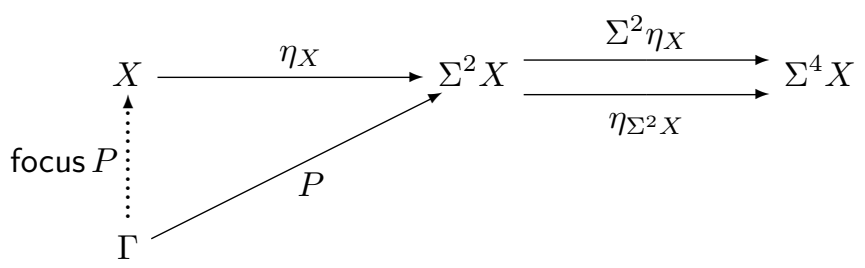

In other words, if $P$ also has equal composites then there is a unique map that makes the triangle commute.

$P$ has equal composites iff its transpose, $\tilde{P}: \Sigma^{X} \rightarrow \Sigma^{\Gamma}$, is a homomorphism of Eilenberg-Moore algebras for the monad. Sobriety then says that $\tilde{P}=\Sigma^{\text {focus } P}[\mathrm{~A}, \S 4]$.

6.3. According to the technique of $\$ 2.8$ a morphism $P: \Gamma \rightarrow \Sigma^{2} X$ is the same thing as a term $\Gamma \vdash P: \Sigma^{2} X$. This has equal composites with the parallel pair above iff

$$
\Gamma, \mathcal{F}: \Sigma^{3} X \vdash \mathcal{F} P=P(\lambda x . \mathcal{F}(\lambda \phi . \phi x)): \Sigma .
$$

We call $P$ prime if this holds, extending the usage of this word beyond that in 4.1

Here we have our first example of the difference between the definitions that we give for the foundations and the applications ( $\$ 3.8$ ): when we add the other topological structure to the theory, primality can be characterised more simply by saying that $P$ preserves $T, \perp, \wedge$ and $\vee(11.6)$.

The object $X$ is sober iff every prime $P$ has a unique fill-in

$$
\Gamma \vdash \text { focus } P: X \quad \text { such that } \quad \Gamma, \phi: \Sigma^{X} \vdash \phi(\text { focus } P)=P \phi: \Sigma \text {. }
$$

Following 2.11 , we turn this universal property into a new system of symbolic rules. Indeed, we have just given the introduction and $\beta$-rules for focus [A, $\S 8$.

The operation in the elimination rule takes $a: X$ to $\lambda \phi . \phi a: \Sigma^{2} X$. This is prime: categorically, $\eta_{X}$ has equal composites with the parallel pair, by naturality of $\eta_{(-)}$with respect to $\eta_{X}$, but one could also check this by a $\lambda$-calculation. Finally, the $\eta$-rule is focus $(\lambda \phi . \phi a)=a: X$.

The normalisation theorem ( $c f . \$ 2$ says that focus may be eliminated from any term $\phi$ : $\Sigma^{X}$, whilst any term of ground type is provably equivalent to focus $P$, where $P$ does not itself involve focus.

The symbolic calculus extended with focus corresponds (via $\$ 2.8$ again) to a certain category. By the normalisation theorem, this has the same objects (contexts) as the original one, but its morphisms $\Gamma \rightarrow \Delta$ are in bijection with the Eilenberg-Moore homomorphisms $\Sigma^{\Delta} \rightarrow \Sigma^{\Gamma}$, A, $\S \S 6-7]$.

6.4. It is pertinent to ask of this $\beta$-rule how much of the expression surrounding focus $P$ is to be taken as $\phi$, and moved inside $P \phi$. That is, for any $F: \Sigma^{\Sigma}$, does

$$
F(\phi(\text { focus } P)) \quad \text { become } \quad F(P \phi) \quad \text { or } \quad P(\lambda x . F(\phi x)) \text { ? }
$$

So long as $P$ is prime, this doesn't matter, because the two results are equal (consider $\mathcal{F} \equiv$ $\lambda Q . F(Q \phi)$ in the definition of primality).

Hayo Thielecke Thi97] considered an operation called force with the same $\beta$-rule, but without the primality side condition. Now it does matter where we draw the boundary of the super-term $\phi$ : the computational effect is to pass $\phi$ as an argument to $P$, and then jump to the continuation $F$ when (if) $P$ returns. In order to study computational effects in general, Thielecke developed categorical machinery that is very similar to our account of abstract sobriety, but independently. 
6.5. The second clause of Beck's characterisation ( $\$ 5.5$ brings in all algebras that are definable à la Eilenberg-Moore. It says that certain equalisers exist, and are taken by $\Sigma^{(-)}$to coequalisers B. $\S \S 2-4]$.

Our approach to this is to spell out what was meant in $\$ 5.5$ by "data on $Y$ that ought to define a $\Sigma$-split subspace". For this, it is enough to consider equalisers of the form

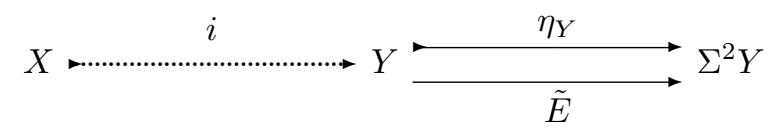

that become split by $\eta_{\Sigma^{Y}}$ when we apply $\Sigma^{(-)}$. This meas that, in the diagram

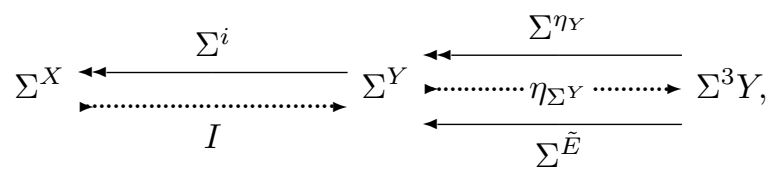

the equation $\Sigma^{\tilde{E}} \cdot \eta_{\Sigma^{Y}} \cdot \Sigma^{\tilde{E}}=\Sigma^{\tilde{E}} \cdot \eta_{\Sigma^{Y}} \cdot \Sigma^{\eta_{Y}}$ holds. Besides asking for the equaliser $i: X \longmapsto Y$ in (a), we further require that the solid lines in diagram (b) form a coequaliser. For this we simply need a map $I$ such that

$$
I \cdot \Sigma^{i}=E \equiv \Sigma^{\tilde{E}} \cdot \eta_{\Sigma^{Y}}
$$

Whilst these conditions may look very strange, they are actually adapted from the definition of an Eilenberg-Moore algebra $(A, \alpha)$ : with $Y \equiv \Sigma^{A}, \tilde{E} \equiv \Sigma^{\alpha}$ and $I \equiv \eta_{A}$, they yield $P A \equiv X$. Conversely, one can show that the category of algebras admits coequalisers of this form. However, the category of spaces that we have axiomatised here need not have all equalisers, for example LKLoc does not.

6.6. Writing the equation for $\tilde{E}$ in the $\lambda$-calculus $(2.8)$, we call $E: \Sigma^{Y} \rightarrow \Sigma^{Y}$ a nucleus if

$$
\mathcal{F}: \Sigma^{3} Y, y: Y \vdash E\left(\lambda y^{\prime} . \mathcal{F}\left(\lambda \psi \cdot E \psi y^{\prime}\right)\right) y=E\left(\lambda y^{\prime} \cdot \mathcal{F}\left(\lambda \psi \cdot \psi y^{\prime}\right)\right) y,
$$

observing the extra $E$ on the left hand side. Since we don't want to deal with dependent types, we do not allow $E$ to have parameters.

We have appropriated this name from locale theory $(\$ 4.8)$, since both kinds of nucleus are used to define subspaces. The definitions are not the same, but the use of different letters $(E$ and $j)$ should avoid ambiguity. Like primes $(\$ 6.3)$, nuclei in ASD have another characterisation in the presence of the full topological structure (\$11.6), namely

$$
E(\phi \wedge \psi)=E(E \phi \wedge E \psi) \quad \text { and } \quad E(\phi \vee \psi)=E(E \phi \vee E \psi)
$$

In this setting, ASD nuclei are Scott continuous, whilst those in locale theory need only be monotone; on the other hand, localic nuclei satisfy id $\leqslant j$, which is not required in ASD. Beware in particular that the ASD nucleus for an open subspace is given by $E \phi \equiv(\theta \wedge \phi)(c f$. $\$ 5.6)$, whilst the localic one is $j \phi \equiv(\theta \Rightarrow \phi)$ (\$4.8). Nevertheless, there are some important examples that satisfy all of the conditions of both definitions [I, §8] [L]. 
6.7. The symbolic calculus ( $\$ 2.11$ that corresponds to Beck's theorem has to define both the equaliser of the main types and also the coequaliser of their topologies. It does this using two sets of rules [B, §8].

A term $\Gamma \vdash a: Y$ has equal composites with the parallel pair in 6.5 (a) iff

$$
\Gamma, \psi: \Sigma^{Y} \vdash \psi a=E \psi a: \Sigma,
$$

and then we say that it is admissible. The introduction rule for the equaliser, i.e. its universal property, then allows us to form

$$
\Gamma \vdash \text { admit } a:\{Y \mid E\},
$$

whilst the inclusion $i$ is the elimination rule. The $\beta$ - and $\eta$-rules are

$$
\Gamma \vdash i(\operatorname{admit} a)=a: Y \quad \text { and } \quad x:\{Y \mid E\} \vdash \operatorname{admit}(i x)=x:\{Y \mid E\} \text {. }
$$

We also have to ensure that the second diagram $(6.5(b))$ is a coequaliser, using another set of rules. We get the introduction rule for free, as the map $\Sigma^{i}: \Sigma^{Y} \rightarrow \Sigma^{\{Y \mid E\}}$. The elimination rule says that $\Sigma^{i}$ is split by another map $I$, so $I \cdot \Sigma^{i}=E$ and $\Sigma^{i} \cdot I=\mathrm{id}_{\{Y \mid E\}}$. These equations are the $\beta$ - and $\eta$-rules

and

$$
\begin{gathered}
y: Y, \psi: \Sigma^{Y} \vdash I(\lambda x . \psi(i x)) y=E \psi y \\
x:\{Y \mid E\}, \phi: \Sigma^{\{Y \mid E\}} \vdash I \phi(i x)=\phi x .
\end{gathered}
$$

The normalisation theorem for this calculus $(c f . \$ 2.12$ ) says that every type can be embedded as a subspace of a type formed without comprehension, and terms also normalise in a simple way. This makes the corresponding category equivalent to the opposite of the Eilenberg-Moore category $[B$, $\S \S 9-10]$.

6.8. Any map $f: X \rightarrow Y$ that is $\Sigma$-split, i.e. with $F: \Sigma^{X} \rightarrow \Sigma^{Y}$ such that $F \cdot \Sigma^{f}=\mathrm{id}_{\Sigma^{X}}$ or $F \phi(f x)=\phi x$, agrees up to isomorphism with the subspace $\{Y \mid E\}$ with $E \equiv \Sigma^{f} \cdot F$. In particular, $f$ is regular mono. The isomorphism is

$$
\begin{array}{lll}
x: X & \vdash & x^{\prime} \equiv \operatorname{admit}(f x):\{Y \mid E\} \\
x^{\prime}:\{Y \mid E\} & \vdash & x \equiv \text { focus }_{X}\left(\lambda \phi: \Sigma^{X} \cdot F \phi\left(i x^{\prime}\right)\right): X .
\end{array}
$$

To show that these maps are mutually inverse, you need the rules above, together with

$$
i\left(\text { focus }_{X} P\right)=\text { focus }_{Y}\left(\Sigma^{2} i P\right),
$$

first proving that if $P$ is prime then so is $\Sigma^{2} f P$. This uses sobriety of $X$ and $Y$.

6.9. Notice that the $\Sigma^{\{\}} \beta$-rule is the only one that introduces $E$ into terms. This is something that we want to avoid if at all possible, because the expressions for nuclei are usually very complicated. When $E$ does find its way into a program, it will give rise to a substantial computation.

Actually, this is what we expect from practical considerations. Consider, for example, the nucleus that defines $\mathbb{R}$ in ASD (\$5.12). The universal quantifier that says that the interval is compact is given by

$$
\forall x:[0,1] . \phi x \equiv I \phi(\lambda d . d<0, \lambda u . u>0),
$$

which applies the extension $I \phi$ of $\phi$ to the pseudo-Dedekind cut that represents the interval. This can be computed by dividing the interval up into sufficiently small parts, on each of which the predicate $\phi$ must be satisfied in the fashion of Ramon Moore's interval analysis [Moo66], i.e. by evaluating the arithmetical operations on the (endpoints of) the subinterval, instead of using single real values [Bau08, [I, $\mathrm{K}$. 
6.10. How common is the monadic situation? We may obtain it from any category $\mathcal{S}_{0}$ with an object $\Sigma_{0}$ that has powers $\Sigma_{0}^{X}$. Let $\mathcal{A}$ be the Eilenberg-Moore category for the monad on $\mathcal{S}_{0}$; then $\mathcal{S} \equiv \mathcal{A}^{\text {op }}$ has the monadic property. The proof of this is very easy — apart from the most basic requirement, namely that $\mathcal{S}$ have products (coproducts of algebras) [B, $\S 7]$.

Can other categorical structure be defined in $\mathcal{S}$, before we add specifically topological features? We have already remarked in $\$ 5.3$ that colimits come for free, as long as we have the corresponding limits, so $\mathcal{S}$ has coproducts and $\Sigma$-split coequalisers. In fact, the coproducts are stable and disjoint, i.e. the category $\mathcal{S}$ is extensive $(c f . \$ 9.1)$. Note that $\mathbf{0} \longmapsto \mathbf{1}$ is $\Sigma$-split iff $\Sigma$ has a point $(\$ 6.1(\mathrm{~b}))$. Whilst extensivity is a purely categorical statement, its proof relies heavily on the new $\lambda$-calculus [B, $\S 11]$.

6.11. Thielecke argues [Thi97 that $\Sigma$ (the "answer type" $R$, in his notation) needs no extra structure. It may perhaps be seen as a free type variable, or one that is quantified at the outermost level. I once said to him that his work must therefore be either very superficial or very deep. The fact that one can develop quite a lot of theory from just this monadic adjunction $\mathcal{S}^{\text {op }} \leftrightarrows \mathcal{S}$, or from pure equideductive logic ( $c f . \$ 12.5$, without any other hypothesis on $\Sigma$, leads me to believe more and more in the second possibility.

This structure will provide the skeleton on which the more obviously topological structure is the flesh. Dressed in a different way, I also believe that it could be applicable to game semantics of sequential computation, algebraic geometry, differential geometry and quantum logic.

6.12. Our use in $\$ 6.7$ of the traditional notation $\{Y \mid E\}$ for subset formation advertises more than it can deliver on the basis of the monadic hypothesis alone.

Although the symbolic formulation is a little easier to handle than the categorical one, it has to be said that devising nuclei in ASD requires a lot of inspired guesswork. The problem of finding splittings is actually not a new one: it is a feature of Jon Beck's theory of monads that was inherited from his inspiration in homological algebra, where we cannot in general split short exact sequences. This in turn came from the (mathematically interesting) fact that there are non-split extensions of Abelian groups, starting with the cyclic group of order 4.

Like the category of locally compact spaces, $\mathcal{S}$ need not be cartesian closed. However, when we develop induction and recursion in $\$ 9$, we find that the lack of general equalisers is the real handicap to developing the theory. The conjectural extension that we begin to investigate in 12 would provide a much more expressive language in the notation $\{y: Y \mid \cdots\}$, without the need for splittings. It would also provide general equalisers and function spaces.

\section{The Sierpiński space}

In order to put some topological flesh on this categorical skeleton, we need some more specific ideas about the object $\Sigma$. Beware that, in other subjects, $\Sigma$ need not be a two-element set or a subobject classifier ( $c f$.

7.1. We have already made use of the analogy between topology and set theory, in the form of the Lindenbaum-Tarski-Paré theorem in $\$ \$ 4.5 \& 5.3$, as part of the motivation of the monadic property as an abstract formulation of Stone duality. Similarly, we begin by looking at the subobject classifier (lattice of truth-values) $\Omega$ in a topos, in order to identify the relevant properties of its analogue in topology. Its defining property is that any subobject $U \hookrightarrow X$ is the inverse image of 
$\top: \mathbf{1} \hookrightarrow \Omega$ along some unique map $\theta: X \rightarrow \Omega$. That is, there is a pullback

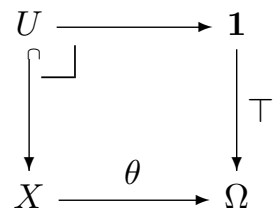

The map $\theta$ is called the classifier, and it is crucial that isomorphic subspaces correspond to equal classifiers.

7.2. Relying on this, if we can show that two constructions yield equivalent subobjects then the corresponding classifying maps are equal. Stripping out all extensional ideas, this means that we regard two propositions $\phi$ and $\theta$ as equal if they are inter-provable. Any proof is as good as any other, in contrast to the view of many type theorists, who regard different proofs of the same proposition as terms of an analogous type ( $c f . \$ 2.2$.

Although individual propositions are not regarded as types in topos theory, $\Omega$ is the type of all propositions. Treating it as an object like any other means that we are doing higher order logic. There are some strange consequences of the translation of inter-provability into equality, since functions of a logical argument must respect equality.

In particular, to assert any $\omega$ (where $\omega \in \Omega$ ) is the same as to say that $\omega$ is equal to $T$, from which it follows that $F \omega=F \top$ for any function $F: \Omega \rightarrow \Omega$. Now, this equality means that $F \omega \Rightarrow F \top$ and $F \omega \Leftarrow F \top$, still under the hypothesis $\omega$. But $\omega$ may be eliminated using Gentzen's introduction rule for $\Rightarrow(c f . \sqrt{2.1})$, in the form

$$
\frac{\Gamma, \omega \vdash \alpha \Rightarrow \beta}{\overline{\Gamma \vdash \omega \wedge \alpha \Rightarrow \beta,}}
$$

giving $\omega \wedge F \omega \Rightarrow F \top$ and $F \omega \Leftarrow \omega \wedge F \top$. Hence we deduce the Euclidean principle,

$$
\text { for any } F: \Omega \rightarrow \Omega \text { and } \omega: \Omega, \quad \omega \wedge F \omega \Longleftrightarrow \omega \wedge F \top \text {. }
$$

We shall see that this is more than just a curiosity of higher order logic.

7.3. In a topos, $\Omega$ classifies arbitrary subobjects, but Giuseppe Rosolini refined the definition to handle restricted classes of monos in other kinds of categories, such as open subspaces in topology and recursively enumerable ones in the theory of computability. The class of monos, which he called a dominion, must include all isomorphisms and be closed under composition and under pullback along arbitrary maps. Then a dominance $\top: \mathbf{1} \rightarrow \Sigma$ has the same definition as $\Omega$, but restricted to the given class of monos Ros86. The Euclidean principle holds for any dominance $\Sigma$ of which all powers $\Sigma^{X}$ exist, not just for $\Omega$ in a topos, as is shown diagrammatically in $[$ ]. We shall prove the converse in 88.4 and recover the topos situation in $\$ 9.3$.

7.4. Now consider the Sierpiński space in classical topology. This has two points, $T$ and $\perp$, of which the former is open and the latter closed, like this:

$$
\begin{array}{ll}
\top: & (\odot) \\
\perp & (
\end{array}
$$

According to our stated methodology, we employ this space because of its universal property. This says that there is a three-way bijective correspondence amongst
(a) open subspaces $U \subset X$,
(b) continuous maps $\theta: X \rightarrow \Sigma$ and
(c) closed subspaces $C \subset X$, 
where we shall say that $\theta$ classifies $U \equiv \theta^{-1}(\top)$ and co-classifies $C \equiv \theta^{-1}(\perp)$. Diagrammatically, these inverse images are given by the two pullbacks

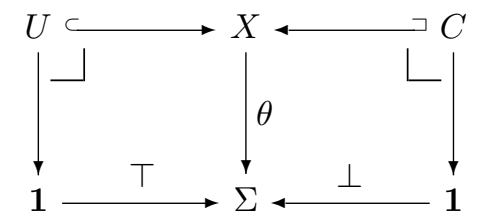

Either of the subobjects $U$ or $C$ determines $\theta$ (and so the other subobject) uniquely, so open inclusions form a dominion for which $T: \mathbf{1} \rightarrow \Sigma$ is a dominance, whilst closed inclusions form another dominion for which $\perp: \mathbf{1} \rightarrow \Sigma$ is a dominance.

7.5. It is very difficult to assess the significance of a classical statement about the two-element set ( $c f . \sqrt{3.4}$ ), so once again we turn to locale theory for a constructive view. An "open subspace" of a locale is an element of the corresponding frame, $a: \mathbf{1} \rightarrow A$, and such elements correspond bijectively to frame homomorphisms $\mathrm{F} \mathbf{1} \rightarrow A$. As our candidate for the Sierpiński locale, we therefore have the free frame F1 on one generator [JT84, §IV 3]; by 4.4 , this is the lattice of Scott-open subsets of $\mathcal{P}(\mathbf{1}) \cong \Omega$.

Recall from 4.8 that the open and closed sublocales of $X$ named by $a \in A$ are given by the nuclei $a \Rightarrow(-)$ and $a \vee(-)$ respectively. If $b \in A$ gives rise to an isomorphic sublocale of either kind then the corresponding nuclei are equal as endofunctions, but by applying them both to $\perp$, $a$ and $b$, we find that $a=b$. So the Sierpiński locale enjoys the same universal property as its classical analogue.

7.6. Hence, in the category of locally compact locales, the Sierpiński locale is a dominance in two ways. It therefore satisfies both the Euclidean principle and its lattice dual.

Besides this, all endofunctions of $\Sigma$ preserve its order. Putting all three of these properties together, we deduce that

$$
F \sigma \Longleftrightarrow F \perp \vee \sigma \wedge F \top
$$

for any $F: \Sigma^{\Sigma}$ and $\sigma: \Sigma$. This equation is called the Phoa principle, and had already been observed in computability theory. Indeed, Martin Hyland, who named this principle after his student Wesley Phoa (pronounced "Pwah"), emphasised that $\Sigma$ should classify both RE and co-RE subsets Hyl91.

7.7. Once again, it is difficult to see the real meaning of such simple formulae as the Euclidean and Phoa principles. The latter says say that any function $F: \Sigma \rightarrow \Sigma$ is defined by a polynomial in one variable and the algebraic operations, $T, \perp, \wedge$ and $\vee$. This means that $\Sigma^{\Sigma}$ (the topology on the Sierpiński space) is the free algebra F1 on one generator, as we found in locale theory. It suggests a generalisation to algebraic or differential geometry, that any endomorphism $F: R \rightarrow R$ of the base ring considered as a space in the new category, is defined by a polynomial in one variable [Koc05, I 12.3].

Another way of viewing this principle may be that it is what is required to make the monad for the "symbolic" algebraic structure (such as $T, \perp, \wedge, \vee$ ) agree with that of the $\lambda$-calculus $\left(\Sigma^{(-)} \dashv \Sigma^{(-)}\right)$.

It was in order to provoke investigation of this analogy that I gave the name Euclidean principle to the earlier equation, since it resembles one step of the Euclidean algorithm for highest common factors. If this really is one case of a general phenomenon, polynomials are the fundamental things, whilst subobjects are a manifestation of them that is peculiar to set theory and topology. 
7.8. In the next section we shall add the Phoa principle to the monadic calculus from $\$ 6$, and prove some results from it that look a lot like the familiar properties of open, closed and compact subspaces. Of course, this indicates that the intuitions that we have considered so far are sound and substantial.

What's surprising is how much can be done in the absence of an axiom that actually states Scott continuity (\$11). One might expect this to be essential for topology, especially considering its fundamental role in the applications in computer science since Scott's original work in the 1970s.

Even though the theory at this second stage is manifestly incomplete, it is worthy of study as it stands, because it is still fully lattice dual. We can rewrite any theorem by interchanging (all of) the following symbols or concepts with their partners, and obtain another valid theorem:

$$
\begin{aligned}
& \leqslant \Rightarrow \top \wedge \exists=\text { open discrete ? open } \\
& \geqslant \Leftarrow \perp \vee \forall \neq \text { closed Hausdorff compact proper }
\end{aligned}
$$

In particular, in 8.5 we shall have a proof rule that looks like Gentzen's for classical $\neg$, alongside the one in $\$ \$ 2.1 \& 7.2$ for $\Rightarrow$. The "classical" rule is valid constructively (in particular, in intuitionistic locale theory, $\$ 7.5$ because it says the same thing for inclusions of closed subspaces that the "intuitionistic" rule says about open ones. Open and closed subspaces are related via their common classifiers $(\$ 7.4)$, and not by set-theoretic complementation. Statements in ASD are therefore free of the double negations that plague mathematics based on intuitionistic set theory, whether that be categorical or type-theoretic.

7.9. Symmetries like this in a theory are powerful because they are predictive. (Consider Mendeleev's periodic table, or Dirac's relativistic quantum mechanics.) When we pair up concepts with their duals, we often find that some things were missing. (New elements or particles, such as the positron.)

What is the counterpart of compactness in the table above? Since the symbolic form of one topological concept is the universal quantifier ( 5.7$)$, the missing idea must be given by the existential quantifier.

Turning to locale theory for more inspiration, a compact locale is one whose terminal projection $K \rightarrow \mathbf{1}$ is proper, so we are looking for those for which this map is open, and indeed they were studied in [JT84, Joh84] and called open locales. Bourbaki spaces throw no light on this property, because they all have it. Casting our philosophical net a little wider, however, we find analogous ideas in computability theory (recursive enumerability) and constructive analysis (locatedness Spi07]). The common theme seems to be an explicit presentation of something.

However, when we look at subspaces that are "open" in this sense, it turns out that they are often (but by no means always) closed subspaces, so we need another word for the idea. In English, the word overt means "open" in its sense of being explicit, so it captures these ideas very well, although it is a little difficult to find appropriate translations into other languages.

Bourbaki spaces and locales treat infinitary unions and finitary intersections asymmetrically. This is explained in ASD by saying that we may form joins indexed by overt objects and meets by compact ones.

\section{Topology using the Phoa principle}

In this section we add the lattice structure on $\Sigma$ and either the Euclidean or the Phoa principle to the monadic framework that we introduced in \$6. The latter plays (part of) the role of the set theory that we take granted when we study other subjects such as group theory, but which we have eschewed for computable topology. So the theory will start to look a little more like an "orthodox" development of theorems from axioms $(\$ 3.2)$. 
See $\mathrm{C}, \mathrm{D}$ for the details of the results in this section, but, despite the lettering, they were written before the monadic $\lambda$-calculus of $\$ 6$ was devised, and expressed in a less clear categorical language. Since the empirical arguments were also not as well developed there as they are were in the previous section, we shall give some of the basic results here in detail. On the other hand, [I, J] give better treatments of some topics that are closer to the applications, especially the theory of compact subspaces.

8.1. In addition to the axioms in 86.1 , we now require the type $\Sigma$ to have

(a) operations $\top, \perp: \mathbf{1} \rightrightarrows \Sigma$ (in place of $\star, 6.1(\mathrm{~b})$ and $\wedge, \vee: \Sigma \times \Sigma \rightrightarrows \Sigma$ that satisfy

(b) the equations for a distributive lattice; and

(c) the Euclidean principle $\sigma \wedge F \sigma \Leftrightarrow \sigma \wedge F \top$ if we want to consider set theory; or

(d) the Phoa principle $F \sigma \Leftrightarrow F \perp \vee \sigma \wedge F \top$ for general topology;

where $\sigma: \Sigma$ and $F: \Sigma^{\Sigma}$.

8.2. The lattice structure defines an order relation on $\Sigma$ and its powers $\Sigma^{X}$. Following logical custom, we have already started to write $\Rightarrow$ and $\Leftrightarrow$ for the order and equality on $\Sigma$. We retain $\leqslant$ and $=$ for these relations on its powers (the symbols $\subset$ and $\subseteq$ are inappropriate, for many reasons), but application to an argument changes them into $\Rightarrow$ and $\Leftrightarrow$, so if $\phi \leqslant \psi$ then $\phi a \Rightarrow \phi a$, whilst if $\phi=\psi$ then $\phi a \Leftrightarrow \phi a$.

In the topological case $(\mathrm{d})$, all $\mathcal{S}$-morphisms $\Sigma^{Y} \rightarrow \Sigma^{X}$ preserve $\leqslant$, so $\mathcal{S}$ is an ordered category, which is a special case of a 2-category. In particular, we may talk about adjoint maps $\Sigma^{Y} \leftrightarrows \Sigma^{X}$ with respect to this order: $L \dashv R$ means that id $\leqslant R \cdot L$ and $L \cdot R \leqslant$ id. This is also possible in the set-theoretic case (c), so long as we're careful, as logical negation $(\neg)$ reverses the order.

We may extend $\leqslant$ to an order on any object $X$, where

$$
x \sqsubseteq_{X} y \text { means }(\lambda \phi . \phi x) \leqslant(\lambda \phi . \phi y) .
$$

By sobriety ( 6.3 ) and monotonicity, $\sqsubseteq$ is antisymmetric and (when $X \equiv \Sigma^{Y}$ ) agrees with $\leqslant$. Wesley Phoa introduced his principle [Pho90] to make this order equivalent to the existence of a link map $\ell: \Sigma \rightarrow X$ with $\ell \perp=x$ and $\ell \top=y$, which he used to construct limits of the objects that he was considering.

8.3. The Euclidean principle is a purely algebraic property, at least if you are willing to regard $\lambda$-calculus as algebra. However, in the context of the monadic framework, it automatically yields the higher order structure of a dominance $(7.3)$, classifying certain subobjects. We shall build up to a characterisation of elementary toposes in $\$ 9.3$

First we have to show that the pullback in \$7.1 exists. It is equivalent to the equaliser

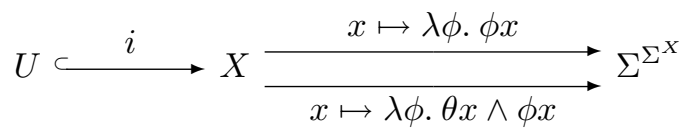

that is an example of the one in 86.5 , with $E \equiv \lambda \phi . \theta \wedge \phi$. Notice that this is the same formula that we saw in $\$ 5.6$ when we showed that open subspaces are $\Sigma$-split.

The question is therefore whether $E$ is a nucleus; expanding the $\lambda$-calculus definition in 6.7 . we require that

$$
\mathcal{F}(\lambda \psi . \psi y \wedge \theta y) \wedge \theta y \Longleftrightarrow \mathcal{F}(\lambda \psi \cdot \psi y) \wedge \theta y .
$$

This follows from the Euclidean principle, putting $\sigma \equiv \theta y$ and $F \equiv \lambda \tau . \mathcal{F}(\lambda \psi \cdot \psi y \wedge \tau)$. Conversely, considering $Y \equiv \mathbf{1}, \theta \equiv \lambda y . \sigma$ and $\mathcal{F} \equiv \lambda G . F(G \top)$, it is also necessary.

Hence the Euclidean principle is exactly what we need to satisfy the abstract definition of nucleus. On the other hand, $E$ satisfies the more user-friendly lattice-theoretic characterisation of 
nuclei that we also mentioned in 6.7 , just using the distributive law. Of course, that is because the proof of this characterisation makes use of the Phoa principle $(\$ 11.6)$.

By a similar argument, $\lambda \phi . \phi \vee \theta$ is a nucleus (for the closed subspace coclassified by $\theta$ ) iff $\Sigma$ satisfies the dual Euclidean principle.

8.4. We may therefore use the monadic framework in $\$ 6$ to define the pullbacks in $\$ 7.1$ and $\$ 7.4$ Then, bearing in mind that we shall have to verify the stronger meanings that these words have outside this paragraph, we call an inclusion $i: U \hookrightarrow X$ open if it (is isomorphic to one that) is the pullback of $\top: \mathbf{1} \rightarrow \Sigma$ along from some (not $\hat{a}$ priori unique) $\theta: X \rightarrow \Sigma$, which classifies $U$. In particular, $\theta \equiv \top$ classifies id : $X \hookrightarrow X$.

In order to prove that open inclusions form a dominion with dominance $\Sigma$ in the sense of Rosolini ( $(7.3)$, we also need to show that the composite of two open inclusions is open. For this, we use the $\Sigma$-splitting $I$ that is provided by the monadic framework.

Suppose that $V \stackrel{j}{\hookrightarrow} U \stackrel{i}{\hookrightarrow} X$ are classified by $\phi: \Sigma^{U}$ and $\theta: \Sigma^{X}$ respectively. Since $I$ splits $\Sigma^{i}$, we have $\phi u \Leftrightarrow \Sigma^{i}(I \phi) u \Leftrightarrow(I \phi)(i u)$, so the bottom right square commutes.

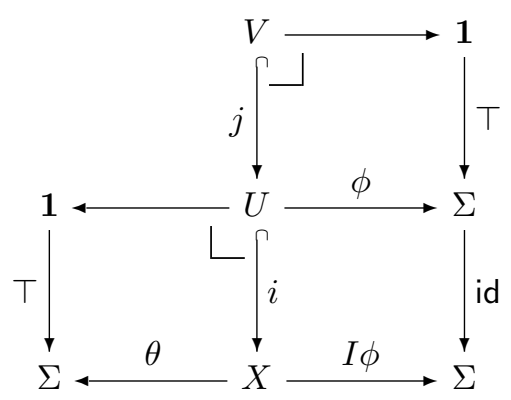

We claim that the rectangle on the right is a pullback, i.e. $I \phi$ classifies $V \subset X$. First, note that $I \phi=\left(I \cdot \Sigma^{i} \cdot I\right) \phi=E(I \phi)=\theta \wedge I \phi \leqslant \theta$. If $x: \Gamma \rightarrow X$ and $\Gamma \rightarrow \mathbf{1}$ make a commuting quadrilateral then $\top \Leftrightarrow I \phi x \Rightarrow \theta x$. So $x=i u$ since $\theta$ classifies $U$, and $\phi u \Leftrightarrow(I \phi)(i u) \Leftrightarrow I \phi x \Leftrightarrow \top$, so $u=j v$ since $\phi$ classifies $V$. Hence $x=(i \cdot j) v$, and $v$ is unique since $i \cdot j$ is mono.

If $\psi \leqslant \theta$ then $\psi=\psi \wedge \theta=E \psi=I \phi$ where $\phi \equiv \Sigma^{i} \psi$. So the open inclusion $W \hookrightarrow X$ classified by $\psi$ factors through $U \hookrightarrow X$ classified by $\theta$ iff $\psi \leqslant \theta$ iff $\psi=I \phi$ for some $\phi: \Sigma^{U}$, and then $\phi$ classifies $W \hookrightarrow U$. Hence $U \cong W$ iff $\theta=\psi$, so classifiers are unique.

We are justified in calling $i$ an "open" inclusion because $I \dashv \Sigma^{i}$ in the sense of $₫ 8.2$, and the Frobenius and Beck-Chevalley laws $(2.9$ hold [C, Prop. 3.11]. If we want to use this result in the study of set theory $(\delta 8.1(\mathrm{c})]$ then we must be careful not to assume that $I$ preserves $\leqslant$, but the same result also proves this. We write $\exists_{i}$ for $I$, for reasons that we explain in 8.7 .

By reversing the order $\leqslant$ throughout this argument and assuming the dual Euclidean principle, $\perp: \mathbf{1} \rightarrow \Sigma$ is also a dominance. We call the inclusions that are expressible as pullbacks of $\perp$ along $\theta: \mathbf{1} \rightarrow \Sigma$ closed, say that $\theta$ co-classifies them and write $\Theta_{i}$ for $I$. Beware, however, that if $\theta \leqslant \phi$ classify $C$ and $D$ respectively then $D \subset C$.

$8.10(\mathrm{~g})$ gives an important converse result.

8.5. In practice, it is often easier to reason with the Gentzen-style rules

$$
\frac{\Gamma, \sigma \Leftrightarrow \top \vdash \alpha \Rightarrow \beta}{\Gamma \vdash \sigma \wedge \alpha \Rightarrow \beta} \quad \frac{\Gamma, \sigma \Leftrightarrow \perp \vdash \alpha \Rightarrow \beta}{\Gamma \vdash \alpha \Rightarrow \beta \vee \sigma}
$$

that are equivalent to the Euclidean principle and its dual, $c f . \$ \$ 2.1,7.2 \& 7.8$

To do this, we first have to modify the definition of contexts in $\$ 6.1$, which now consist of both typed variables and equational hypotheses of the forms $\sigma \Leftrightarrow \top$ and $\sigma \Leftrightarrow \perp$ for $\sigma: \Sigma$. We proved 
the Euclidean principle from the Gentzen rule on the left using such contexts in $\$ 7.2$, and the dual is similar.

This modification is syntactic sugar. A context consisting of several variables and hypotheses of both kinds may be interpreted as a locally closed subspace (i.e. the intersection of a closed subspace with an open one) of the product of the types of the variables.

Using the Euclidean principle, the open subspace classified by $\sigma$ is $\Sigma$-split with nucleus $E \equiv$ $\lambda \phi . \sigma \wedge \phi$. So the inequality $\alpha \Rightarrow \beta$ between subsubspaces is defined as $\alpha \wedge \sigma \Rightarrow \beta \wedge \sigma$ in the ambient space, which is what the Gentzen rule says.

These rules do not force $F: \Sigma^{\Sigma}$ to preserve the order - this has to be stated separately. Beware that we use this property so often in topology that we usually take it as read.

8.6. Now we can ask when particular subspaces are open or closed, starting with the diagonal $X \subset X \times X$. It is natural to call $X$

(a) discrete if the diagonal is open, its classifier being called equality, $=_{X}$; and

(b) Hausdorff if the diagonal is closed, its co-classifier being called inequality or apartness, $\neq_{X}$ or $\# x$.

For example, we expect $\mathbb{N}$ and $\mathbb{Q}$ to have both properties $(\sqrt{9.5})$, and $\mathbb{R}$ to be Hausdorff but not discrete $(11.10)$. This is in line with the computational fact that we may decide equality of integers or rationals, but only detect when real numbers are different.

Beware that, whilst points and the diagonal are open in a discrete space, arbitrary subspaces need not be. This is because the computable topology on the discrete space $\mathbb{N}$ consists of the recursively enumerable subsets, not all of them. Also, our definition of Hausdorffness is slightly different from that in locale theory [Sim78, Joh82, §III 1.3].

Classically, any discrete space is Hausdorff, but the proof employs a union that is illegitimate for us, and any free algebra with insoluble word problem provides a counterexample. Terms are equal iff they are provably so from the equations, so we observe equality by enumerating proofs, but none need be forthcoming. In particular, combinatory algebra (with a non-associative binary operation and constants $k$ and $s$ such that $(k x) y=x$ and $((s x) y) z=(x z)(y z))$ encodes the untyped $\lambda$-calculus, and therefore arbitrary computation, so equality is undecidable.

We use a subscript or brackets, $n={ }_{X} m$ or $(n=m)$, to indicate the term of type $\Sigma$ that this definition provides in a discrete space, with computable equality. This convention avoids ambiguity of notation with the underlying term calculus, in which any two terms of the same type may be provably equal or not.

The proof rules that relate these two notions of (in)equality are

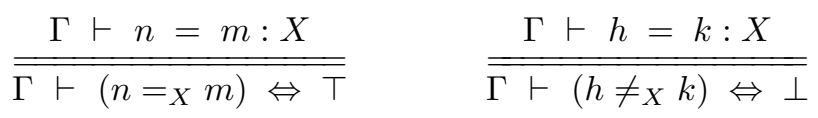

The rule for a Hausdorff space is not doubly negated: this is simply the way in which we express membership of a closed subspace, whilst $\neq$ is the natural name for its co-classifier.

From the Gentzen rules in 8.5 we obtain respectively

$$
\phi x \wedge\left(x==_{X} y\right) \Rightarrow \phi y \quad \text { and } \quad \phi x \vee\left(x \neq_{X} y\right) \Leftarrow \phi y .
$$

The usual properties of equality (reflexivity, symmetry, transitivity and substitution) follow from the first of these, whilst the dual argument gives the analogous properties of inequality. See J, $\S \S 4-5]$ for more detail in the setting of applications. 
8.7. Turning to compactness, recall from $\$ 5.7$ that it can be expressed using an operator $A: \Sigma^{\Sigma^{X}}$. This is Scott continuous in the model (locally compact Bourbaki spaces), but is simply a term or morphism in the axiomatisation.

It is enough to define a compact object $K$ as one for which the map $\Sigma \rightarrow \Sigma^{K}$ by $\sigma \mapsto \lambda k . \sigma$ has a right adjoint, $\forall_{K}$, in the sense of $\$ 8.2$. The two directions of the adjoint correspondence are exactly the introduction and elimination rules of the symbolic formulation, in which it's more convenient to write $\forall k: K . \phi k$ than $\Theta_{K}(\lambda k: K . \phi k)$.

However, in topology, we also have the dual Frobenius law (cf. 2.9 ,

$$
\forall k .(\sigma \vee \phi k) \Longleftrightarrow \sigma \vee \forall k . \phi k,
$$

for free, from the Phoa (or dual Euclidean) principle $(88.1(\mathrm{~d})$, with $F \equiv \lambda \tau . \forall k . \tau \vee \phi k$. This does not hold in intuitionistic set theory, but it was identified in intuitionistic locale theory by Japie Vermeulen Ver94.

The first of the familiar properties of compact spaces is that any closed subspace $i: C \subset K$ is also compact. Its quantifier is given by $\uplus_{C} \equiv \forall_{K} \cdot \boldsymbol{U}_{i}$, where $\boldsymbol{U}_{i}$ is the $\Sigma$-splitting of $i$ in $\S \$ 5.6$ \& 8.4 [C, Prop. 8.3].

Products and coproducts of compact spaces are again compact, as are equalisers and pullbacks targeted at Hausdorff spaces [C, $\S \S 8-9]$.

The Beck-Chevalley condition (\$2.9) also comes for free, as substitution under $\lambda$ [C, Prop. 8.2]. Its lattice-theoretic interpretation is that any topology $\Sigma^{X}$ admits $K$-indexed meets, and not just finite ones $(\$ 7.9)$.

8.8. When we try to apply these ideas to compact subspaces, we find that the monadic framework in $\$ 6$ does not provide a sufficiently general theory (so it doesn't extend to proper maps either). This is related to the fact that, in the leading model $(\$ 5.4)$, a compact subspace of a non-Hausdorff locally compact Bourbaki space need not itself be be locally compact. This is discussed in [G, §5], which is based on HM81.

The clearest treatment for ASD is that in [J, §8], which exploits the fact that the main object of study in that paper is a Hausdorff space $(\mathbb{R})$, by restricting attention to subspaces that are both closed and compact. Since a Hausdorff space $H$ has $\neq$, we expect the operator $A: \Sigma^{\Sigma^{H}}$ to represent (as a compact space) the closed subspace that is co-classified by

$$
\theta \equiv \lambda x . A(\lambda y . x \neq y) \quad \text { if } \quad \frac{\phi \vee \theta=\top}{\overline{A \phi \Leftrightarrow \top}} \quad \text { for any } \phi: \Sigma^{H} .
$$

If $H$ is also compact, we obtain conversely

$$
A \phi \Longleftrightarrow \forall x: H . \omega x \vee \phi x .
$$

Hence closed and compact subspaces coincide in a compact Hausdorff space, because there

$$
\phi x \Longleftrightarrow \forall y: H .(x \neq y) \vee \phi y
$$

which is obtained from the formula in $\$ 8.6$. Notice that $A$, like $\theta(\$ 8.4)$, decreases in the order $(\$ 8.2)$ as the compact or closed subspaces get bigger. 
8.9. To make sense of this equivalence between closed and compact subspaces, however, we need some way of saying that $K$ is "the same subspace" as $C$. We do this by defining

$$
a \in C \quad \text { as } \quad \theta a \Leftrightarrow \perp \quad \text { and } \quad a \in K \quad \text { as } \quad A \leqslant \lambda \phi . \phi a
$$

for any term $a: X$, possibly including parameters.

The definition of $a \in K$ is motivated by the idea in Wil70, HM81 that a compact subspace is the intersection of its open neighbourhoods. In a non-Hausdorff space, however, this intersection may be larger than the original compact subspace, and is called its saturation. Nevertheless, $C$ and $K$ do have the same "elements" according to the definitions that we have just given. The account in [J, §8] also treats direct images and compact open subspaces.

8.10. Because of the lattice duality of the axioms that we have introduced so far ( $\S ₫ 7.8$ ) , there is a dual notion to compactness. We call it overtness, and it is related to the existential quantifier.

By the same arguments as for compact Hausdorff spaces [C, $\S \S 6-9]$,

(a) $\mathbf{0}$ and $\mathbf{1}$ are overt, as are $X \times Y$ and $X+Y$ if $X$ and $Y$ are;

(b) any open subspace of an overt space is again overt;

(c) any equaliser or pullback of overt spaces targeted at a discrete space is overt;

(d) any overt subspace of a discrete space is an open subspace;

(e) any direct image of an overt space is overt;

(f) any map from an overt object to a discrete one is an open map; and

(g) any mono $f: X \longmapsto Y$ from an overt object to a discrete one is an open inclusion, classified by $\theta \equiv \lambda y \cdot \exists x .\left(f x==_{Y} y\right)$

See [C, $\S \S 8$ \& 10] for the proofs, which were inspired by similar results in [JT84. We spell out the last part, as it will be important later. Define

$$
F: \Sigma^{X} \rightarrow \Sigma^{Y} \quad \text { by } \quad F \phi \equiv \lambda y: Y . \exists x: X . \phi x \wedge\left(f x==_{Y} y\right) .
$$

Then

$$
F \phi(f x) \Longleftrightarrow \exists x^{\prime}: X . \phi x^{\prime} \wedge\left(f x^{\prime}=_{Y} f x\right) \Longleftrightarrow \exists x^{\prime}: X . \phi x^{\prime} \wedge\left(x^{\prime}=_{X} x\right) \Longleftrightarrow \phi x
$$

by 8 8.6. Hence $f: X \longmapsto Y$ is $\Sigma$-split, and $X \cong\{Y \mid E\}$ by $\S 6.8$, where $E \equiv \Sigma^{f} \cdot F$. Expanding $E$, we find that $X \subset Y$ is the open subspace classified by $\theta(8.3)$.

8.11. We have made a lot of use of the analogy between (Stone duality for) set theory and topology. This is no longer applicable in the case of partial maps, where the Phoa principle makes the topological account much simpler than the set-theoretic one [D].

In Rosolini's theory of dominions and dominances $(87.3)$, a partial map $(i, f): X \rightarrow Y$ consists of an "open mono" $i: U \hookrightarrow X$ and an (ordinary or "total") map $f: U \rightarrow Y$.

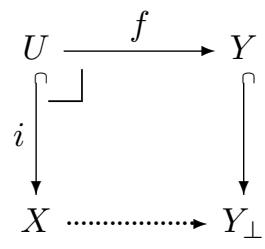

Then $Y \hookrightarrow Y_{\perp}$ is a partial map classifier or lift if, for every partial map $X \rightarrow Y$, there is a unique total map $X \rightarrow Y_{\perp}$ that makes the square a pullback. In particular, $\mathbf{0}_{\perp}=\mathbf{1}, \mathbf{1}_{\perp}=\Sigma$ and $\Sigma_{\perp}=\Sigma^{\Sigma}$, but this coincidence ends there.

Classically, as the notation suggests, $Y_{\perp}$ is obtained by adding a single point $\perp$ to $Y$, to serve as the "undefined" value of $f x$ for $x \notin U$. In topology, when $U \subset X$ is Scott open, and in particular 
upper, $\perp$ must lie below $Y$ in the order. In intuitionistic set theory, on the other hand, we need higher order logic to define $Y_{\perp} \subset \mathcal{P}(Y)$ as "the set of subsets with at most one element".

Using the monadic framework and the Phoa principle, we obtain $Y_{\perp}$ in a much simpler way via its topology. This forms a comma square,

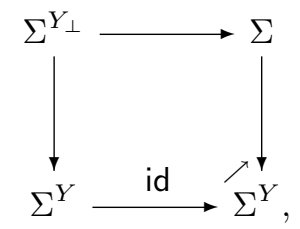

which is defined like a pullback except that the order $\leqslant$ replaces equality in the bottom right. So

$$
\Sigma^{Y_{\perp}} \equiv\left\{\left(\psi: \Sigma^{Y}, \sigma: \Sigma\right) \mid \sigma \Rightarrow \psi y\right\}
$$

in set-theoretic notation. Since this is a retract of $\Sigma^{Y} \times \Sigma$, we may also construct the comma square in this way in ASD.

We also need to define the Eilenberg-Moore algebra structure on $\Sigma^{Y_{\perp}}$ and prove the universal property of $Y_{\perp}$. The latter involves maps $X \rightarrow Y_{\perp}$, which are homomorphisms out of $\Sigma^{Y_{\perp}}$, whereas the definition of a comma square uses incoming maps. It's not difficult to prove the result using Scott continuity, but without this it's rather delicate. The key idea is the isomorphism

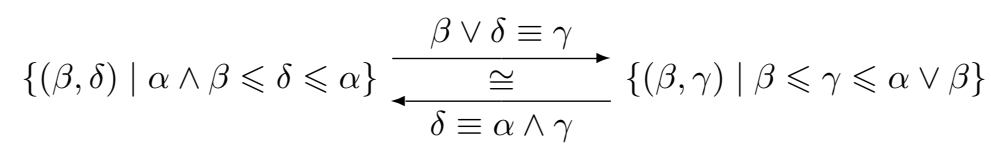

that follows from distributivity, but is actually a weaker property called modularity that holds in many lattices of subalgebras, such as those of groups and vector spaces.

8.12. This comma square construction is a special case of Artin gluing, which recovers the topology of a space from those of an open subspace and its closed complement, together with a map that encodes the way in which these fit together AGV64, Exp. IV §9.5].

However, the general version of Michael Artin's construction does not work in ASD, because it is computable topology and so inherits some of the strange behaviour of computability theory. In particular, the open (RE) subspace of $\mathbb{N}$ consisting of codes of terminating programs does not glue to its closed (co-RE) complement in anything like the Artin fashion [D, §4].

The special case of the lift is also known is topology as the scone (Sierpiński cone) and in topos theory as gluing or the Freyd cover. See [Tay99, $\S \S 7.7 \& 9.4]$ for further mathematical and historical discussion.

\section{Discrete mathematics}

Although we began by rejecting set theory as foundations, we still need some account of "sets" as discrete spaces for combinatorial purposes, especially in algebraic topology, and to study computation itself. Even within general topology, sets of some kind are needed to index the families of open and compact subspaces in a locally compact space $(\$ \$ 5.8 \mathrm{ff})$, where the discrete name is needed because the terms that encode the subspaces in the pair vary in opposite directions $(\S \Phi 8.4$ \& 8.8) with respect to the intrinsic order $(8.2$ [ $\mathrm{G}$.

We do not expect our "computable set theory" to be anywhere near as powerful as the traditional one ( $c f . \$ 10$ ), but category theory provides suitable weaker alternatives. However, our monadic framework will need to be modified in order to reason by induction. 
Since the importance of the results about ASD that we describe in this section only emerged gradually and piecemeal, they come from throughout its development. On a larger scale, this is of course also true of the history of symbolic and categorical logic.

9.1. Let's look first at the properties that a category of "discrete" spaces might have in a computable theory. A pretopos is a category $\mathcal{E}$ that has

(a) finite products $(\mathbf{1}$ and $\times)$;

(b) equalisers and pullbacks;

(c) an initial object $\mathbf{0}$ that is strict, i.e. any $N \rightarrow \mathbf{0}$ is an isomorphism;

(d) finite coproducts $(+)$ that are disjoint, and stable under pullback;

(e) quotients of equivalence relations that are stable under pullback, and effective in the sense that the kernel of the quotient is the given equivalence relation.

Finite limits and stable effective quotients of equivalence relations were studied in category theory long before it considered logic, because categories of finitary algebras inherit them from sets [BGvO71. As the name suggests, pretoposes capture (the finitary) part of the notion of (Grothendieck) topos, in a characterisation due to Jean Giraud. Gir72. This included stable disjoint coproducts, but they were later reformulated in a new way called extensivity Coc93, CLW93. This is very useful, because it is often technically easier to check that it holds in many categories of "spaces", understood in the very general sense of 4.2 Several textbooks include accounts of pretoposes, including [Tay99, Ch. V].

9.2. The sense in which we use the word "discrete" in ASD, namely having equality (88.6), is too weak for this purpose. We shall take overt discrete objects as our "sets" i.e. those that also have $\exists(8.10)$, and we adopt the convention of using $N$ and $M$ for these objects, since they have the topological properties of $\mathbb{N}$.

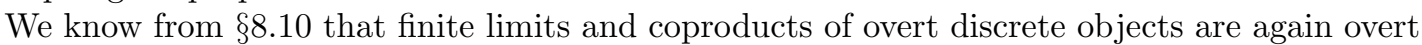
discrete. But they also admit stable effective quotients of open equivalence relations [C, §10]. To prove this, we have to construct the topology of the quotient, $\Sigma^{N / R}$, as a $\Sigma$-split subspace of $\Sigma^{N}$. Then $N / R$ itself is a $\Sigma$-split subspace of $\Sigma^{2} N$, given by the nucleus $\lambda \mathcal{F} F . F(\lambda x . \mathcal{F}(\lambda \phi . \exists y \cdot R(x, y) \wedge$ $\phi y))$ [B, Ex. 11.13].

Overt discrete spaces therefore form a pretopos, as do compact Hausdorff spaces, since we still have lattice duality. It was because of this remarkable result that I decided in 1997 to devote my entire research effort to ASD.

9.3. In the extreme case, every object of the category $\mathcal{S}$ is overt discrete. This brings us back to the Lindenbaum-Tarski-Paré theorem $(\S \S 4.5 \& 5.3)$, as we now have enough structure to complete the characterisation of set theory (2.10). Any category $\mathcal{S}$ is an elementary topos iff it satisfies

(a) the axioms for the monadic framework in $\$ 6.1$.

(b) the Euclidean principle $88.1(\mathrm{c})$ and

(c) every object is overt and discrete.

Since every map goes from an overt object to a discrete one, every mono is an open inclusion $(88.10(\mathrm{~g})]$, i.e. classified by an element of $\Sigma$. Also, discreteness of $\Sigma$ makes it into a Heyting algebra, where $\sigma \Rightarrow \tau$ is $\sigma=_{\Omega}(\sigma \wedge \tau)$ [], §11].

This does not, however, describe the computational situation, in which there can be no operation like $\neg, \Rightarrow$ or $=_{\Sigma}$, because it would solve the halting problem [Tur35. For the same reason, the subspace of $\mathbb{N}$ consisting of codes for non-terminating programs is not overt. 
9.4. The main concerns in the foundations of discrete mathematics are of course the natural numbers, induction and recursion. The numerals themselves emerge from the introduction rules: zero and the successor operation. The elimination rule,

$$
\frac{\Gamma \vdash z: X \quad \Gamma, n: \mathbb{N}, x: X \vdash s(n, x): X}{\Gamma, n: \mathbb{N} \vdash \operatorname{rec}(n, z, s): X}
$$

for which the $\eta$-rule is $\operatorname{rec}(n, 0,+1)=n$ and the $\beta$-rules are

$$
\operatorname{rec}(0, z, s)=z \quad \text { and } \quad \operatorname{rec}(n+1, z, s)=s(n, \operatorname{rec}(n, z, s)),
$$

defines primitive recursion at type $X$. We sometimes specify the type when it is necessary to restrict its generality, because some logical investigations become too difficult for arbitrary types. In symbolic logic, the defining expressions $z$ and $s$ are naturally understood to allow parameters, which it is the role of the context $\Gamma$ to manage $(\$ 2.2$.

Bill Lawvere captured these ideas categorically by observing that $(\mathbb{N}, 0,+1)$ is the free algebra for the endofunctor $\mathbf{1}+(-)$; then the universal property compares it with another algebra $(X, z, s)$. However, as with $\vee$ and $\exists(\S 2.5(\mathrm{e}))$, this does not make allowance for parameters. One way to handle them is by using recursion at type $X^{\Gamma}$, if we are working in a cartesian closed category, but our category $\mathcal{S}$ does not have general exponentials like this, so we shall keep the explicit parameters.

9.5. Many familiar things can be defined by recursion on $\mathbb{N}$, not least its arithmetic, but we just note those that we shall need later in this paper. In particular, we may define a map cmp : $\mathbb{N} \times \mathbb{N} \rightarrow \mathbb{N}$ using the equations

$$
\begin{array}{rlrl}
\mathrm{cmp}(0,0) & =1 & \mathrm{cmp}(n+1,0) & =2 \\
\mathrm{cmp}(0, m+1) & =0 & \mathrm{cmp}(n+1, m+1) & =\mathrm{cmp}(n, m),
\end{array}
$$

whilst $\phi(0)=\perp$ and $\phi(n+1)=\top$ give one $\phi: \mathbb{N} \rightarrow \Sigma$. From these we derive the six relations $=, \neq,<,>, \leq$ and $\geq$ and their usual properties as terms of type $\Sigma$ on $\mathbb{N}$, which is therefore both discrete and Hausdorff.

Notice that we distinguish the symbol $\leq$ for the arithmetical order from the logical order $\leqslant$ that is defined on $\Sigma^{X}(88.2$. Then $\leq$ is imposed structure, because functions $\mathbb{N} \rightarrow \mathbb{N}$ do not have to preserve it, whereas $\Rightarrow, \leqslant$ and $\sqsubseteq$ are intrinsic, in that every morphism necessarily preserves them.

Hence, for each numeral $n$, the object $\mathbf{n} \equiv\{m: \mathbb{N} \mid m<n\}$ may be defined as a subspace of $\mathbb{N}$ that is both open and closed. It is also both overt and compact, with quantifiers that are definable from $\vee$ and $\wedge$ by primitive recursion.

9.6. There are tricks for encoding pairs and lists as integers, but they are computationally expensive, whilst logic and functional programmers have long known a better way of doing things. A simple modification of the definition of $\mathbb{N}$ yields a very useful and general data structure, $\mathbb{T}$, of binary trees. Instead of the unary successor operation for $\mathbb{N}, \mathbb{T}$ has pairing, which is written $[a \mid b]$, whilst the projections are called car and cdr in memory of some ancient hardware [McC78]. This is also of theoretical utility, because $\operatorname{List}(\mathbb{T}) \cong \mathbb{T}$, where

$$
[a, b, c, \ldots, z] \equiv[a \mid[b \mid[c \mid[\ldots \mid[z \mid \mathbf{0}] \ldots]]]] .
$$


9.7. Combining $\mathbb{N}$ or $\mathbb{T}$ with the structure of a pretopos gives a very expressive setting, in which we may do anything we like of a combinatorial nature, i.e. definable using explicit enumerations.

In particular, the quotient of a finite object $\mathbf{n}$ by an equivalence relation is finitely generated but not necessarily finitely enumerated, i.e. it can be listed, but possibly with repetitions Tay99. $\S 6.5]$. This weaker notion of finiteness is due to Kazimierz Kuratowski [Kur20. It is more relevant to logic, because we may regard the free semilattice on a set as its collection of Kuratowski-finite subsets [Mik76, Appx 2], so we write $\mathrm{K}(N)$ for what is elsewhere called the finite powerset.

These are the properties that are taught to first year mathematics and computer science undergraduates as naïve set theory or discrete mathematics.

In category theory, an arithmetic universe is a pretopos in which any object $N$ generates a free monoid List $(N)$, from which we may derive $\mathrm{K}(N)$ and other free algebras. This notion was introduced by André Joyal in 1973, as a way of formulating Gödel's incompleteness theorem [Göd31] in a categorical way, but unfortunately he has never published his results. Maria-Emilia Maietti has given a symbolic calculus for arithmetic universes in the style of Martin-Löf [Mai03, Mai05]. One symptom of the paucity of literature on this important topic is that some disagreement remains about the precise details of the definition, in contrast to the more rigorous attention that was

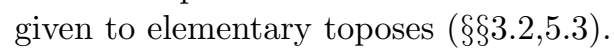

9.8. It is all very well constructing terms by recursion, but we also want to prove properties of them by induction. Since recursion may be used within terms, and not just on the outside, induction does not follow directly from the rules above, and is treated separately in symbolic logic:

$$
\frac{\Gamma \vdash f 0=g 0: X \quad \Gamma, n: \mathbb{N}, f n=g n: X \vdash f(n+1)=g(n+1): X}{\Gamma, n: \mathbb{N} \vdash f n=g n: X}
$$

Notice that, in order to prove some equation $f n=g n: X$ by induction, we need to be able to state it as an induction hypothesis [E, §2].

Categorically, induction can be treated as a special case of recursion, but only when we have the additional structure of the equaliser type:

$$
\Gamma \times \mathbb{N} \ldots \ldots \ldots \ldots \ldots+\left\{\begin{array}{l}
\longrightarrow \\
g
\end{array} X\right.
$$

As is common with universal properties, we use them twice: once to obtain the dotted map, and again to say that the composite $\Gamma \times \mathbb{N} \rightarrow \Gamma \times \mathbb{N}$ has to be the identity, which we obtain symbolically from the $\eta$-rule. It follows that the inclusion into $\Gamma \times \mathbb{N}$ is the whole thing.

9.9. Notice that these equalisers or equational hypotheses have arisen for logical reasons, so I believe that we probably ought to understand them proof-theoretically $(\$ 12)$. For the time being, however, we may give them a meaning amongst either Bourbaki spaces or locales, in either of which we have

(a) the whole category, $\mathcal{L}$, which has all equalisers, but not $\Sigma^{\Gamma}$ for all $\Gamma \in \mathcal{L}(\$ 5.4)$, and

(b) the full subcategory $\mathcal{S}$ of locally compact objects, which has $\Sigma^{X}$ for all $X \in \mathcal{S}$, but not all equalisers. 
9.10. The abstract theory needs to reproduce this situation. Plainly $\mathbb{N}$ should be a "set" in the sense of $\$ 9.2$, so we must also say that it admits existential quantification. Besides the axioms in $\S \S 6.1 \& 8.1$ we therefore require

(a) another category $\mathcal{L}$ that has all finite limits, in which

(b) $\mathcal{S} \subset \mathcal{L}$ is a full subcategory,

(c) the universal properties of $\mathbf{1}, \times$ and $\Sigma^{(-)}$hold in $\mathcal{S}$ for test objects $\Gamma \in \mathcal{L}$,

(d) there is a natural numbers object $\mathbb{N} \in \mathcal{S} \subset \mathcal{L}$ that has

(e) existential quantification, $\exists_{\mathbb{N}}: \Sigma^{\mathbb{N}} \rightarrow \Sigma$, so it is overt $(\$ 8.10$, and

(f) primitive recursion at all objects of $\mathcal{L}$, with parameters from $\mathcal{L}$ too.

In the symbolic language, we distinguish between types (corresponding to objects $X \in \mathcal{S}$ ) and contexts $(\Gamma \in \mathcal{L})$. Any type $X$ has a topology $\Sigma^{X}$, whilst a context $\Gamma$ may include equational hypotheses, so this extension subsumes the one that we made in 8.4 .

The existential quantifier $\exists_{\mathbb{N}}$ breaks the open-closed duality in topology, since we do not equip $\mathbb{N}$ with a universal quantifier too. Topologically, this is because $\mathbb{N}$ is not compact [C, 7.11], whilst computationally it is not possible to observe an infinite conjunction in the way that it is a disjunction.

9.11. Whilst $\mathbb{N}$ exposes one flaw in the monadic framework, in another way it shows its conceptual strength. We call a predicate $\Gamma \vdash \phi: \Sigma^{\mathbb{N}}$ a description if it ought to be "uniquely satisfied" in the sense that

$$
\Gamma \vdash \top \Leftrightarrow \exists n . \phi n \quad \text { and } \quad \Gamma, n, m: \mathbb{N} \vdash \phi n \wedge \phi m \Rightarrow n=m: \mathbb{N} \text {. }
$$

From sobriety of $\mathbb{N}(6.3)$ we deduce that $\phi$ actually has a witness (definition by description), namely

$$
\text { the } n . \phi n \equiv \operatorname{focus}_{\mathbb{N}}(\lambda \psi \cdot \exists m . \phi m \wedge \psi m) .
$$

It is easy to show that $P \equiv \lambda \psi \cdot \exists m . \phi m \wedge \psi m$ satisfies the lattice-theoretic characterisation of primality, but use of that depends on Scott continuity $(\$ 11.6)$. Deducing the $\lambda$-calculus definition from primitive recursion is a little trickier [A, Prop. 10.8].

Conversely, any prime $P: \Sigma^{2} \mathbb{N}$ gives a description (easily [A, Prop. 10.4]) by

$$
\phi n \equiv P(\lambda m . m=n) .
$$

The defining conditions for a description and its operator, "the", provide the introduction rule for another symbolic calculus. The elimination rule says that

$$
\psi(\text { the } n . \phi n) \Longleftrightarrow \exists n . \phi n \wedge \psi n,
$$

the $\beta$-rules justify the name "the $n . \phi n$ ",

$$
\top \Leftrightarrow \phi(\text { the } n . \phi n) \quad \text { and } \quad \phi m \Rightarrow m={ }_{\mathbb{N}} \text { the } n . \phi n,
$$

and the $\eta$-rule says that, for any $n: \mathbb{N}$, the predicate $\phi m \equiv\left(n=_{\mathbb{N}} m\right)$ is a description, with $n=$ the $m . \phi m$. Like focus, the description operator can be eliminated everywhere except on the outside of the term.

A description with parameters are is called a functional relation, and the corresponding function assigns witnesses for each value of the parameters.

Definition by description is easy to overlook as a principle of reasoning in mathematics. It was first formulated correctly by Giuseppe Peano Pea97, §22], including the elimination theorem and the need to prove the properties of $\phi$ before introducing the $n . \phi n$. Numerous other accounts, most notably [RW13, *14], have failed to do this. It is nevertheless much more familiar than the abstract notion of sobriety in the monadic framework, so we have another example in which different axioms are appropriate in accounts of foundations and applications ( $c f . \S 3.8)$. 
9.12. The notion of general recursion weakens that of definition by description into a form that is more akin to computation. It involves a search or minimalisation operator $\mu$ such that, for any partial map $f: \mathbb{N} \rightarrow \mathbf{2}$,

$$
\mu(f)=n \quad \text { iff } \quad f(n)=1 \quad \text { but } \quad \forall m<n . f(m)=0 .
$$

If $f$ is total and $\exists n$. $(f n=1)$ then $\phi \equiv \lambda n .(f n=1) \wedge \forall m<n .(f m=0)$ is a description, for which $\mu(f)=$ the $n$. $\phi n$. However, we can use Rosolini's treatment of partial functions $(c f . \S 7.3)$, together with the construction of $\mathbb{N}_{\perp}$ in 8.11 , to define $\mu(f)$ for all $f: \mathbb{N} \rightarrow \mathbf{2}$.

First we need the fact that $\mathbf{2}_{\perp}$ is given by the closed subspace of $\Sigma \times \Sigma$ co-classified by $\lambda \sigma \tau . \sigma \wedge \tau$, and $\left(\mathbf{2}_{\perp}\right)^{\mathbb{N}}$ by that of $\Sigma^{\mathbb{N}} \times \Sigma^{\mathbb{N}}$ co-classified by $\lambda \phi \psi . \exists n$. $\phi n \wedge \psi n$.

The predicate $\theta n \equiv \phi(n) \wedge \forall m<n . \psi m$ satisfies the uniqueness requirement for a description, when we restrict $(\phi, \psi)$ to the closed subspace $\left(\mathbf{2}_{\perp}\right)^{\mathbb{N}} \subset \Sigma^{\mathbb{N}} \times \Sigma^{\mathbb{N}}$. Then, on the open subspace $P \subset\left(\mathbf{2}_{\perp}\right)^{\mathbb{N}}$ classified by $\exists n . \theta n$, i.e. the uniqueness condition, $\theta$ is a description, so defines a total map $P \rightarrow \mathbb{N}$ and a partial one $\left(\mathbf{2}_{\perp}\right)^{\mathbb{N}} \rightarrow \mathbb{N}$. The corresponding total map $\mu:\left(\mathbf{2}_{\perp}\right)^{\mathbb{N}} \rightarrow \mathbb{N}_{\perp}$ is the required minimalisation operator.

9.13. Given that the entire discipline of denotational semantics of programming languages is based on Scott continuity, it may come as a surprise that we can interpret general recursion without using it. This is because the (old-fashioned) search operator $\mu$ is only suitable for direct coding using base types such as $\mathbb{N}$ or $\mathbb{T}$.

For terms and parameters of these types, Scott continuity is a theorem, essentially the one of Henry Rice and Norman Shapiro Ric56. For any class $U$ of programs that is recursive enumerable using tests on their coding and pointwise values, a partial function is representable by a member of $U$ iff it contains some function with finite support that is also representable.

Looking at this type-theoretically, Scott continuity is provable for any expression with no free variables of type higher than $\Sigma^{\mathbb{N}}$. This is valid in practice for computation, because a program cannot proceed if there are any free variables (i.e. undefined values or sub-routines) at all. On the other hand, we use Scott continuity in the theory, not just to allow free variables, but because of the powerful conceptual analogy with general topology. ASD tightens this analogy by reformulating topology to fit Scott's theory of computation exactly.

We began this section by saying that overt discrete objects play the role of sets, but we have actually only used subquotients of $\mathbb{N}$. We need a further assumption in order to be able to work with abstract overt discrete spaces, i.e. general types for which $\exists$ and $=$ can be defined. Assuming Scott continuity, all overt discrete objects are subquotients of $\mathbb{N}$, and form an arithmetic universe (\$11.7).

\section{Underlying sets}

Since the work $[\mathrm{H}]$ on which this section is based was stalled, I need to look at it again to check certain assertions below.

In this section we consider an additional axiom that has the effect of turning the full subcategory of overt discrete spaces from a pretopos into an elementary topos. This allows us to compare ASD more closely with the textbook theories of topology that are based on set theory or toposes.

Since the new structure is not computable, it is a diversion from our main goal of formulating computable topology - I believe that the important features of general topology only depend on computable foundations. Nevertheless, it is methodologically important, because it shows which features of traditional topology we must sacrifice in the computable account.

The details behind this section may be found in $[\mathrm{H}]$. It offers one way in which the ideas of ASD might be extended beyond locally compact spaces, but there are currently no models apart 
from LKLoc with which to compare them. Nor is is clear whether this extension is compatible with the one that we discuss in $\$ 12$

10.1. Recall that the category BbkSp of Bourbaki spaces is related to the category of sets by the adjunctions in the diagram on the left, in which the downward functor yields the underlying set of points of a space and its adjoints equip any set with its finest and coarsest topologies.
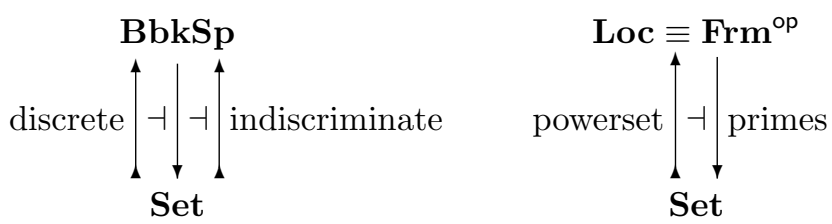

Considered as a frame, the powerset of a set provides its discrete topology, and therefore the left adjoint in the second diagram. Notwithstanding the fact that locales need not have enough primes $(\$ 4.2$ or points Joh82, $\S$ II 1.3] to make the functor faithful, the set of them gives the right adjoint. However, there is no such thing as the "indiscriminate" topology in locale theory, since by definition any locale has exactly the points that are determined by its open sets.

Topological spaces are axiomatised intrinsically in ASD, but could they possibly have "underlying sets" in a sense like this? As we explained in the previous section, overt discrete spaces play the role of sets, so the inclusion $\Delta: \mathcal{E} \subset \mathcal{S}$ of this full subcategory corresponds to the "discrete topology" functor above.

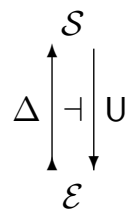

In this section, therefore, we assume that this inclusion has a right adjoint $\mathrm{U}$, called the underlying set functor. Since the objects of ASD, like locales, are all sober, there is no indiscriminate topology.

Recall our convention that $N$ and $M$ denote overt discrete objects.

10.2. What is the underlying set of the Sierpinski space $\Sigma$ or of the topology $\Sigma^{N}$ ? As we might expect from $\S \$ 7.5 \& 5.4$, they are respectively the subobject classifier $\Omega$ and powerset $\Omega^{N}$ of $N$ in $\mathcal{E}$. In particular, $\mathcal{E}$ is a topos.

The subcategory $\mathcal{E} \subset \mathcal{S}$ is closed under finite limits, including $\mathbf{1}$, products, equalisers, pullbacks and monos. Also, any mono in $\mathcal{E}$, being a map from an overt object to a discrete one, is an open inclusion $(8.10(\mathrm{~g})$. Hence any binary relation from $N$ to $M$ in $\mathcal{E}$, i.e. a mono $i: R \hookrightarrow N \times M$, is classified by $\phi: N \times M \rightarrow \Sigma$ in $\mathcal{S}$, as in the diagram on the left.
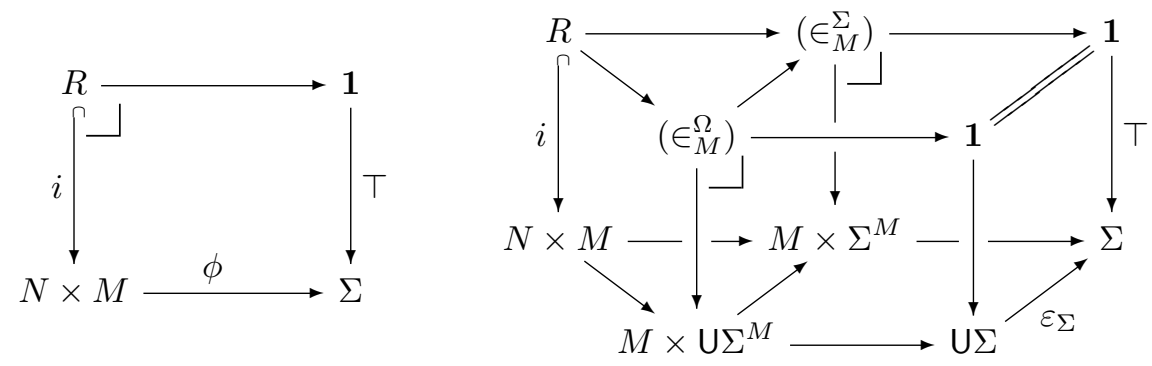

This square factorises into the two pullback squares at the back of the diagram on the right; in particular, $\phi=\mathrm{ev} \cdot \tilde{\phi}$, where $\tilde{\phi}: N \rightarrow \Sigma^{M}$ is the transpose of $\phi$. As the functor U preserves 
pullbacks, it takes the squares at the back of the diagram to those on the left and front (from $R$ to $\mathrm{U} \Sigma$ ). Hence

$$
\left(\in_{M}^{\Omega}\right) \equiv \mathrm{U}\left(\in_{M}^{\Sigma}\right) \hookrightarrow M \times \mathrm{U} \Sigma^{M}
$$

is the generic binary relation on $M$, as required to make $\mathcal{E}$ a topos.

The object $\mathrm{U}\left(\Sigma^{M}\right)$ has the universal property of the exponential $\Omega^{M}$ within $\mathcal{E}$, i.e. when we test it with maps $N \rightarrow \mathrm{U}\left(\Sigma^{M}\right)$ from overt discrete objects. However, this does not extend to general test objects in $\mathcal{S}$, and for this reason we prefer to write $\Omega M \equiv \mathrm{U}\left(\Sigma^{M}\right)$ instead of $\Omega^{M}$.

10.3. For reasons that we shall explain in $\S 10.8$, we say that an object $X \in \mathcal{S}$ has

(a) enough points if there is some epi $N \rightarrow X$, in which case $N \equiv \mathrm{U} X$ has this property; and

(b) enough opens if there is some equaliser $X \mapsto \Sigma^{N} \rightrightarrows \Sigma^{M}$, in which case we may choose $N \equiv \mathrm{U}\left(\Sigma^{X}\right)$ and $M \equiv \mathrm{U}\left(\Sigma^{3} X\right), c f . \$ 6.2$.

The converse to the result of the previous paragraph is that, if $\mathcal{E}$ is a topos then there is a partial right adjoint $\mathrm{U}$ that is defined on those objects that have enough opens.

10.4. We want to compare ASD with other theories of topology. The latter, i.e. the categories of frames, locales, locally compact locales and so on, can be constructed over any topos, such as $\mathcal{E}$, in essentially the same way as they are defined over sets. We can even pretend that our $\mathcal{E}$ is Set, so long as we understand "set theory" to mean the structure of an elementary topos. Our category $\mathcal{S}$ can also be treated in this way, because it is $\mathcal{E}$-enriched, i.e. its "homs" $\mathcal{S}(X, Y)$ can be given the structure of objects of $\mathcal{E}$.

The difference between ASD and the other theories is that

(a) the axiomatisation of the category $\mathcal{S}$ in ASD captures topological structure that is intrinsic to the category; whereas

(b) the structure in the other theories is imposed on (objects of) $\mathcal{E}$.

For example, $\leqslant(\$ 8.2)$ is intrinsic to $\Sigma^{X}$, but the arithmetical order $\leq$ is imposed on $\mathbb{N}(\$ 9.5)$. Notwithstanding its universal property, the structure on the object $\Omega$, including $\neg$ and $\Rightarrow$, is imposed in this sense $(\$ 10.7)$.

The objective is to show that the whole of our category $\mathcal{S}$ is equivalent to some category that is (re)constructed from objects of the subcategory $\mathcal{E}$.

10.5. We may compare $\mathcal{S}$ with categories such as Loc that are defined over $\mathcal{E}$. Consider the adjunctions amongst $\mathcal{S}^{\mathrm{op}}, \mathcal{S}$ and $\mathcal{E}$ that are given by the monadic framework $(\$ 6)$ and the underlying set hypothesis (10.1), as shown on the left:
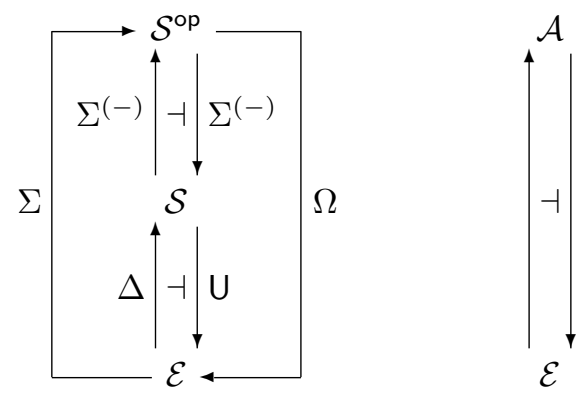

It is convenient to write $\Sigma \dashv \Omega$ for the composite functors. These induce a monad on $\mathcal{E}$, so let $\mathcal{A}$ be its category of Eilenberg-Moore algebras, and there is a comparison functor $\mathcal{S}^{\circ \mathrm{p}} \rightarrow \mathcal{A}$.

Even though $\mathcal{S}^{\text {op }} \rightleftarrows \mathcal{S}$ was assumed to be monadic, the composite need not be, i.e. the functor $\mathcal{S}^{\text {op }} \rightarrow \mathcal{A}$ need not be an equivalence. It is, however, full and faithful in the case where every object of $\mathcal{S}$ is definable in the monadic framework. This happens, for example, when $\mathcal{S}$ is the category of locally compact locales $(\$ 5.10)$. 
In general, this functor need only identify a full subcategory of $\mathcal{S}$ with one of $\mathcal{A}^{\mathrm{op}}$. The former consists of the spaces that have enough opens $(\$ 10.3)$, but further hypotheses are needed to fix the latter. In particular, we need Scott continuity to say that the monad on the topos $\mathcal{E}$ agrees with the one for Frm $(\S \$ 4.4 \& 11.8$ ).

10.6. The symbolic formulation of the underlying set functor is one of the clearest examples of translating an adjunction into a system of type-theoretic rules $(22.11$. For any type $X \in \mathcal{S}$, we have

(a) another type, the underlying set, $\mathrm{U} X$;

(b) as this is overt, an existential quantifier, $\left(\exists_{\cup X}\right): \Sigma^{U X} \rightarrow \Sigma$;

(c) as it is discrete, an equality, $(=\mathrm{U} X): \mathrm{U} X \times \mathrm{U} X \rightarrow \Sigma$;

(d) for any overt discrete context $\Gamma \in$ obE , a transposition (U-introduction)

$$
\frac{\Gamma \vdash a: X}{\Gamma \vdash \tau \cdot a: \mathrm{U} X}
$$

where the $\tau$ is accompanied by a dot because, like $\lambda$-abstraction, it is not an algebraic symbol but an operation on terms-in-context (whilst $\tau$ doesn't change the context $\Gamma$, it does depend on its belonging to $\mathcal{E})$;

(e) the counit (U-elimination), which is a function-symbol $x: \mathrm{U} X \vdash \varepsilon x: X$;

(f) satisfying the $\beta$ - and $\eta$-rules

$$
\Gamma \vdash \varepsilon(\tau . a)=a: X \quad \text { and } \quad x: \mathrm{U} X \vdash x=(\tau . \varepsilon x): \mathrm{U} X .
$$

In short, $\tau$. may be applied to any term so long as all of its free variables are of overt discrete type. In other words, it allows variation over a "combinatorial" structure but not a "geometrical" one.

10.7. The overt discrete object $\Omega$ carries imposed operations $(\curlywedge, \curlyvee, \leq)$ that make it a distributive lattice. These are just the U-images of the homologous intrinsic operations $(\wedge, \vee, \Rightarrow)$ on $\Sigma$, which remain nullary and binary on $\Omega$ because $U$ (being a right adjoint) preserves products. In particular, $(\leq): \Omega \times \Omega \rightarrow \Sigma$, cf. equality for discrete spaces in $\S \S 8.6 \& 9.3$.

But unlike $\Sigma, \Omega$ also has the imposed structure of a complete Heyting algebra, as does the set $\left(\mathcal{E}\right.$-object) $\Omega X \equiv \mathrm{U}\left(\Sigma^{X}\right)$ of opens of any space $X$, where $(\Rightarrow): \Omega X \times \Omega X \rightarrow \Omega X$ is defined by

$$
(\phi \Rightarrow \psi) \equiv \tau \cdot \lambda x: X . \exists \theta: \mathrm{U} \Sigma^{X} . \varepsilon \theta x \wedge(\phi \curlywedge \theta \leq \psi)
$$

and $\bigvee: \Sigma^{\Omega X} \rightarrow \Sigma^{X}$ by $\quad \bigvee F \equiv \lambda x: X . \exists \theta: \mathrm{U} \Sigma^{X} . F \theta \wedge(\varepsilon \theta) x$.

Moreover, for any map $f: X \rightarrow Y$ in $\mathcal{S}$, we have $f^{*} \dashv f_{*}$ with respect to the imposed order inherited from $\Omega$, where

$$
f^{*} \psi \equiv \Omega f \equiv \tau . \lambda x: X .(\varepsilon \psi)(f x)
$$

and

$$
f_{*} \phi \equiv \tau . \lambda y: Y . \exists \theta: \mathrm{U} \Sigma^{Y} .(\varepsilon \theta) y \wedge\left(f^{*} \theta \leq \phi\right) .
$$

10.8. Using this notation, we can ask whether the intrinsically continuous functions in the category $\mathcal{S}$ that we have axiomatised directly agree with the imposed structure of the categories of locales and Bourbaki spaces over the topos $\mathcal{E}$. If we are given an $\mathcal{S}$-morphism $g: X \rightarrow Y$, the maps $f \equiv \cup g$ and $H \equiv g^{*}$ make the two squares commute:
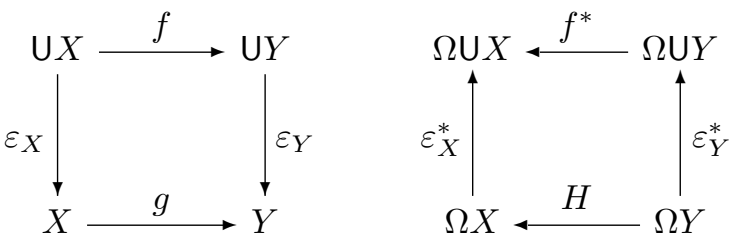
Conversely, if $X$ has enough points $\left(\varepsilon_{X}: \cup X \rightarrow X\right.$ is epi, 810.3$)$ then $\varepsilon_{X}^{*}: \Omega X \mapsto \Omega \cup X$ is split mono, so $\Omega X$ is a sublattice of the powerset of the underlying set of $X$, as in a Bourbaki space.

Then we may say that a function $f$ between the underlying sets is continuous in the imposed (Bourbaki) sense if there is a map $H$ that makes the square on the right commute. If $X$ has enough points and $Y$ has enough opens $(\sqrt[10.3]{)}$ then $f$ and $H$ arise from some unique $\mathcal{S}$-map $g$.

Once again, we have a lot of structure that looks like topology, even though we have said nothing to ensure that the category $\mathcal{A}$ of "algebras" in $\$ 10.5$ is actually that of frames over $\mathcal{E}$. The relevant condition is one of the formulations of Scott continuity that we consider in the next section (\$11.8). The result is that the full subcategory of spaces with enough points and enough opens is equivalent to the category of sober Bourbaki spaces over $\mathcal{E}$, and the whole category of Bourbaki spaces is equivalent to a full subcategory of the comma category $\mathcal{E} \downarrow \mathcal{S}$.

We shall not assume the underlying set axiom in the remainder of this paper.

\section{Scott continuity}

Although it is remarkable how much the theory already looks like traditional general topology without invoking it, we come to the point where we actually have to say that all functions are Scott continuous as an axiom.

Since this axiom completes the theory of locally compact spaces, we are moving into the territory of a user-oriented account $(c f . \sqrt[3]{3.8}$ ), but certain foundational issues remain. One of these is the question of which statement of the Scott continuity axiom we should adopt, since there are several candidates that arise from some of the applications of this idea.

11.1. Denotational semantics generalises the notion of recursion from minimalisation $(\mu, \$ 9.12)$ to fixed points of operators of higher types. Applying the operator $F$ repeatedly to $\perp$ gives an increasing sequence $\phi n \equiv F^{n} \perp$. Then $F$ fixes the join of such a sequence if we assume the rule

$$
\frac{\Gamma, n: \mathbb{N} \vdash \phi n: \Sigma^{U} \quad \Gamma, n: \mathbb{N} \vdash \phi n \leqslant \phi(n+1): \Sigma^{U} \quad \Gamma, \psi: \Sigma^{U} \vdash F \psi: \Sigma^{V}}{\Gamma \vdash F(\exists n . \phi n)=\exists n . F(\phi n): \Sigma^{V}}
$$

In this case, every space has and every map preserves joins of definable ascending chains with respect to the $\sqsubseteq$ order $(8.2$. Also, on any object that has $\perp$, every endomorphism has a least fixed point Tay91.

11.2. Turning to real analysis, a common use of the Heine-Borel theorem $(\$ 1.7)$ is to derive a uniform value that is valid throughout a compact space $K$ from local values at each point. For example, $b: \mathbb{R}$ is an upper bound on a function $f: K \rightarrow \mathbb{R}$ at $x$ if $\theta(x, b) \equiv(f x<b) \Leftrightarrow \top$. Then $\theta$ is monotone in the sense of taking the imposed arithmetical order $\leq$ on $\mathbb{R}$ to the intrinsic one $\Rightarrow$ on $\Sigma$ :

$$
\ldots, b \leq b^{\prime} \vdash \theta(x, b) \Longrightarrow \theta\left(x, b^{\prime}\right) .
$$

The $\delta>0$ that is used in the $\varepsilon-\delta$ definitions of continuity, differentiability, etc., is similar, but the defining property reverses the arithmetical order:

$$
\ldots, 0<\delta \leq \delta^{\prime} \vdash \theta(x, \delta) \Longleftarrow \theta\left(x, \delta^{\prime}\right) .
$$

In either case, by compactness, the quantifier $\forall_{K}$ satisfies

$$
\forall k . \exists b . \theta(k, b) \Longrightarrow \exists b . \forall k . \theta(k, b) .
$$

In [J], $\S \S 9-10]$ this is used to prove that any expression $\Gamma, x: \mathbb{R} \vdash a: \mathbb{R}$ is continuous in the $\varepsilon-\delta$-sense, and uniformly so on any compact domain. 
This principle, in which the second argument of $\theta$ ranges over $\mathbb{R}$, is equivalent to its analogue with $\mathbb{Q}$ instead. The latter is preferable foundationally ( $c f . \$ 3.8)$, as we would like to work with overt discrete spaces $(\$ 9$ ).

11.3. The indexing objects $\mathbb{N}$ and $\mathbb{Q}$ in these two properties have max operations, whilst we may form binary unions of finite open subcovers, so the common pattern is a directed join. In practice, we have a set (overt discrete space) $N$ of indices, equipped with an imposed semilattice operation $(+)$. Then we select an open subsemilattice of $N$ classified by $\alpha: \Sigma^{N}$. As in $\$ 5.8$, we use sub- and super-scripts to indicate co- and contra-variance with respect to this imposed order on $N$. Then a directed join, written

$$
\bigvee_{\left\{n: N \mid \alpha_{n}\right\}} \phi^{n} \equiv \exists n: N \cdot \alpha_{n} \wedge \phi^{n}
$$

involves a pair of families $\left(\alpha_{n}, \phi^{n}\right)$ such that

$$
\top \Leftrightarrow \exists n . \alpha_{n}, \quad \alpha_{n} \wedge \alpha_{m} \Leftrightarrow \alpha_{n+m} \quad \text { and } \quad \phi^{n} \vee \phi^{m} \leqslant \phi^{n+m} .
$$

Then we say that $F$ preserves this join if $F\left(\exists n . \alpha_{n} \wedge \phi^{n}\right)=\exists n . \alpha_{n} \wedge F \phi^{n}$.

To do this, + does not actually need to satisfy the equations for a semilattice, so the leading examples of $(N,+)$ are $(\mathbb{N}, \max ),(\mathbb{Q}, \max ),(\mathbb{Q}, \min ),(\mathrm{K}(M), \cup)$ and $(\operatorname{List}(M),+)$ for any overt discrete $M$.

11.4. In the first instance we shall be content with $N \equiv \mathbb{N}$, although it is equivalent but more useful to take $N \equiv \mathbb{T}$ instead, since List $(\mathbb{T}) \cong \mathbb{T}(\$ 9.6)$. For either of these $N$ s, we assert the $S$ cott continuity axiom in the form

$$
F: \Sigma^{\Sigma^{N}}, \phi: \Sigma^{N} \vdash F \phi \Longleftrightarrow \exists \ell: \operatorname{List} N . F(\lambda n . n \in \ell) \wedge \forall n \in \ell . \phi n .
$$

Consider in particular $\phi \equiv \lambda x . \sigma$ and $F \equiv \lambda \psi \cdot G(\psi 0)$, for any given $\sigma: \Sigma$ and $G: \Sigma^{\Sigma}$. Since it's decidable whether a list is empty or inhabited, $\exists \ell$ reduces to the disjunction of these two cases, so

$$
G \sigma \equiv F \phi \Longleftrightarrow G \perp \vee \sigma \wedge G \top \text {. }
$$

Hence the Phoa principle follows from that of Scott.

11.5. The Scott axiom for $N$ says that $\Sigma^{N}$ has a basis as a locally compact space, in the sense of $\S \$ 5.8$; , with

$$
\beta^{\ell} \equiv \lambda n . n \in \ell \quad \text { and } \quad A_{\ell} \equiv \lambda \phi . \forall n \in \ell . \phi n .
$$

This corresponds to the classical description of the Scott topology on $\mathcal{P}(N)$, whose open sets are $\mathcal{U} \equiv\{U \subset \mathbb{N} \mid \ell \subset U\}$ for all finite $\ell \subset \mathbb{N}$.

Another way of putting this is that $\Sigma^{\Sigma^{N}}$ is a retract of $\Sigma^{N}$, and it is not difficult to extend this to all objects that are definable using $\times$ and $\Sigma^{(-)}$. Hence any object that is definable in the monadic framework (\$6) is a $\Sigma$-split subspace of $\Sigma^{N}$ [G, $\left.\S 8\right]$.

G] goes on to show how any computably based locally compact Bourbaki space or locale $X$ may be "imported" into ASD. That is, suppose that $X$ has a basis $\left(K^{n}, V^{n}\right)$ indexed by $N$, such that the relation $(n \preccurlyeq m) \equiv\left(K^{n} \subset V^{m}\right)$ is recursively enumerable. Then this relation is a term $n, m: N \vdash(n \prec m): \Sigma$ satisfying certain properties [G] §11], from which we may define a nucleus on $\Sigma^{N}$ [G, §14], and so a type whose interpretation is $X$ [G, §17]. 
11.6. It is in this representation that we can show that the $\lambda$-calculus definitions of primes and nuclei $(\S ₫ 6.3 \& 6.6)$ are equivalent to more familiar lattice-theoretic ones [G, $\S 10]$.

We can also characterise certain classes of definable objects [G, §9]:

(a) any overt discrete space is the subquotient of $\mathbb{N}$ by some open partial equivalence relation;

(b) any compact overt discrete space is Kuratowski-finite (\$9.7); and

(c) any compact Hausdorff overt discrete space is finite, i.e. of the form $\{m \mid m<n\}$.

From (a) we may derive definition by description $(\$ 9.11)$, the free monoid $(\operatorname{List}(N), \S 9.7)$ and the Scott principle $(\$ 11.4)$ for all overt discrete $N$.

Beware, however, that all of these results apply just to objects that are definable in the monadic framework as we gave it in $\$ 6$. They will need to be proved again, or may cease to be valid, in a more general setting $(12$.

11.7. Even without assuming that $N$ is definable, we may use the intuitions gained from these results to construct List $(N)$ for any overt discrete $N$. The key idea is to encode any Kuratowskifinite $\ell \subset N$ by the pair of modal operators (88.8) that say that $\ell$ is overt and compact. Then $\operatorname{List}(N)$ is a $\Sigma$-split subspace of $\Sigma^{\Sigma^{N}} \times \Sigma^{\Sigma^{N}}$. It is obtained using a nucleus that is itself defined as a fixed point, using the basic Scott continuity axiom (\$11.1) and a tour de force of techniques in ASD and domain theory [E].

We deduce that the overt discrete objects form an arithmetic universe (99.7).

11.8. The general Scott continuity axiom in $\$ 11.4$ is only meaningful for general overt discrete $N$ that have $\operatorname{List}(N)$ or $\mathrm{K}(N)$. But we may obtain the latter from either

(a) the underlying set axiom ( $\$ 10$, in which case $\mathcal{P}(N) \equiv \Omega N$ exists, and $\mathrm{K}(N) \subset \Omega N$ is the semilattice generated by $N$ [Mik76, Appx 2]; or

(b) fixed points defined by joins of chains (11.1).

Armed with these, we are in a position to assert the Scott continuity axiom (\$11.4) for general overt discrete $N$.

In the setting of classical topology, the weaker axiom would only provide countable unions of open sets, whereas arbitrary cardinalities are required for the usual theory.

Similarly, in the context of our underlying set axiom, Scott continuity for general general overt discrete objects is exactly what is required to make the monad in $\$ 10.5$ agree with the one that defines the category of frames over $\mathcal{E}(\sqrt[4.4]{4}$. The full subcategory of spaces with enough points and enough opens then agrees with the category of sober Bourbaki spaces over $\mathcal{E}$.

11.9. Turning to the applications of general topology in elementary real analysis, this axiomatic setting (in fact, with just 11.1 is enough to construct the Dedekind reals using the nucleus that we mentioned in $\$ 5.12$ [I].

We may use this as an experimental test of the prototype axioms $(c f . \$ 3.2)$. The claim is that ASD is a theory of computable topology, and in particular that its terms $x: \mathbb{R} \vdash a: \mathbb{R}$ exactly capture the computably continuous functions $f: \mathbb{R} \rightarrow \mathbb{R}$. We already know that the interpretation of such a term in the model using locally compact locales $(5.10$ is continuous, whilst $[\mathbf{K}]$ gives some ideas of how to compute with our calculus.

So, is every classically continuous function $f: \mathbb{R} \rightarrow \mathbb{R}$ that can be computed by a program representable in ASD? First we need to agree on some notion of computability, but whatever yours is, I would hope that it could be adapted to provide a program $\pi(q, e, t)$ that takes three rational arguments and terminates iff $e<f(q)<t$. Using the denotational semantics of your programming language, interpreted in the domain theory of ASD, $\pi$ is a term of type $\Sigma^{\mathbb{Q}^{3}}$. From this we may derive a morphism $g: \mathbb{R} \rightarrow \mathbb{R}$ in ASD whose classical interpretation is the given function $f$. 
11.10. In foundational studies it has been long been customary to regard $\mathbb{R}$ as something constructed from $\mathbb{N}(c f . s 1.1(\mathrm{~b})]$, and indeed that is what we too have done $\mathbb{I}$. However, the importance of $\mathbb{R}$ throughout mathematics and science surely justifies introducing it as a base type, especially if we can formulate convincing axioms for it.

For these, we propose that

(a) it is an overt space, with $\exists_{\mathbb{R}}(8.10$;

(b) it is Hausdorff, with an inequality or apartness relation, $\neq(\$ 8.6)$;

(c) the closed interval $[0,1]$ is compact $(\S £ 1.7,5.7,5.12,8.7,11.2$;

(d) $\mathbb{R}$ has a total order $<$ with $(x \neq y) \Leftrightarrow(x<y) \vee(y<x)$;

(e) it is Dedekind complete, in a sense in which the two halves of a cut are open;

(f) it is a field, where $x^{-1}$ is defined iff $x \neq 0$;

(g) and Archimedean, i.e., for $x, y: \mathbb{R}$,

$$
y>0 \Rightarrow \exists n: \mathbb{Z} \cdot y(n-1)<x<y(n+1) .
$$

11.11. Dedekind completeness plays the same role for $\mathbb{R}$ that definition by description (\$9.11) does for $\mathbb{N}$, namely to recover numbers from the computation that we do in the arena of logic. Cuts and primes are inter-definable by

and

$$
(\delta, v) \equiv(\lambda d . P(\lambda x . d<x), \lambda u \cdot P(\lambda x . x<u))
$$$$
P \equiv \lambda \phi . \exists d<u . \delta d \wedge v u \wedge \forall x:[d, u] . \phi x,
$$

showing that Dedekind completeness cannot really be separated from the Heine-Borel theorem [I] $\S 14]$.

Once again, there is a system of logical rules, in which the definition of a Dedekind cut is the premise of the introduction rule for cut $(\delta, v)$, and the elimination rule represents any $a: \mathbb{R}$ by the cut

$$
\delta \equiv \lambda d . d<a \quad v \equiv \lambda u . a<u .
$$

Like focus and descriptions, Dedekind cut operations may be eliminated everywhere except on the outside of a term. Any cut sub-term has a smallest enclosing sub-term [cut $/ x]^{*} \sigma$ of type $\Sigma$ (typically $<, \neq$ or $>$ ), and then

$$
[\operatorname{cut}(\delta, v) / x]^{*} \sigma \Longleftrightarrow(\lambda x . \sigma) \operatorname{cut}(\delta, v) \Longleftrightarrow \exists d<u: \mathbb{Q} . \delta d \wedge v u \wedge \forall x:[d, u] . \sigma .
$$

11.12. The applications of this construction to elementary real analysis are begun in [J]. The principal objective of this paper is to solve equations defined by continuous functions $f: \mathbb{R} \rightarrow \mathbb{R}$, which makes novel use of overt subspaces. By way of an experimental test of our axioms for $\mathbb{R}$ ( $c f$. S 3.2 , it also proves the traditional characterisation of open subspaces of $\mathbb{R}$ as countable unions of disjoint open intervals, although the words "countable" and "interval" need some qualification. This result fails in real analysis without the Heine-Borel theorem, in particular in Bishop's theory BB85.

From here there should be no difficulty in developing a theory of differential and integral calculus on $\mathbb{R}^{\mathbf{n}}$. However, since the version of ASD that we have developed so far only gives an account of locally compact spaces, we cannot study Banach spaces and functional analysis until we have a much more general framework than the one in $\$ 6$. 


\section{Beyond local compactness}

This section requires more work, and in particular $\$ \$ 12.10 \mathrm{ff}$ should be replaced by a less technical conclusion that describes my current plans.

As we said in $\$ 3.7$, any generalisation of ASD must go way beyond the category of Bourbaki spaces. This section describes some investigations I am conducting while I write this story, so it therefore involves empirical tests, and carries significant risk of failure (\$3.9). Indeed, this is just what has happened to several previous attempts to find models.

12.1. Curiously, one way of extending the traditional categories to have all exponentials is to add new colimits, specifically quotients of equivalence relations. The new objects, as proposed by Dana Scott, are called equilogical spaces BBS04. Giuseppe Rosolini put them in a sheaf-like categorical framework [Ros00] that also includes Martin Hyland's earlier filter spaces Hyl79, and there also a cartesian closed extension for locales [Hec06]. Countably based equilogical spaces form a full subcategory of Bourbaki spaces MS03.

The equivalence relations in these constructions are not obliged to respect the topological structure in any way. For example, the four-point object that identifies $\perp$ of one copy of the Sierpiński space with $T$ of another has four open subspaces, but also a discrete two-element subspace, one of whose open subsubspaces cannot be extended to the whole space. Such objects owe more to set theory than to topology, but we can modify Scott's construction to eliminate them.

12.2. When we considered recursion in $\$ 9.8$, the main defect that we found in the monadic framework of 86 was not the lack of function spaces, but that of general equalisers. This was also the main problem in defining the compact subspace $K \subset X$ that corresponds to a modal operator $A: \Sigma^{2} X$ (\$8.9): we should have $K \equiv\{x: X \mid A \leqslant \lambda \phi . \phi x\}$, which is the equaliser of $\lambda \phi . A \phi$ and $\lambda \phi . A \phi \wedge \phi x$.

We adopted an $a d$ hoc solution to this in $\$ 9.10$ adding a second category $\mathcal{L}$ that has equalisers but not all powers of $\Sigma$. It does have some additional function-types, in particular $\mathbb{N}^{\mathbb{N}}$ and $\mathbb{R}^{\mathbb{R}}$, but not $\mathbb{N}^{\mathbb{N}^{\mathbb{N}}}$ or $\mathbb{R}^{\mathbb{R}^{\mathbb{R}}}$.

Plainly the natural step is to ask for both equalisers and powers of $\Sigma$. This simple hypothesis already leads to some interesting observations: a complicated subtype such as

$$
\left\{x: X \mid f x=g x: \Sigma^{\left\{y: Y \mid p y=q y: \Sigma^{Z}\right\}}\right\}
$$

invites re-writing as $\{x: X \mid \forall y: Y .(\forall z: Z$. pyz $=q y z) \Rightarrow(f x y=g x y)\}$. Here $\forall$ is a new kind of universal quantifier that, at first, we keep separate from $\forall$ for compactness ( $c f$. \$12.6). Also, whilst we have taken $\forall$ to bind more tightly than $\Rightarrow$, the scope of $\forall$ includes $\Rightarrow$ but is terminated by $\vdash$.

12.3. Let's put this a bit more precisely. As in $\$ 6$ the lattice structure of the object $\Sigma$ is not used in the fundamental construction, so it could perhaps be replaced by a different kind of algebra.

Consider any equaliser $i: E \nrightarrow X \rightrightarrows \Sigma^{Y}$ in a category $\mathcal{S}$ with all finite limits and powers of an object $\Sigma$. Then, for any $a: \Gamma \rightarrow X$ such that the composites $\Gamma \times Y \rightarrow X \times Y \rightrightarrows \Sigma$ are equal, 
there is a unique $b: \Gamma \rightarrow E$ such that $a=i \cdot b$.

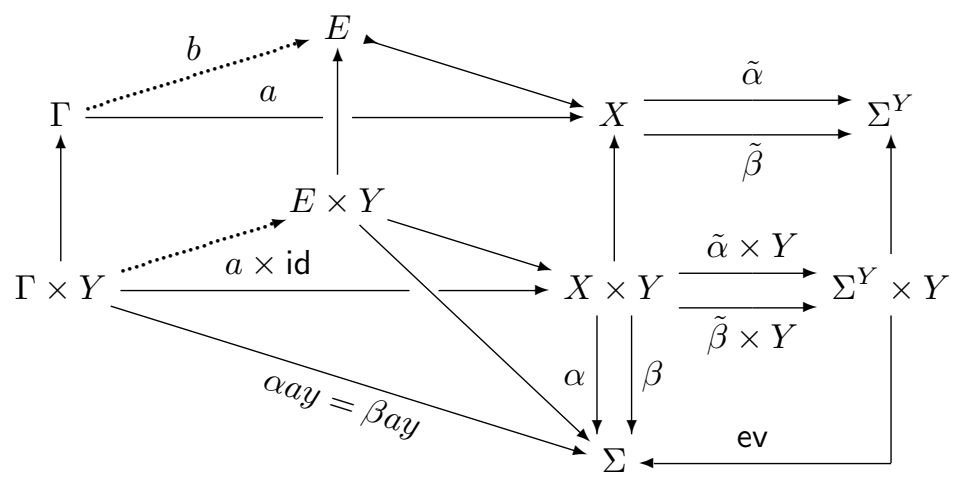

This property, which is called a partial product, is of interest because it can be stated entirely within any full subcategory $\mathcal{L} \subset \mathcal{S}$ that is closed under products and regular monos, by which we mean equalisers targeted at objects of $\mathcal{S}$ that need not belong to $\mathcal{L}$.

Following our method $(\$ 2.11)$, we can rewrite this categorical idea syntactically,

$$
\frac{x: X, y: Y, \mathfrak{p}(y) \vdash \alpha x y=\beta x y}{\overline{P: X \vdash \forall y: Y \cdot \mathfrak{p}(y) \Rightarrow \alpha x y=\beta x y}}
$$

where $\mathfrak{p}$ denotes a regular mono into $Y$. This generalises the diagram to allow the $Y$ there to be the result of a similar construction, and justifies the informal usage above. By a small modification to the diagram, it is easy to show that we may substitute for the free variable $x$ under this quantifier, $c f .22 .9$

Since taking the product of the targets of the equalisers provides the intersection of two regular monos, the logic also has conjunction, for which we write $\&$. Then we have

$$
\forall y \cdot \mathfrak{p}(y) \Rightarrow(\forall z \cdot \mathfrak{q}(z) \Rightarrow \alpha x y z=\beta x y z) \quad \dashv \quad \forall y z \cdot \mathfrak{p}(y) \& \mathfrak{q}(z) \Rightarrow \alpha x y z=\beta x y z,
$$

which also gives a meaning to $\forall y \cdot \mathfrak{p}(y) \Rightarrow \mathfrak{r}(x, y)$, so long as $\mathfrak{r}(x, y)$ is formed using $\forall \Rightarrow, \&, \top$ and equations.

12.4. Notice that, whilst it may range over any type,

$\forall$ must bind all of the variables that occur on the left hand side of $\Rightarrow$,

because we have not allowed the target $\Sigma^{Y}$ to be a dependent product.

One reason for avoiding dependent products is of course that they make things much more complicated, both categorically and syntactically. This restriction may therefore be loosened if we can construct examples of such products, or show that they are consistent. A situation in which we might reasonably expect to do that is indexing over $\mathbb{N}$, or more generally over any space that is either discrete or Hausdorff.

If you come from a type-theoretic background, you may be tempted to think, "this guy's just a categorist, we don't have any problem with dependent types", but I would point out that I have written a PhD thesis and a book on this topic Tay86, Tay99, mentioned in 82.6 . If you insert parameters into formulae without considering what they mean topologically, you are just being another logician who dictates foundations to mathematicians (\$1.1).

We cannot have dependent products in their full generality if we want to do topology rather than set theory ( $c f . \$ 2.9$ ). A category with unrestricted dependent products is called locally cartesian closed; since the exponentials are right adjoint to pullback, the latter preserves arbitrary colimits, in particular epis. 
For example, we write $\varpi$ for the ascending natural number domain, in which the topological order agrees with the arithmetical one, and Scott continuity creates a top element, so $0 \leqslant 1 \leqslant$ $\cdots \leqslant \infty$, as illustrated on the left:

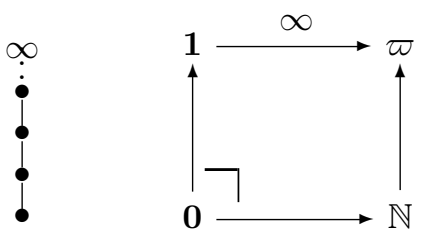

Then $\mathbb{N} \rightarrow \varpi$ is epi (in fact $\Sigma^{\varpi} \longmapsto \Sigma^{\mathbb{N}}$ is split mono), but not surjective, since $\infty$ has no inverse image, i.e. its pullback is the initial object.

Therefore, a category of "sober" spaces and Scott-continuous functions cannot be locally cartesian closed.

12.5. The new fragment with $\forall \Rightarrow, T$ and \& is called equideductive logic. Although it is rather feeble, it is interesting for a number of reasons. Besides being a pun on Scott's word, the name is justified because this is how we reason with proofs of equations in algebra.

Each judgement

$$
\begin{gathered}
x: X, y: Y, \ldots, a=b, c=d, \ldots \quad \vdash \quad e=f \\
\forall x: X . \forall y: Y \ldots a=b \& c=d \& \cdots \quad \Longrightarrow \quad e=f .
\end{gathered}
$$

becomes

Here, all of the variables are bound by $\forall$, but it's natural to relax this, using free variables of the new calculus in place of terms on the right of $\vdash$ that were previously metavariables in proof rules. The rule for $\forall \Rightarrow$ does, however, oblige us to bind all variables that occur in the equational hypotheses $a=b$, etc., but this seems to be natural. The horizontal line of a proof rule becomes another $\Rightarrow$, and the metavariables of the rule are bound by its $\forall$.

The bonus is that equideductive logic allows us to nest $\Rightarrow$ arbitrarily deeply. We may write induction as

$$
\forall n . \mathfrak{p}(0) \&(\forall m \cdot \mathfrak{p}(m) \Longrightarrow \mathfrak{p}(m+1)) \Longrightarrow \mathfrak{p}(n),
$$

in which $\mathfrak{p}(n) \equiv \forall x: X$. . . is the property to be proved. Note that, because of the variable binding rule, the variable $n$ can only occur in the equation at the right-hand end of $\mathfrak{p}(n)$. (Recall from $\$ 12.3$ how implications between predicates are reduced to a form with an equation on the right.)

If it is possible to relax this rule, allowing $m$ on the left of the innermost $\Rightarrow$ below, we may express course-of-values induction or well-foundedness of $<$ as

$$
\forall n .(\forall m . \quad(\forall k . k<m \Rightarrow \mathfrak{p}(k)) \Rightarrow \mathfrak{p}(m)) \Longrightarrow \mathfrak{p}(n) .
$$

Equideductive logic may be interpreted in the category of (sober) Bourbaki spaces, in the obvious way, namely by quantification over sets of points. However, having identified it categorically as a partial product, we may look for other semantic interpretations. These might include the category of locales or formal topology, but this is not clear.

In any model such as these, there are accidental containments between regular monos, represented syntactically by judgements $\mathfrak{p}(x) \vdash \mathfrak{q}(x)$ that need not follow from the axioms. In what follows, we shall understand the calculus in a purely syntactic way, without allowing particular concrete interpretations to impose accidental judgements over which we have no control. After that, we can add new axioms according to our own purposes (\$12.11). 
12.6. Combined with the lattice structure on the Sierpiński space, equideductive logic is also just what we need to form open, closed, compact and overt subspaces in ASD. Some of the following examples were first observed by Matija Pretnar.

As in 8.2 , we use $\Rightarrow$, $\leqslant$ and $\sqsubseteq$ for the order relation that arises from the lattice structure on $\Sigma$. The Gentzen rules (8.5) relate these orders to the new implication. Starting from these rules in the form

$$
\frac{x: X, \alpha x=\top \vdash \beta x=\top}{=x: X \vdash \alpha x \Rightarrow \beta x} \quad \text { and } \quad \frac{x: X, \beta x=\perp \vdash \alpha x=\perp}{x: X \vdash \alpha x \Rightarrow \beta x}
$$

the translation above yields

$$
(\forall x \cdot \alpha x=\top \Rightarrow \beta x=\top) \Leftrightarrow(\forall x \cdot \alpha x \Rightarrow \beta x) \Longleftrightarrow(\forall x \cdot \beta x=\perp \Rightarrow \alpha x=\perp) .
$$

Also recall that, via $\lambda$-abstraction, the bottom line of the rules is the the definition of $\alpha \leqslant \beta$.

This means that we may interpret any term $\alpha: \Sigma^{X}$ as an equideductive predicate $\forall x . \alpha x=\top$, the corresponding regular mono being an open inclusion $(c f . \$ 7.2)$. Then the lattice order relations $\Rightarrow, \leqslant$ and $\sqsubseteq$ are special cases of $\Rightarrow$. Similarly, the rules for $\wedge$ and $\vee$ say that $\wedge$ is a special case of \&:

$$
\alpha=\top \& \beta=\top \Longleftrightarrow \alpha \wedge \beta=\top \quad \text { and } \quad \alpha=\perp \& \beta=\perp \Longleftrightarrow \alpha \vee \beta=\perp .
$$

Recall that $=_{N}$ in a discrete space $N(\$ 8.6)$ was also a special case of general equality of terms,

$$
n=m \Longleftrightarrow\left(n={ }_{N} m\right)=\top, \quad \text { whilst } \quad h=k \Longleftrightarrow\left(h \neq_{H} k\right)=\perp
$$

in a Hausdorff space $H$. The quantifier $\forall$ in a compact space is related in a similar way to our more general quantifier $\forall, c f . \$ 5.7$

$$
(\forall x . \phi x=\top) \Longleftrightarrow(\forall x . \phi x)=\top, \quad \text { whilst } \quad(\forall x . \phi x=\perp) \Longleftrightarrow(\exists x . \phi x)=\perp
$$

in an overt space.

12.7. We can therefore just use the traditional symbols $=\wedge, \Rightarrow$ and $\forall$, without the variants $={ }_{N}, \&, \Rightarrow$ and $\forall$, but they generate two different logics:

(a) The inner one provides the terms of type $\Sigma$, which are observable properties or open subspaces; computably continuous functions are derived from these.

(b) The outer one is the logic of provable properties and general subspaces.

We may form $=, \neq, \forall$ or $\exists$ within the inner calculus so long as the relevant space is discrete, Hausdorff, compact or overt, as before. The other cases, including $\Rightarrow$, take us to the outer calculus.

By an idea that has seen many uses in logic, the formulae

$$
(\mathfrak{p} \curlyvee \mathfrak{q})(a) \equiv \forall \sigma \tau: \Sigma . \quad\left(\forall a^{\prime} \cdot \mathfrak{p}\left(a^{\prime}\right) \Rightarrow \sigma=\tau\right) \&\left(\forall a^{\prime \prime} \cdot \mathfrak{q}\left(a^{\prime \prime}\right) \Rightarrow \sigma=\tau\right) \Rightarrow \sigma=\tau
$$

and

$$
(\exists x \cdot \mathfrak{p})(a) \equiv \forall \sigma \tau . \quad\left(\forall a^{\prime} x \cdot \mathfrak{p}\left(x, a^{\prime}\right) \Rightarrow \sigma=\tau\right) \Rightarrow \sigma=\tau
$$

extend $\vee$ and $\exists$ from terms of type $\Sigma$ to general predicates. However, they only obey the distributive and Frobenius laws

$$
(\mathfrak{p} \curlyvee \mathfrak{q})(\vec{a}) \& \mathfrak{r}(\vec{b}) \dashv((\mathfrak{p} \& \mathfrak{r}) \curlyvee(\mathfrak{q} \& \mathfrak{r}))(\vec{a}, \vec{b}) \quad \text { and } \quad(\exists x \cdot \mathfrak{p})(\vec{a}) \& \mathfrak{r}(\vec{b}) \dashv(\quad \exists x \cdot \mathfrak{p} \& \mathfrak{r})(\vec{a}, \vec{b})
$$

when the variables $\vec{a}$ and $\vec{b}$ are disjoint. Also, unlike their powerful namesakes in intuitionistic logic, they do not allow us to recover a witness for $\exists$, or knowledge which disjunct (p or $\mathfrak{q}$ ) was true. 
12.8. Since we defined $\forall \Rightarrow$ in $\$ 12.3$ from equalisers of exponentials, we may form subspaces using comprehension, and terms belong to the subspace iff they satisfy the defining predicate. Unlike the nuclei in 6 , these may be manipulated in a way that is very similar to set theory. In particular, open, closed, compact and overt subspaces of $X$ are given by

$$
\{x \mid \theta x=\top\}, \quad\{x \mid \theta x=\perp\}, \quad\{x \mid \forall \theta . \square \theta \Rightarrow \theta x\} \quad \text { and } \quad\{x \mid \forall \theta . \theta x \Rightarrow \diamond \theta\} .
$$

We can use the arbitrarily nestable implications in equideductive logic to write more complicated definitions in general topology. For example, the overt subspace $I \subset X$ defined by a modal operator $\diamond$ is connected [J, §13] if

$$
\diamond \top \Leftrightarrow \top \text { and } \ldots, \phi, \psi: \Sigma^{X}, \phi \vee \psi=\top_{I} \vdash \diamond \phi \wedge \diamond \psi \Rightarrow \diamond(\phi \wedge \psi) \text {. }
$$

In the new notation, where $I \equiv\{x: X \mid \forall \theta . \theta x \Rightarrow \diamond \theta\}$, the second part of this becomes

$$
\forall \phi, \psi . \quad(\forall x .(\forall \theta \cdot \theta x \Rightarrow \diamond \theta) \Rightarrow \phi x \vee \psi x) \quad \Longrightarrow \quad(\diamond \phi \wedge \diamond \psi \Rightarrow \diamond(\phi \wedge \psi)) .
$$

Unfortunately, the variable-binding rule means that this formulation can only be used when $\diamond$ has no parameters, whereas these were an important feature of the treatment of the solution of equations in $[\mathrm{J}$. As we said in $\$ 12.4$, we would hope to allow discrete or Hausdorff parameters in a future generalisation. However, before you dismiss this as a defect of the calculus, you should think carefully whether it might be telling us something about the topology of the connected components of, for example, the zero-set of a function.

12.9. We are running ahead of ourselves: although we introduced equideductive logic as a necessary feature of a category with both exponentials and equalisers, we still have to show that it is sufficient. In fact, it provides exactly the abstract basis that is needed to construct (a version of) Scott's category of equilogical spaces. As in $\$ 6$, this construction is independent of the lattice structure on $\Sigma$.

We start with some urtypes that play the role of unions of algebraic lattices, closed under product and $\Sigma^{(-)}$. It is convenient to assume that they also admit stable disjoint sums, although these could be eliminated in what follows by using lists of urtypes instead. The urterms are generated from variables and certain combinators, for which we assert equations of type $\Sigma$ as equideductive axioms without $\Rightarrow$.

Then an equideductive space $X$ is a triple $(A, \mathfrak{p}, \mathfrak{q})$ where $A$ is an urtype, $\mathfrak{p}$ is a predicate in this logic on $\Sigma^{A}$, and $\mathfrak{q}$ one on $A$, for which

$$
\phi, \psi: \Sigma^{A}, \quad \mathfrak{p}(\phi), \quad(\forall a: A \cdot \mathfrak{q}(a) \Rightarrow \phi a=\psi a) \quad \vdash \quad \mathfrak{p}(\psi) .
$$

The left hand side of this is essentially the partial equivalence relation $\phi \sim \psi$ in Scott's construction. Hence $X$ is represented as a partial quotient of $\Sigma^{A}$; in particular $\mathbf{1}=(\mathbf{0}, \top, \top)$ and $\Sigma=(\mathbf{1}, \top, \top)$.

A function $M: X \equiv(A, \mathfrak{p}, \mathfrak{q}) \rightarrow Y \equiv(B, \mathfrak{r}, \mathfrak{s})$ is an equivalence class of urterms $M: \Sigma^{A} \rightarrow \Sigma^{B}$ such that

$$
\begin{gathered}
\phi: \Sigma^{A}, \quad \mathfrak{p}(\phi) \quad \vdash \quad \mathfrak{r}(M \phi) \\
\text { and } \quad \phi, \psi: \Sigma^{A}, \quad \mathfrak{p}(\phi), \quad \forall a \cdot \mathfrak{q}(a) \Rightarrow \phi a=\psi a \quad \\
\text { where } M_{1}=M_{2} \text { if } \quad \phi: \Sigma^{A}, b: B, \mathfrak{s}(b) \Rightarrow M \phi b=M \psi b,
\end{gathered}
$$

We have to make full use of the rules of the logic, including substitution under $\forall$ and the condition relating $\mathfrak{p}$ and $\mathfrak{q}$ for a space, just to prove that this is a category, $\mathcal{S}$. Then the product is

$$
(A, \mathfrak{p}, \mathfrak{q}) \times(B, \mathfrak{r}, \mathfrak{s}) \equiv\left(A+B, \mathfrak{p} \cdot \pi_{0} \& \mathfrak{r} \cdot \pi_{1},[\mathfrak{q}, \mathfrak{s}]\right),
$$


where $[\mathfrak{q}, \mathfrak{s}]$ is the predicate on $A+B$ given by $\mathfrak{q}$ on $A$ and $\mathfrak{s}$ on $B$. The equaliser

$$
(A, \mathfrak{t}, \mathfrak{q}) \stackrel{I}{\longrightarrow}(A, \mathfrak{p}, \mathfrak{q}) \underset{\mathrm{N}}{\stackrel{M}{\longrightarrow}}(B, \mathfrak{r}, \mathfrak{s})
$$

is given by

$$
\mathfrak{t}(\phi) \equiv \mathfrak{p}(\phi) \&(\forall b: B \cdot \mathfrak{s}(b) \Rightarrow M \phi b=N \phi b) .
$$

Finally, for $X \equiv(A, \mathfrak{p}, \mathfrak{q})$ the exponential $\Sigma^{X}$ is given by $\left(\Sigma^{A}, \mathfrak{q}^{\mathfrak{p}}, \mathfrak{p}\right)$, where

$$
\mathfrak{q}^{\mathfrak{p}}(F) \equiv \forall \phi, \psi: \Sigma^{A} \cdot \mathfrak{p}(\phi) \&(\forall a: A \cdot \mathfrak{q}(a) \Rightarrow \phi a=\psi a) \Longrightarrow F \phi=F \psi .
$$

Since the category has all finite limits and powers of $\Sigma$, it has an interpretation of equideductive logic and the examples that we have given. There is a syntactic criterion on $\mathfrak{p}$ and $\mathfrak{q}$ that characterises when $(A, \mathfrak{p}, \mathfrak{q})$ is definable using this structure, and we restrict the notion of equideductive space to these objects. General exponentials and other categorical operations may be derived from this.

If $\Sigma \equiv(\mathbf{1}, \top, \top)$ is a lattice and satisfies the rules in $\$ 12.6$ then it is a dominance, so we may reproduce (some of) the abstract topology in $\$ 8$

12.10. This is a very pretty picture, but it is misleading, as serious problems remain. As we have seen, we must take a lot of care over the variable-binding rule. It would be nice to weaken this, but its alternative may be more difficult for customers (i.e. mathematicians who are interested in the applications of topology, rather than logic itself) to use, in contrast to the clear topological motivation for only allowing $\forall$ over compact subspaces.

The similarity with set theory should also serve as a warning of a more fundamental issue for the new calculus. As it stands, it is neither an extension of the letter of ASD as set out in \$6, nor faithful to its spirit in ensuring that the objects that we construct have the right topology.

Take, for example, the "compact" subspace $K \equiv\{x: X \mid \forall \phi . \square \phi \Rightarrow \phi x\} \subset X$ that is defined by a modal operator $\square$. Is $K$ actually a compact space, with its own universal quantifier $\forall_{K}$ : $\Sigma^{K} \rightarrow \Sigma$ ? This is a problem that we could dodge in [J] because that studied a Hausdorff space $(\mathbb{R})$, where all compact subspaces are closed. Categorically, the inclusion $i: K \longmapsto X$ induces a map $\Sigma^{\Sigma^{i}}: \Sigma^{\Sigma^{K}} \rightarrow \Sigma^{\Sigma^{X}}$. For $K$ to be compact, we need to find $\Theta_{K}: \Sigma^{\Sigma^{K}}$ that this map takes to our given $\square: \Sigma^{\Sigma^{X}}$. The theory should then relate parametric modal operators to proper maps, showing that these obey the Beck-Chevalley condition 2.9 and descent properties.

Another way of seeing this issue is that $\Theta_{K}$ is a total function that it is convenient, both mathematically and computationally, to represent by the partial function $\square$. This is similar to a long-standing problem in recursion theory, concerning definable total maps of higher type such as $\mathbb{N}^{\mathbb{N}} \rightarrow \mathbb{N}$ or $\mathbb{R}^{\mathbb{R}} \rightarrow \mathbb{R}$. Amongst many authors, Hyland and Bauer studied it in their settings of filter and equilogical spaces Hyl79, BBS04].

Applications in functional analysis also have something to say about this. Typically, a space $X$ in this subject is not locally compact, so the space $C(X)$ of continuous functions $X \rightarrow \mathbb{R}$ cannot be the exponential $\mathbb{R}^{X}$ in the category of Bourbaki spaces. When I have asked analysts what "the right" topology on $C(X)$ might be, they have said that there are horses for courses. Since $X$ often carries either a metric (norm) or a measure (integration), there are various topologies on $C(X)$ defined from norms and semi-norms that are useful for different purposes.

Is there perhaps a fundamental topology on $Y^{X}$, for which the others are continuous additional structure? So long as we can at least agree to exclude the discrete one, the clear candidate is the point-open or weak $k^{*}$-topology, which is the weakest or coarsest one for which each ev $v_{x}: Y^{X} \rightarrow Y$ is continuous. This means that the topology on $Y^{X}$ is generated as an algebra by all of the $\lambda f . \phi(f x)$ for $\phi: \Sigma^{Y}$ and $x: X$.

Finally, we repeat that our category of equideductive spaces does not necessarily satisfy the sobriety and monadicity properties in $₫ 6$. 
12.11. There is an unambiguous, if rather tedious, procedure for investigating questions such as these.

Consider, for example, a modal operator $\square$ on an equideductive space $(A, \mathfrak{p}, \mathfrak{q})$. This is given by a certain urterm, of type $\Sigma^{3} A$. From this we may compute the subspace $K$ as an equaliser $(A, \mathfrak{t}, \mathfrak{q})$, and then also $\Sigma^{2} X$ and $\Sigma^{2} K$. The formulae for these are not very pretty, but the inclusions $i: K \longmapsto X$ and $\Sigma^{2} i$ are realised by the identity, so $\Theta_{K}$, if it exists, has the same urterm as $\square$.

The question is therefore whether this satisfies the equideductive properties that are required of a function $\Sigma^{K} \rightarrow \Sigma$. We write the hypotheses at the top of the page, the conclusion at the bottom and try to fill in the proof in between. In general, there need be no such proof using the abstract rules of the calculus, and nor need the property follow from the accidental judgements that hold in any particular model, such as Scott's original equilogical spaces.

This is where an abstract approach wins over a preconceived set-theoretic model, as we have left open the option to add more axioms. You can't fill in the necessary proof? No problem. Just add it as an axiom.

There is, of course, a price to pay for doing this. The resulting system may be inconsistent, in the sense of being able to prove undesirable theorems like $0=1$. Indeed, since we have already got a complete axiomatisation of computably based locally compact spaces, we don't want any additional equations to be provable for them. In terms of the construction of the category of equideductive spaces, we don't want any new equations between urterms without hypotheses.

For equideductive logic, its weakness is its strength, as we can easily apply some proof theory to it. In particular, using the analogy between propositions and types $(\sqrt{2.2})$, proofs in this fragment may be considered as typed $\lambda$-terms, and strongly normalise $(2.3)$. They also have a domain-theoretic interpretation [HP89, in which Scott continuity corresponds to the prooftheoretic observation that, in a proof whose conclusion is an equation, any $\forall$-formula that occurs as a hypothesis must be used by instantiation at finitely many terms. This suggests a technique for rearranging the proof of the hypotheses of an instance of an additional axioms into one of its conclusion, thereby demonstrate the conservativity of the new principle.

12.12. This may make a profitable architectural practice, but it's not good science. According to Karl Popper, we must lay down a theory that is coherent in itself, and put our head on the block with a prediction that is open to falsification. That is what ASD did for the Heine-Borel theorem (5.12). A new axiomatisation should state both its principles and its applications in its own language, not in some encoding, which is what the construction in 12.9 provides. For example, functional programming languages such as HASKELL HHPJW07] add expressiveness by developing the mathematical theory, not by taking short cuts in the underlying $\mathrm{C}$ implementation.

The principle for doing this was laid down by Galileo in his rejection of Aristotle: we assume that the universe beyond our own reach obeys the same laws as hold in familiar places. This has been applied many times in mathematics, in particular to successive generalisations of numbers and spaces: the complex numbers were introduced to solve quadratic and cubic equations, but otherwise their arithmetic agrees with the reals. In recursion theory, Scott continuity is a theorem for ground types, so we adopt it as an axiom for higher types (\$9.13), which are "beyond our reach" in the sense that actual computation cannot proceed when there are free variables.

The criterion by which we regard certain territory as familiar and believe that we have the correct laws for it is that we have several theories whose motivations come from different sources but which yield equivalent results. In the case of topology, this applies to locally compact spaces, because Bourbaki spaces and locales agree (give or take some Choice) [Joh82, Ch. VII] and another essentially equivalent version is given by [JKM01]. Whilst the old and new formulations of ASD are not yet compatible, they do provide further accounts of this topic that are more or less equivalent to the others. 
12.13. The more general spaces do not have plentiful compact subspaces, so what feature can they have in common with locally compact ones? For this we have to examine the construction in $\$ 12.9$ more carefully. It is by no means the free extension to a category with all finite limits and exponentials. It has an exactness property, relating limits and colimits, that marshals the otherwise chaotic information about successive equalisers and exponentials into just two predicates on an urtype. On the full subcategory $\mathcal{L} \subset \mathcal{S}$ consisting of the $(A, \mathfrak{p}, \top)$, all objects are sober and $\Sigma^{2}$ preserves regular monos. This property is similar to but not the same as monadicity in ASD (\$6).

However, this property does not extend to the whole of the category of equideductive spaces. Following the Galilean principle, maybe we should add this, rather than ad hoc properties from potential applications, to the axioms of equideductive logic.

But it is not clear whether this is possible. Consider, for example, Baire space, $\mathbb{N}^{\mathbb{N}}$. This has a regular mono $X \equiv \mathbb{N}^{\mathbb{N}} \longmapsto \Sigma^{\mathbb{N} \times \mathbb{N}} \equiv A$ that cannot be $\Sigma$-split since $\mathbb{N}^{\mathbb{N}}$ is not locally compact. Hence $\mathrm{ev}_{X}$ classifies an open subspace of $\Sigma^{X} \times X$ that does not extend to one of $\Sigma^{X} \times A$. However, applying our proposed exactness property to $i: \Sigma^{X} \times X \longmapsto \Sigma^{X} \times A$ would make $\Sigma^{i}$ a categorical epi.

On the other hand, there are grounds for believing that the proposal is nevertheless consistent. Whilst rejecting its naïve applications, we have on several occasions exploited the analogy with set theory. In 89.3 we showed that the ASD axioms yield a topos if we require all objects to be overt discrete, in which case all monos are $\Sigma$-split. This situation is also a (degenerate) case case of $\$ 12.9$. The difference is that

(a) in set theory, all objects are (compact,) overt and discrete, which as we saw in $\$ 10$, is logically extremely powerful; whilst

(b) topology obeys Scott continuity, which, in its most general form (\$11.4), is just an $\Rightarrow$-free equation in equideductive logic.

12.14. If the example that we gave does not actually threaten the consistency of the proposal, it means that some of the generalised spaces would be even more lacking in points than locales are.

What more powerful conceptual tools might we gain from this? Our pointless extended calculus might be a good setting in which to study measure theory or distributions, since integration is closely analogous to the modal logic that we have used for compact and overt subspaces.

Such applications in analysis are one of the three ways in which I envisage developing ASD in the coming years. However, further work is required in the foundations of the subject to justify this, and then the structure that we have discussed in this paper needs to be reformulated, using the new foundations, in a textbook style. Finally, the claim that the theory is amenable to computational interpretation $(\$ 2.3)$ needs to be put into practice.

Acknowledgements: I would like to thank Andrej Bauer, David Corfield, Anders Kock, Fred Linton, Gabor Lukasz, Andy Pitts, Pino Rosolini, Giovanni Sommaruga, Hayo Thielecke and Graham White for their very helpful comments on earlier drafts of this paper.

\section{References}

[AGV64] Michael Artin, Alexander Grothendieck, and Jean-Louis Verdier, editors. Séminaire de Géometrie Algébrique, IV: Théorie des Topos, number 269-270 in Lecture Notes in Mathematics. Springer-Verlag, 1964. Second edition, 1972.

[App92] Andrew Appel. Compiling with Continuations. Cambridge University Press, 1992.

[Bau08] Andrej Bauer. Efficient computation with the Dedekind reals. In Vasco Brattka, Ruth Dillhage, Tanja Grubba, and Angela Klutsch, editors, Computability and Complexity in Analysis, 2008. 
[BB85] Errett Bishop and Douglas Bridges. Constructive Analysis. Number 279 in Grundlehren der mathematischen Wissenschaften. Springer-Verlag, 1985.

[BBS04] Andrej Bauer, Lars Birkedal, and Dana Scott. Equilogical spaces. Theoretical Computer Science, 315:35-59, 2004.

[BGvO71] Michael Barr, Pierre Grillet, and Donovan van Osdol, editors. Exact Categories and Categories of Sheaves. Number 236 in Lecture Notes in Mathematics. Springer-Verlag, 1971.

[Bou66] Nicolas Bourbaki. Topologie Générale. Hermann, 1966. English translation, "General Topology", distrubuted by Springer-Verlag, 1989.

[BR87] Douglas Bridges and Fred Richman. Varieties of Constructive Mathematics. Number 97 in LMS Lecture Notes. Cambridge University Press, 1987.

[Bro64] Ronald Brown. Function spaces and product topologies. Quarterly Journal of Mathematics, 15:238-250, 1964.

[CH06] Felice Cardone and Roger Hindley. History of lambda-calculus and combinatory logic. Handbook of the History of Logic, 5, 2006.

[Cle87] John Cleary. Logical arithmetic. Future Computing Systems, 2:125-149, 1987.

[CLW93] Aurelio Carboni, Steve Lack, and Robert Walters. Introduction to extensive and distributive categories. Journal of Pure and Applied Algebra, 84:145-158, 1993.

[Coc93] Robin Cockett. Introduction to distributive categories. Mathematical Structures in Computer Science, 3:277-307, 1993.

[Cor03] David Corfield. Towards a Philosophy of Real Mathematics. Cambridge University Press, 2003.

[CR36] Alonzo Church and Barkley Rosser. Some properties of conversion. Trans. AMS, 39:472-482, 1936.

[EM65] Samuel Eilenberg and John Moore. Adjoint functors and triples. Illinois Journal of Mathematics, 9:381-98, 1965.

[FG87] John Fauvel and Jeremy Gray. The History of Mathematics, a Reader. Macmillan and the Open University, 1987.

[FH79] Michael Fourman and Martin Hyland. Sheaf models for analysis. In Applications of Sheaves, number 753 in Lecture Notes in Mathematics, pages 280-301. Springer-Verlag, 1979.

[Fox45] Ralph Fox. On topologies for function-spaces. Bulletin of the American Mathematical Society, 51:429-32, 1945.

[Gen35] Gerhard Gentzen. Untersuchungen über das Logische Schliessen. Mathematische Zeitschrift, 39:176-210 and 405-431, 1935. English translation in Gen69, pages 68-131.

[Gen69] Gerhard Gentzen. The Collected Papers of Gerhard Gentzen. Studies in Logic and the Foundations of Mathematics. North-Holland, 1969. Edited by M. E. Szabo.

[GHK $\left.{ }^{+} 80\right]$ Gerhard Gierz, Karl Heinrich Hoffmann, Klaus Keimel, Jimmie Lawson, Michael Mislove, and Dana Scott. A Compendium of Continuous Lattices. Springer-Verlag, 1980. Second edition, Continuous Lattices and Domains, published by Cambridge University Press, 2003.

[Gir72] Jean Giraud. Classifying topos. In Bill Lawvere, editor, Toposes, Algebraic Geometry, and Logic, number 274 in Lecture Notes in Mathematics, pages 43-56. Springer-Verlag, 1972.

[Gir87] Jean-Yves Girard. Linear logic. Theoretical Computer Science, 50:1-102, 1987.

[Göd31] Kurt Gödel. Über formal unentscheidbare Sätze der Principia Mathematica und verwandter Systeme I. Monatshefte für Mathematik und Physik, 38:173-198, 1931. English translations, "On Formally Undecidable Propositions of 'Principia Mathematica' and Related Systems" published by Oliver and Boyd, 1962 and Dover, 1992; also in "From Frege to Gödel: a 
Source Book in Mathematical Logic, 1879-1931", ed. Jan van Heijenoort, Harvard University Press, 1967.

[Har77] Robin Hartshorne. Algebraic Geometry. Number 52 in Graduate Texts in Mathematics. Springer-Verlag, 1977.

[Hec06] Reinhold Heckmann. A cartesian closed extension of the category of locales. Mathematical Structures in Computer Science, 16(02):231-253, 2006.

[HHPJW07] Paul Hudak, John Hughes, Simon Peyton-Jones, and Philip Wadler. A history of Haskell: Being lazy with class. In History of Programming Languages. ACM Press, 2007.

[HM81] Karl Hofmann and Michael Mislove. Local compactness and continuous lattices. In Bernhard Banaschewski and Rudolf-Eberhard Hoffmann, editors, Continuous Lattices, volume 871 of Springer Lecture Notes in Mathematics, pages 209-248, 1981.

[How80] William Howard. The formulae-as-types notion of construction. In Haskell Curry, Jonathan Seldin, and Roger Hindley, editors, To H.B. Curry: Essays on Combinatory Logic, Lambda Calculus and Formalism, pages 479-490. Academic Press, 1980.

[HP89] Martin Hyland and Andrew Pitts. The theory of constructions: Categorical semantics and topos-theoretic models. In John Gray and Andre Scedrov, editors, Proceedings of the Boulder Conference on Categories in Computer Science and Logic, volume 92 of Contemporary Mathematics, pages 137-199, Providence, Rhode Island, 1989. American Mathematical Society.

[Hud89] Paul Hudak. Conception, evolution, and application of functional programming languages. ACM Computing Surveys, 21(3), 1989.

[Hyl79] Martin Hyland. Filter spaces and continuous functionals. Annals of Mathematical Logic, 16:101-143, 1979.

[Hyl91] Martin Hyland. First steps in synthetic domain theory. In Aurelio Carboni, Maria-Cristina Pedicchio, and Giuseppe Rosolini, editors, Proceedings of the 1990 Como Category Conference, volume 1488 of Lecture Notes in Mathematics, pages 131-156. Springer-Verlag, 1991.

[Isb86] John Isbell. General function spaces, products and continuous lattices. Mathematical Proceedings of the Cambridge Philosophical Society, 100:193-205, 1986.

[JKM01] Achim Jung, Mathias Kegelmann, and Andrew Moshier. Stably compact spaces and closed relations. Electronic Notes in Theoretical Computer Science, 45, 2001.

[Joh82] Peter Johnstone. Stone Spaces. Number 3 in Cambridge Studies in Advanced Mathematics. Cambridge University Press, 1982.

[Joh84] Peter Johnstone. Open locales and exponentiation. Contemporary Mathematics, 30:84-116, 1984.

[JT84] André Joyal and Myles Tierney. An extension of the Galois theory of Grothendieck. Memoirs of the American Mathematical Society, 51(309), 1984.

[Koc05] Anders Kock. Synthetic Differential Geometry. Number 333 in London Mathematical Society Lecture Note Series. Cambridge University Press, second edition, 2005.

[Kuh62] Thomas S Kuhn. The Structure of Scientific Revolutions. University of Chicago Press, 1962.

[Kur20] Kazimierz Kuratowski. Sur la notion d'ensemble fini. Fundamenta Mathematicae, 1:130-1, 1920.

[Lak63] Imre Lakatos. Proofs and refutations: the logic of mathematical discovery. British Journal for the Philosophy of Science, 14:1-25, 1963. Edited by John Worrall and Elie Zahar, Cambridge University Press, 1976.

[Law69] Bill Lawvere. Adjointness in foundations. Dialectica, 23:281-296, 1969. Reprinted with commentary in Theory and Applications of Categories Reprints, 16:1-16, 2006. 
[Lin69] Fred Linton. An outline of functorial semantics. In Beno Eckmann, editor, Seminar on Triples and Categorical Homology Theory, number 80 in Lecture Notes in Mathematics, pages 7-52. Springer-Verlag, 1969.

[Mai03] Marie Emilia Maietti. Joyal's arithmetic universes via type theory. Electronic Notes in Theoretical Computer Science, 69, 2003.

[Mai05] Marie Emilia Maietti. Reflection into models of finite decidable FP-sketches in an arithmetic universe. Electronic Notes in Theoretical Computer Science, 416, 2005.

[McC78] John McCarthy. History of LISP. History of programming languages, pages 173-185, 1978.

[McL90] Colin McLarty. The uses and abuses of the history of topos theory. British Journal for the Philosophy of Science, 41(3):351-375, 1990.

[Mik76] Christian Mikkelsen. Lattice-theoretic and Logical Aspects of Elementary Topoi. PhD thesis, Århus Universitet, 1976. Various publications, number 25.

[ML63] Saunders Mac Lane. Natural associativity and commutativity. Rice University Studies, 49:28-46, 1963.

[ML88] Saunders Mac Lane. Categories and concepts in perspective. In Peter Duren, Richard A. Askey, and Uta C. Merzbach, editors, A Century of Mathematics in America, volume 1, pages 323-365. American Mathematical Society, 1988. Addendum in volume 3, pages 439-441.

[Moo66] Ramon Moore. Interval Analysis. Prentice Hall, 1966.

[MS03] Matías Menni and Alex Simpson. Topological and limit-space subcategories of countably-based equilogical spaces. Mathematical Structures in Computer Science, 12(6):739-770, 2003.

[Par74] Robert Paré. Colimits in topoi. Bulletin of the American Mathematical Society, 80(3):556-561, May 1974.

[Pea97] Giuseppe Peano. Studii di logica matematica. Atti della Reale Accademia di Torino, 32:565-583, 1897. Reprinted in Peano, Opere Scelte, Cremonese, 1953, vol. 2, pp. 201-217, and (in English) in Hubert Kennedy, Selected Works of Giuseppe Peano, Toronto University Press, 1973, pp 190-205.

[Pho90] Wesley Phoa. Domain Theory in Realizability Toposes. PhD thesis, Cambridge, 1990. http://www.lfcs.informatics.ed.ac.uk/reports/91/ECS-LFCS-91-171 University of Edinburgh Dept. of Computer Science report CST-82-91 and ECS-LFCS-91-171.

[Pit00] Andrew Pitts. Categorical logic. In Handbook of Logic in Computer Science, volume 5, pages 39-128. Oxford University Press, 2000.

[Ric56] Henry Rice. On completely recursively enumerable classes and their key arrays. Journal of Symbolic Logic, 21:304-8, 1956.

[Ros86] Giuseppe Rosolini. Continuity and Effectiveness in Topoi. D. phil. thesis, University of Oxford, 1986.

[Ros00] Giuseppe Rosolini. Equilogical spaces and filter spaces. Rendiconti del Circolo Matematico di Palermo, Supplemento, 64:157-175, 2000.

[RW13] Bertrand Russell and Alfred North Whitehead. Principia Mathematica. Cambridge University Press, 1910-13.

[Sco72] Dana Scott. Continuous lattices. In Bill Lawvere, editor, Toposes, Algebraic Geometry and Logic, number 274 in Lecture Notes in Mathematics, pages 97-136. Springer-Verlag, Berlin, 1972.

[Sco93] Dana Scott. A type-theoretical alternative to ISWIM, CUCH, OWHY. Theoretical Computer Science, 121:422-440, 1993. Written in 1969. 
[Sel02] Jonathan Seldin. Curry's anticipation of the types used in programming languages. Proceedings of the Canadian Society for History and Philosophy of Mathematics, 15:148-163, 2002.

[Sim78] Harold Simmons. The lattice-theoretic part of topological separation properties. Proceedings of the Edinburgh Mathematical Society (2), 21:41-8, 1978.

[Smo06] Lee Smolin. The Trouble with Physics. Houghton Mifflin, 2006. Republished by Penguin, 2008.

[Spi07] Bas Spitters. Locatedness and overt sublocales. www.cs.kun.nl/ spitters/located.pdf, 2007.

[Ste67] Norman Steenrod. A convenient category of topological spaces. Michigan Mathematics Journal, 14:133-152, 1967.

[Sto37] Marshall Stone. Applications of the theory of Boolean rings to general topology. Transactions of the American Mathematical Society, 41:375-481, 1937.

[Sto38] Marshall Stone. The representation of Boolean algebras. Bulletin of the American Mathematical Society, 44:807-816, 1938.

[Tar35] Alfred Tarski. Zur Grundlegung der Boole'schen algebra. Fundamenta Mathematicae, 24:177-198, 1935.

[Tay86] Paul Taylor. Recursive Domains, Indexed category Theory and Polymorphism. PhD thesis, Cambridge University, 1986.

[Tay91] Paul Taylor. The fixed point property in synthetic domain theory. In Gilles Kahn, editor, Logic in Computer Science 6, pages 152-160. IEEE, 1991.

[Tay99] Paul Taylor. Practical Foundations of Mathematics. Number 59 in Cambridge Studies in Advanced Mathematics. Cambridge University Press, 1999.

[Thi97] Hayo Thielecke. Categorical Structure of Continuation Passing Style. PhD thesis, University of Edinburgh, 1997. Also available as technical report ECS-LFCS-97-376.

[Tur35] Alan Turing. On computable numbers with an application to the Entscheidungsproblem. Proceedings of the London Mathematical Society (2), 42:230-265, 1935.

[Ver94] Japie Vermeulen. Proper maps of locales. Journal of Pure and Applied Algebra, 92:79-107, 1994.

[Wil70] Peter Wilker. Adjoint product and hom functors in general topology. Pacific Journal of Mathematics, 34:269-283, 1970.

The papers on abstract Stone duality may be obtained from

\section{www.Paul Taylor.EU/ASD}

[O] Paul Taylor, Foundations for Computable Topology. in Giovanni Sommaruga (ed.), Foundational Theories of Mathematics, Kluwer 2009.

[A] Paul Taylor, Sober spaces and continuations. Theory and Applications of Categories, 10(12):248-299, 2002.

[B] Paul Taylor, Subspaces in abstract Stone duality. Theory and Applications of Categories, 10(13):300-366, 2002.

[C] Paul Taylor, Geometric and higher order logic using abstract Stone duality. Theory and Applications of Categories, 7(15):284-338, 2000.

[D] Paul Taylor, Non-Artin gluing in recursion theory and lifting in abstract Stone duality. 2000.

[E] Paul Taylor, Inside every model of Abstract Stone Duality lies an Arithmetic Universe. Electronic Notes in Theoretical Computer Science 122 (2005) 247-296, Elsevier.

[F] Paul Taylor, Scott domains in abstract Stone duality. March 2002. 
[G-] Paul Taylor, Local compactness and the Baire category theorem in abstract Stone duality. Electronic Notes in Theoretical Computer Science 69, Elsevier, 2003.

[G] Paul Taylor, Computably based locally compact spaces. Logical Methods in Computer Science, 2 (2006) 1-70.

[H-] Paul Taylor, An elementary theory of the category of locally compact locales. APPSEM Workshop, Nottingham, March 2003.

[H] Paul Taylor, An elementary theory of various categories of spaces and locales. November 2004.

[I] Andrej Bauer and Paul Taylor, The Dedekind reals in abstract Stone duality. Computability and Complexity in Analysis, Kyoto, August 2005.

$[\mathrm{J}]$ Paul Taylor, A $\lambda$-calculus for real analysis. Computability and Complexity in Analysis, Kyoto, August 2005.

[K] Paul Taylor, Interval analysis without intervals. February 2006.

[L] Paul Taylor, Tychonov's theorem in abstract Stone duality. September 2004. 